Portland State University

PDXScholar

$11-23-2021$

\title{
"It's Not by Accident": Examining Leadership Efforts to Disrupt Oregon's Segregated K-12 Education System
}

Michael Eric Salitore

Portland State University

Follow this and additional works at: https://pdxscholar.library.pdx.edu/open_access_etds

Part of the Special Education and Teaching Commons Let us know how access to this document benefits you.

\section{Recommended Citation}

Salitore, Michael Eric, "'It's Not by Accident": Examining Leadership Efforts to Disrupt Oregon's Segregated K-12 Education System" (2021). Dissertations and Theses. Paper 5840.

https://doi.org/10.15760/etd.7711

This Dissertation is brought to you for free and open access. It has been accepted for inclusion in Dissertations and Theses by an authorized administrator of PDXScholar. Please contact us if we can make this document more accessible: pdxscholar@pdx.edu. 
"It's Not by Accident": Examining Leadership Efforts to Disrupt Oregon's

Segregated K-12 Education System

by

Michael Eric Salitore

A dissertation submitted in partial fulfillment of the requirements for the degree of

\author{
Doctor of Education \\ in \\ Educational Leadership: Special and Counselor Education
}

\author{
Dissertation Committee: \\ Chris Borgmeier, Chair \\ Molly Siuty \\ Ruth Falco \\ Pat Burk \\ Christina Gildersleeve-Neumann
}

Portland State University

2021 
(C) 2021 Michael Eric Salitore 


\begin{abstract}
The American education system systematically and persistently excludes students from the general education setting based on (dis)ability. Disproportionate segregation of students with Intellectual Disability (ID) is a form of prejudice that is acceptable today and allowable by current laws. Fully segregated education settings for students with ID are harmful to students with disabilities, to their neurotypical peers, and to civilized society as a whole (Andresen \& Nord, 2020; Ballard \& Dymond, 2018; C. Cole et al., 2004; S. M. Cole et al., 2020; Cosier et al., 2013; Dessemontet et al., 2012; Kleinert et al., 2015; Ryndak et al., 2010; Soukup et al., 2007; Ryndak et al., 1999; Vinodrao, 2016). For many students with ID, ableist systems, deficit thinking, and special education rules allow for segregated placements to persist, impacting their pathway to accessing the general education curriculum (Agran et al., 2020; Brock, 2018; Morningstar et al., 2017).

Improving inclusive practices as a research-based practice for students with disabilities (Jackson et al., 2008) can lead to a decrease in segregated education, increase access to the general curriculum, and impact long-term outcomes for students with ID. This study examines the problem of segregated educational settings and how leaders in three Oregon school districts improve inclusive education by employing a multiple-case study. This study finds that aligned leadership, establishing a culture of inclusion, and intentional structures of support indirectly address ableism and influence
\end{abstract}


the technical and adaptive shifts necessary to improve inclusive education for students with ID. 


\section{Dedication}

This work is dedicated to the memory of my late Father-in-Law, Edward G. Miller (1942-2016), who modeled with his life, what is most important. 


\section{Acknowledgements}

Throughout the writing of this dissertation, I have received a great deal of support and assistance. I would first like to thank my chair, Dr. Borgmeier, whose expertise and passion for high-quality public education for all students supported this project. You pushed my thinking to deeper levels of understanding. I would like to thank my committee members. In particular, Dr. Molly Siuty, whose expertise in qualitative research and analysis supported this project to surface the voice of the interview participants. In addition, Dr. Pat Burk, Dr. Ruth Falco, and Dr. Christina GildersleeveNewman for your feedback, encouragement, support, expertise, and experience leading equitable practices for students with disabilities. Your guidance helped shape my thinking and produced (hopefully) a valuable contribution to the scholarship of improving public education.

I wish to acknowledge Dr. Eric Wells, Roberta Dunn and Dr. Jennifer Spencerliams, all of whom are colleagues in the work and share the same core values of the importance of inclusive education for students experiencing disability. Perhaps most importantly, I wish to acknowledge the leaders who participated in this research project. To highlight your leadership practices focused on equitable outcomes for all students is the focus of this work and the center of my learning. If I was able to highlight the passion, expertise, and bold leadership you live every day, my goal is accomplished. I acknowledge Ramona Shockley. Ramona is currently a high school student, and a student for a time at the middle school in my school district. She has been my 
"anchor" student for many years, and perhaps my greatest teacher, modeling the lesson about what is possible when the adults are willing to be open and listen. Here is to you, Ramona, for your courage and heart!

Finally, I wish to thank my wife, Monica, and my children, Danica and Gavin for your patience, encouragement, and support throughout this process. 


\section{Table of Contents}

Abstract

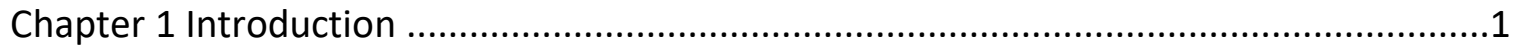

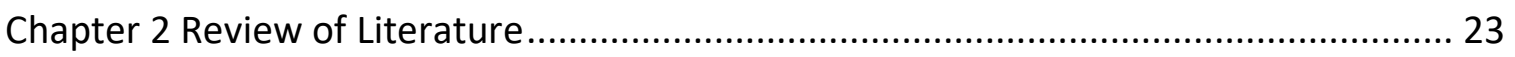

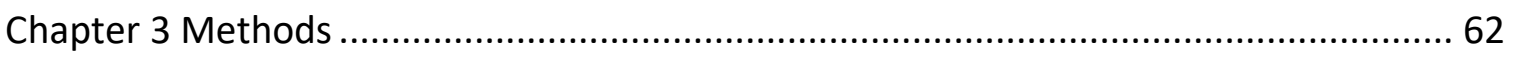

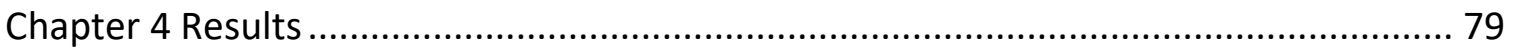

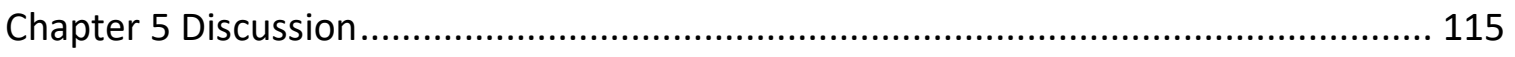

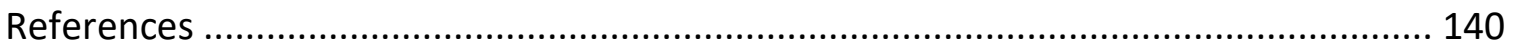

Appendix A: Invitation Letter to Superintendent ................................................. 154

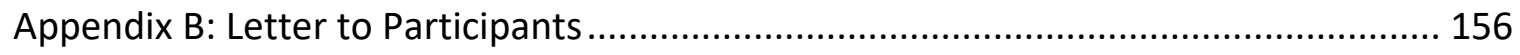

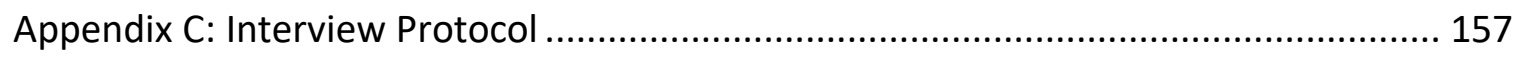

Appendix D: Informed Consent to Participate in Research ...................................... 159

Appendix E: Oregon Department of Education Survey ......................................... 162

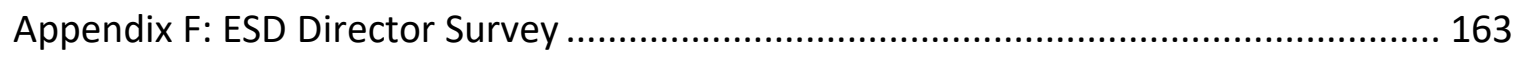




\section{List of Tables}

Table 1: Oregon School District B5 Indicator Targets for Student Placement Data (Percentage of the Day Spent in General Education Settings) for Students With

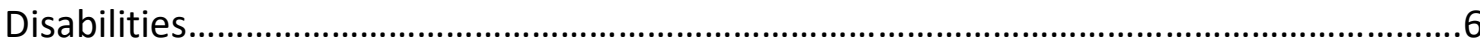

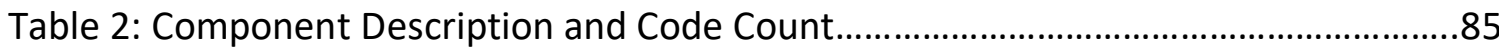




\section{List of Figures}

Figure 1: Oregon Placement Categories for Students With ID .......................................

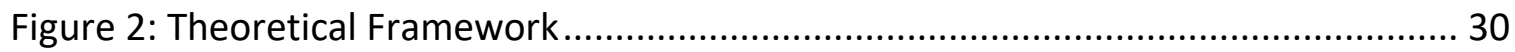

Figure 3: K-12 U.S. Placement Proportion of Students with ID, 1976 to 2014 ................. 35

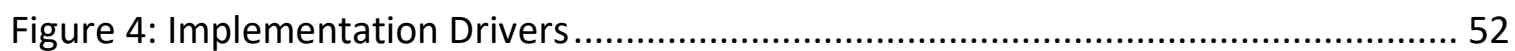

Figure 5: District 1, Federal Placement (Indicator B5) Placement for Students with ID in

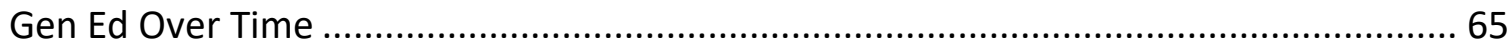

Figure 6: District 2, Federal Placement (Indicator B5) Placement for Students with ID in

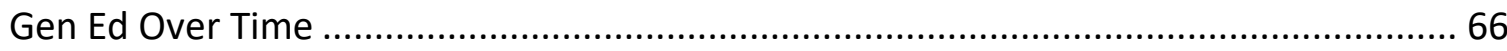

Figure 7: District 3, Federal Placement (Indicator B5) Placement for Students with ID in

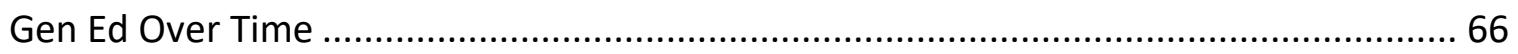

Figure 8: Total Code Co-Occurrence of Components of Loreman's (2007) Pillars ......... 100

Figure 9: Code Co-Occurrence of Components and Pillars....................................... 100 


\section{Chapter 1 Introduction}

The United States and Oregon both have a long history of segregating people with intellectual disabilities. According to the President's Committee on Mental Retardation's (1977) annual report, the identification and education of people with Intellectual Disability (ID) are documented as early as 1850. In the early 1900s, deliberate programs were put into place to "identify, segregate and sterilize every feebleminded person as a menace to social decency and racial purity: to that end that they shall not reproduce their kind" (p. 2). Segregation was supported through channeling of resources to institutions so that, by the 1970s, institutionalization was a well-established practice that was often the only available option for individuals with ID. The historical tendency has been to remove those with ID to another place. This cultural zeitgeist persists in the minds of well-meaning educators who continue to perpetuate this exclusion (Brock, 2018; Morningstar et al., 2017).

In the 1960s, the Civil Rights Movement built momentum for the rights of people whom systems had marginalized and paved the path for a new way of thinking about people with ID. The court battles in this era led to legal mandates for schools to educate all students regardless of disability, a requirement that was codified in the Education for All Handicapped Children Act of 1975. The Least Restrictive Environment (LRE) provisions have persisted in each successive reauthorization of the Individuals with Disabilities Education Act (IDEA), including the most recent authorization in 2004. Mainstreaming of students with disabilities became more popular in the 1970s and 
1980s, with special education law requiring that each student identified with ID have an individualized program to support their learning and success (Rotatori et al., 2011). Despite legal advances safeguarding the rights of students with ID and other disabilities, there remain challenges to the inclusion of students with ID in general education because of deeply held societal beliefs about people with cognitive disabilities and their ability to learn and live a full life.

The history of the Fairview Training Center, an institutionalized program for Oregonians with ID, offers a glimpse into understanding the cultural beliefs held about the capability of people with ID that, even today, results in their educational segregation. A promotional document published in 1929 describing the educational program at Fairview typifies these cultural beliefs:

Not a lot can be said for our educational department, due largely to the fact that we scarcely have adequate room or material with which to work. The feebleminded child, however, can never become independently self-supporting, and a vast sum of money and a great deal of time can be expended to no benefit in an endeavor to educate. (Ferguson et al., 2008, p. 19)

The impact of these beliefs can be traced through the history of the Fairview Training Center to the establishment of sheltered workshops as institutionalization was phased out. At the time Fairview closed in 2000, Oregon's population of people with ID had transitioned to community living-primarily in group homes-and working in sheltered workshops, performing menial labor while earning under one dollar per hour (Lane V. Brown, 2016). 
In 2012, a class-action lawsuit was brought against Oregon's Governor Kitzhaber by the plaintiff, Paula Lane, and about 7,000 Oregonians with developmental disabilities, alleging violation of the Americans with Disabilities Act (ADA) and the decision in Olmstead v. L.C., 527 U.S. 581 (1999), by segregating people with ID in settings with limited interaction with their neurotypical peers. In 2015, a settlement agreement approved by the Oregon U.S. District Court, Lane v. Brown, 166 F. Supp. 3d 1180, stipulates that Oregon will reduce the number of sheltered workshop placements incrementally over time, ensure that working-age individuals in sheltered workshops will obtain competitive integrated employment by June 2022. Further, Oregon will support employment services and training, abolish mock sheltered workshops in schools, and build capacity to train individuals with ID with the goal of competitive, integrated employment. This settlement agreement is a significant step forward towards addressing historical inequities that have plagued the state for years.

The impact of Lane v. Brown rippled through systems and agencies that serve people with ID, compelling them to shift their thinking and practice away from a deficit ideology and toward a broader view of human existence. Lane v. Brown pushed the state- toward the possibility of the dignity of risk (Schloss et al., 1993), presumed competence, and independent, dignified living. These shifted paradigms evolved conceptualization about employment possibilities for individuals with disabilities, notably ID, and support a societal view that includes a wider range of human experiences as valid and worthy. The settlement agreement specifically called out 
Oregon schools because schools were preparing most students with ID for work in sheltered workshops, not for competitive employment and independent living. From 1975 through the executed settlement agreement for Lane v. Brown, Oregon's school system sought to prepare students with ID to work in sheltered workshops. Through governors' orders and policy changes required by the settlement agreement, schools were supported to improve transition services and train for independence, integration, and opportunities for competitive employment (Lane v. Brown, 2016).

Problem Statement: The American education system systematically excludes students based on (dis)ability through its policies and ableist attitudes. Disproportionate segregation of students with ID is a form of prejudice that is acceptable today and allowable by our current laws. Fully segregated education settings for students with ID are harmful to many students with (dis)abilities, to their neurotypical peers, and to civilized society as a whole. Segregated settings for students with ID are persistent and the solution, improving inclusive educational practices, involves complex and deep systemic reform.

\section{Background of the Problem}

Under the implementing regulations for the IDEA Part B (34 CFR §300.602), each state must submit an annual report about the state's special education performance to the Secretary of Education. This report considers the state's performance relative to prescribed quantifiable and qualitative indicators in the following established priority areas: 
(1) provision of FAPE in the least restrictive environment; (2) state exercise of general supervision, including child find, effective monitoring, the use of resolution meetings, mediation, and a system of transition services . . . ; and, (3) disproportionate representation of racial and ethnic groups in special education and related services, to the extent the representation is the result of inappropriate identification. (34 CFR §300.600(d))

In addition to reporting on the state's performance relative to these indicators, each state must also analyze school district performance on each indicator. The Federal Placement Indicator (B5) is one of the indicators measuring the provision of FAPE in the LRE. This indicator tracks the percent of children with IEPs aged 6 through 21 served in different educational placements, indicative of the amount of time the student is learning in the general education environment. The first category indicates the student is learning in the general education setting for $80 \%$ or more of the day, a setting that is significantly inclusive. The next category indicates the student is learning in the general education environment less than $40 \%$ of the day, a setting that is significantly, or almost exclusively, segregated. The last category indicates the student's educational experience is entirely segregated; in other words, the student has been placed in a separate school environment, residential facility, or homebound/hospital placement without access to peers without disabilities. Each state sets an annual expected target for each performance indicator, including B5, that represents the minimum expected performance for school districts. Table 1 presents the expected targets set by the state of Oregon for the Federal Placement (B5) Indicator. 
Table 1

Oregon School District B5 Indicator Targets for Student Placement Data (Percentage of the Day Spent in General Education Settings) for Students With Disabilities

\begin{tabular}{lll}
\hline Placement & Definition & Oregon B5 Target \\
\hline Significantly Inclusive & $>80 \%$ gen ed & $73 \%$ (or more) \\
Significantly Segregated & $40-79 \%$ gen ed & \\
Almost Exclusively Segregated & $<40 \%$ gen ed & $10.6 \%$ (or less) \\
Entirely Segregated & Separate School & $1.8 \%$ (or less) \\
& & \\
\hline
\end{tabular}

Despite the Lane v. Brown settlement agreement and the directives for schools to work toward improved outcomes (moving away from training for sheltered workshops and toward more rigorous educational opportunities such as improved access to the general curriculum in the general education setting), placements for students with ID in Oregon schools remain steadfastly segregated (see Figure 1). Of the 197 school districts in Oregon, according to 2018-2019 school year data, 54 districts (27\%) did not meet the established state target for the Federal Placement (B5) indicator (Oregon Department of Education, Personal Communication, 2020) in all disability categories due to serving too many students in special education as opposed to the general education environment. In this analysis, there is one category of primary disability that illustrates an even more concerning trend. Students with ID are persistently placed in specialized programs for much of the school day. The data in Figure 1 demonstrate the significant gap between the current placement of students 
with ID compared to Oregon's Federal Placement (B5) targets. For example, in the 20192020 school year only $18.9 \%$ of students with ID were placed in Significantly Inclusive placements ( $>80 \%$ of the time in Gen Ed), well below the Oregon B5 target of $73 \%$ or more of students with disabilities. It also shows that since the Lane v. Brown settlement agreement in 2015 , the placement of students with ID has remained stagnant.

\section{Figure 1}

\section{Oregon Placements for Students With ID}

\section{Oregon Students Experiencing Intellectual Disabilities, by Federal Placement Code}

Source: https://www.oregon.gov/ode/reports-and-data/SpEdReports/Pages/default.aspx

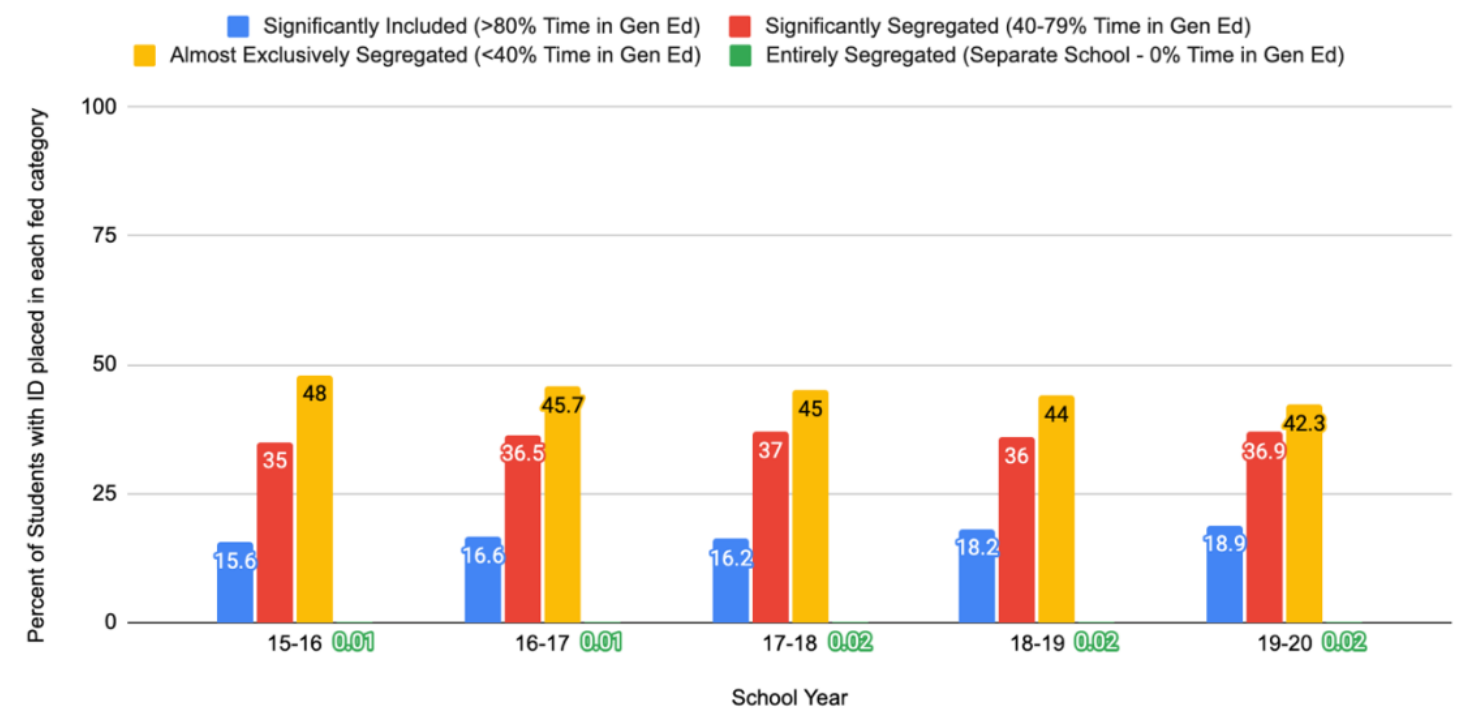

While inclusive education for students with disabilities in the United States has improved since 1975, students with ID are regularly disproportionately segregated. K-12 educational system placement practices in the United States continue to result in students with ID being educated in segregated settings (Brock, 2018; Morningstar et al., 2017). According to the U.S. National Council on Disabilities (2018), "in the 2015-2016 
school year, students with ID and multiple disabilities participated in general education classes with peers without disabilities at rates far lower than any other population ..." (p. 24). Students with ID being educated in segregated settings represents a civil rights concern because of this disparity of exclusion compared to other disability categories.

Disparities for students with ID aside, segregated placements are themselves problematic. Students with disabilities consistently experience lower achievement levels when compared to non-disabled students (Wagner et al., 2006). According to Wagner et al. (2005), students with ID are the least likely to graduate with a regular diploma and their participation rate in school, work, or training for work is the lowest of all disability categories. Further, "students with intellectual disability often experience lower rates of employment, receive lower wages, are less likely to live independently, and are less likely to attend post-secondary education than students with other types of disability" (Bouck \& Joshi, 2016, p. 154). A common assumption in special education is that segregated settings allow for a more functional curriculum (i.e., functional life skills) to be taught, which justifies placement in a separate program. However, there is not a difference for students with ID in post-school outcomes (e.g., independent living and employment for example) for students with ID when educators focused on using a functional curriculum in a separate setting versus when students with ID are educated with their non-disabled peers (Bouck, 2012). In other words, the data do not support the idea that functional skills can better be taught in a segregated setting. 
A segregated educational setting has not shown to be effective in preparing students with ID for life after high school and leads to poor outcomes for these students when they become adults (U.S. National Council on Disabilities, 2018). The President's Commission on Excellence in Special Education (2002), pointed out:

The Commission finds students with disabilities are significantly unemployed and underemployed upon leaving school compared to their peers who do not have disabilities. Too many students with disabilities leave school without successfully earning any type of diploma, and they attend post-secondary programs at rates lower than their nondisabled peers. Adults with disabilities are much less likely to be employed than adults without disabilities. Unemployment rates for working-age adults with disabilities have hovered at the 70 percent level for at least the past 12 years, which the Commission finds to be wholly unacceptable. Even when employed, too many adults with disabilities who are employed earn markedly less income than their nondisabled peers. These statistics reflect failures in the present systems' structures. We find that the overriding barrier preventing a smooth transition from high school to adult living is the fundamental failure of federal policies and programs to facilitate smooth movement for students from secondary school to competitive employment and higher education. (p. 43)

How the education system educates students with ID matters because schools are compelled to prepare students with ID for the world after graduation (Lane v. Brown, 2016). As reported by Banks and Polack (2014), "at a societal level, exclusion from education helps propagate discriminatory attitudes, creating further barriers to participation in other domains" (p. 34). As pointed out in Lane v. Brown (2016), over 7,000 Oregonians who have been, or are at risk of being, segregated in sheltered workshops have been affected by the presumption of the educational system and adult support system that segregated sheltered workshops are the destiny for people with significant cognitive disabilities. In testimony to the President's Commission on 
Excellence in Education, Wehman (2002) noted, "competitive employment history is one of the most powerful contra-indicators for youth ultimately depending on social security long-term benefits. Therefore, students need to attain competitive employment before leaving school" (p. 194). Solving this issue will require linking teaching practices, curriculum, and placement to achieve better outcomes for students with disabilities (Fisher \& Meyer, 2002; Jackson et al., 2008).

While it is a common argument that a specialized environment provides the opportunity for students with ID to receive instruction at their level, research does not bear that out. According to Kurth et al. (2016), self-contained classrooms had far less instruction and the instruction that was happening was done by teacher assistants or paraprofessionals; also, there was a high degree of distractions and fewer chances for students to respond to cues. Mcdonnell et al. (2000) found that the general education setting provided more focused instruction directed toward students with disabilities than in a segregated setting. Fisher and Meyer (2002) pointed out significant differences in measures of development and social competence given an inclusive setting. Their research demonstrates that students with severe disabilities make meaningful progress toward independence skills and social competence, key goal areas to consider for building life skills that will allow students to successfully navigate the world after their educational experience. Since Education for All Handicapped Children Act of 1975 (PL 94-142), the policy governing special education has compelled a focus on services 
and access. Only recently has the focus begun to shift toward student outcomes as the critical indicator rather than process, placement, or service delivery.

When students with ID are meaningfully included in general education classrooms and environments (conferring educational benefit and making progress towards their goals), their academic improvements are significantly higher than when educated in self-contained settings (Andresen \& Nord, 2020; Ballard \& Dymond, 2018; C. Cole et al., 2004; S. M. Cole et al., 2020; Cosier et al., 2013; Dessemontet et al., 2012; Ryndak, Alper, Ward, Storch \& Montgomery, 2010; Ryndak, Morrison \& Sommerstein, 1999; Vinodrao, 2016). For students with ID, and their general education peers, inclusive education yields improved communication, social skills, and relational benefits (Carter \& Hughes, 2005; Dessemontet \& Bless, 2013; Fisher \& Meyer, 2002; Soto, 2001 Muller et al., 2001). Perhaps most importantly, students with ID who are included in general education settings have improved outcomes in adulthood after school, including greater independence, post-school training, and competitive employment (Ryndak et al., 2012; White \& Weiner, 2004; Test et al., 2009).

How we define disability is an important nuance, yet infrequently examined. The construct of disability is rooted in a medical model that sees disability as a deficit and is inextricably linked to socio-political factors (Wehmeyer, 2019). How we define disability, and how educators think about the purpose or goal of educational services, drives how and where services are delivered. In other words, the existence of disability in a medical model presumes that there is a problem within the individual that must, therefore, be 
addressed by an expert (Symeonidou \& Phtiaka, 2009). When special education laws compelled public schools to educate students with disabilities, the goal was to move toward educational equality. This foundational idea of disability, that it is a human deficit, set the stage for how we think about special education services today; IDEA conceptualizes disability based on a medical model. Ableist educational systems are the natural result of the deficit mindset inherent in a medical model of disability being used to frame educational policy.

While traditional views of disability in the 1970s helped further the agenda for educational equality, this viewpoint has since stymied efforts to bring about acceptance of people with cognitive disabilities as equal and valued members of society (Agmon et al., 2016). The field of special education has wrestled with how disability is conceptualized, touting two different viewpoints made up of incrementalists and reconceptualists (Baglieri, Valle et al., 2011). Incrementalists work out of the medical model of disability and purport the purpose of special education is to fix the problem within the person. Reconceptionalists, on the other hand, view disability as a social construction and address disability by reshaping the context (Andrews et al., 2000; Baglieri, Bejoian et al., 2011). There is value in both perspectives: we must actively teach skills in school to students experiencing disability not to fix them, but to support their access to the general curriculum and to prepare them for life after school, and we must push towards improving ableist aspects of education that continue to be discriminatory in practice, such as the disproportionate segregation of students with ID. 


\section{Statement of the Research Problem}

IDEA considers improving outcomes for students with disabilities an "essential element of [the United States] national policy" (20 U.S.C. § 1400). The preponderance of the available research over the past 30 years reveals that inclusive practices support improved outcomes (Sailor, 2016; SWIFT, 2017). Yet, despite increased inclusion being the lever that can enable improved outcomes, historical, contextual, and attitudinal issues continue to perpetuate the exclusion of students with ID. The U.S. education system, through its policies, ableist attitudes, and practices, by the systematic exclusion of students with ID maintains a system of segregation and limits opportunity for access to more rigorous content. Disproportionate segregation of students with ID is a form of prejudice that is acceptable today and allowable under federal, state, and local policies. Segregated placements for students with ID are harmful to students with disabilities and to their neurotypical peers.

The disproportionate placement of students with ID in segregated spaces is ableist. While the practice of inclusive placement in the general education setting is encouraged by federal and state law and administrative rule, there appear to be assumptions about student ability, an example of ableism, that often lead to decisions to place students with ID in segregated placements (Agran et al., 2020). In other words, well-meaning educators often presume that a student is a candidate for a specialized program because of a disability label, or a score on a standardized test. Deficit thinking can often limit or eliminate discourse and wondering about how a more rigorous 
educational program in the general education setting could benefit a student with ID (Sauer \& Jorgenson, 2016). The purpose of this study is to better understand how leadership practices in schools have led to improvement in inclusive practices which leads to more rigorous instruction in general education settings and improved outcomes.

\section{Significance of the Research Problem}

Students with disabilities are entitled to an appropriately ambitious educational program that takes into account their potential for growth and affords them the chance to meet challenging objectives (Agran et al., 2020; Wehmeyer, 2019). The general education classroom, with appropriate support, has been shown to confer more benefit for students with ID because it increases access to the general education curriculum (Jackson et al., 2008; Soukup et al., 2007), content expertise (Kleinert et al., 2015), and peer supports (Carter \& Hughes, 2006; Helmstetter et al., 199; Hornby, 20148). Despite this evolution of research-based practice, the segregation of students with ID persists. In other words, though both litigation and scholarship have compelled a higher standard of practice, inclusive settings that can provide that higher standard of practice are too often denied to students with ID. The IDEA compels districts to offer a free appropriate public education (FAPE) and to provide special education services in the LRE. Yet, students with ID are disproportionately segregated in schools, presenting a significant problem of practice in a field that has widely recognized the value of inclusion. 
Educational equity reform in a democratic society is the primary work of its educational institutions. If the goal of public education broadly is to ensure each student, regardless of ability or background, can benefit from and prepare for full participation in civic life, schools must strive to identify students who are not successful in achieving their educational goals and reform policies and practices to enable all students to benefit from a high-quality education. The Oregon Department of Education outlines placement targets for students with disabilities (see Table 1), and many districts fall short. Segregated classrooms, while allowable under the law, are harmful to students with and without disabilities. By investigating how leaders in districts where improvement has been demonstrated are able to move from segregated placements to inclusive practices for students with ID, this study hopes to provide insight for other leaders seeking to address this problem.

This study investigates connections between the problem of disproportionate segregation of students with ID to shifts in thinking, attitudes, structures (systems), and practices by leaders that led to more inclusive placements. IEP placement decisions are made by teams of educators and parents within a context of historically ableist policies and practices. Bias and perceptions of disability are significant determinants in the placement of students with ID in segregated settings (Agran et al., 2020). Educators' objections to inclusive placement for students with ID form around the ableist presumption that "all who portray these disability characteristics should be educated in the same manner and in accordance with the foregoing stereotypes of their 
inadequacies" (Agran et al., 2020, p. 6). This presumption, common in education, leads to a de facto policy of placement decisions made by categorization rather than individual student needs (Brock, 2018; Morningstar et al., 2017).

In order to increase inclusive teaching and learning for students with ID in our K12 systems, school district leaders must not only consider how to address ableist beliefs, and behaviors toward the child/student but, as Avramidis and Norwich (2002) suggested, they must address the system as a whole. In other words, to change educator attitudes, system structures must be addressed comprehensively. Loreman (2007) posited a framework of seven pillars for implementing inclusion in schools. These pillars are: (a) Positive Attitudes, (b) Policy and Leadership, (c) School and Classroom Processes, (d) Curriculum and Pedagogy, (e) The Community, (f) Meaningful Reflection, and (g) Training and Resources. Loreman's first pillar suggests that developing positive attitudes is "central to the accomplishment of inclusive education" (p. 24). Each of Loreman's seven pillars of inclusion can be viewed in relation to the core tenet of educator attitudes spanning child, teacher, and environmental support variables. Addressing educator attitudes comprehensively within the context of implementing inclusion, then, requires schools to respond to child-related, teacher-related, and environmental support-related factors (Avramidis \& Norwich, 2002). Loreman's framework will be used as an organizational guide for understanding how school district leaders describe efforts to support increased inclusion of students with ID in general education classrooms. 


\section{Methods and Research Question(s)}

The purpose of this study is to understand how district and school leaders (in thought, planning, and action) move away from segregated settings and improve inclusive education for students with ID, thereby increasing the inclusive placements of students with ID, leading to meaningful participation in the general education setting, access to more rigorous instruction and improved outcomes. This study selected three Oregon school districts that are improving inclusive education for students with ID and examined how leaders improved the metric of placement. The phenomena to be studied are leadership thinking, planning, and actions within a bounded system of a school district. The methodology for this study is qualitative in nature because the research questions posed in this study are process questions (i.e., how do they improve?). An explanatory multiple-case study design was selected because the unit of measure (school districts) is a bounded system and the questions seek to understand how and why. Districts were selected as the primary identified unit of measure in order to: (a) compare various priorities for implementing inclusive practices between bounded systems; and (b) consider the connection between local community values and district policies, practices, and procedures impacting inclusion as a factor leading to improved rates of inclusion for students with ID. As district size and location present unique challenges to inclusion for students with ID, the districts selected represent a range in size and geography to increase validity of the findings. 


\section{Data Collection and Analysis}

Data collection consisted of interviews with leaders in selected districts and schools, and gathering of artifacts that guide policy and practice relevant to placement for students with ID. Interviews were transcribed, coded, and analyzed to answer the research questions, guided by the theoretical frameworks (i.e., Avramadis \& Norwich, 2002; Loreman, 2007). The data analysis strategy utilized Pattern-Matching (Yin, 2018) and Pattern Coding (Saldaña, 2021) within and between the cases (districts) to answer the research questions.

\section{Research Questions}

The primary research question addressed in this study is: What do leaders in school districts successfully increasing inclusion of students with ID attribute to supporting increased inclusion of students with ID? In order to deepen understanding related to the primary research question, the following questions informed the study:

1. What are the commonalities and differences in Components (systems, processes, and practices) that administrators identify to promote the inclusion of students with ID in the general education classroom?

2. What is the relationship between Loreman's (2007) Pillars and Components (systems, processes, and practices) that leaders describe contribute to students with ID being educated in the general education classroom?

3. How do leaders across different district contexts describe (1) their own attitudes and (2) educator attitudes about the inclusion of students with ID and the district's efforts to address those attitudes? 


\section{Key Concepts}

Ableism emphasizes discrimination in favor of non-disabled people. It is a form of discrimination or prejudice against individuals with physical, mental, or developmental disabilities. The concept promotes certain abilities and characteristics over others, like productivity, competitiveness over others such as empathy, compassion, and kindness (Wolbring, 2008). Another aspect is preferential hegemonies driven by the belief that individuals with disabilities need to be fixed or cannot function as full members of society (Castaneda \& Peters, 2000).

Child-Related Variables: Teacher perception of the child and their disabling condition is a factor influencing teacher attitudes toward inclusive education for students with disabilities (Avramadis \& Norwich, 2002).

Deficit Ideology: The belief that inequalities result from presumed intellectual, moral, cultural, and behavioral deficiencies, rather than from unjust and oppressive social conditions, such as systemic racism, ableism, sexism, or economic injustice (Gorski, 2011).

Disablism emphasizes discrimination against disabled people. Under disablism, discriminatory, oppressive, and abusive behavior arises from the belief that people experiencing disability are inferior to others. Disablism refers to institutional discrimination of people because of actual or perceived disabilities (F. A. Campbell \& Kumari, 2008). 
Differentiated Instruction, or Differentiation, is an approach whereby teachers adjust their curriculum and instruction to maximize the learning of all students: "average" learners, emergent bilinguals, struggling students, students with learning disabilities, and gifted and talented students. Differentiated instruction is not a single strategy but, rather, a framework that teachers can use to implement a variety of strategies, many of which are evidence-based. Frequent evidence-based strategies used for differentiation include:

- Employing effective classroom management procedures;

- Grouping students for instruction (especially students with significant learning problems);

- Assessing readiness; and

- Teaching to the student's zone of proximal development.

Environment-Related Variables in the school setting, such as administrative support and school climate, influence teacher attitudes towards inclusion of students with disabilities.

Inclusion occurs when a student with a disability is receiving their education in a general education regular class setting, reflecting natural proportions and ageappropriate heterogeneous groups in core academic and elective or special areas within the school community. In inclusive systems, a student with a disability is a valued member of the classroom and school community; the teachers and administrators support universal education and have knowledge and support available to enable them to effectively teach all children; and access is provided to technical assistance in best 
practices, instructional methods, and supports tailored to the student's needs based on current research (Florida Statute Section 1003.57(1)(a)(2)).

Inclusive Classroom: An inclusive classroom is an educational setting for students with and without disabilities, where all of the supports and services needed for all of the students to succeed are provided (Boston-Kemple, 2012, p. 110).

Inclusive Practices: "an ongoing struggle toward (a) the redistribution of access to and participation in quality opportunities to learn, (b) the recognition and valuing of all student differences as reflected in content, pedagogy and assessment tools, and (c) the creation of more opportunities for non-dominant groups to advance claims of educational exclusion and their respective solutions" (Waitoller \& Artiles, 2013, p. 8).

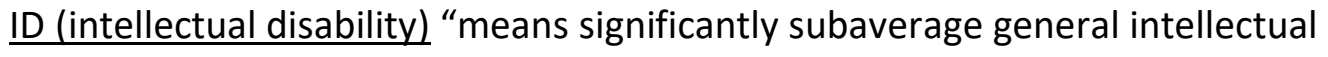
functioning, existing concurrently with deficits in adaptive behavior and manifested during the developmental period, that adversely affects a child's educational performance" (34 CFR § 300.8(c)(6)).

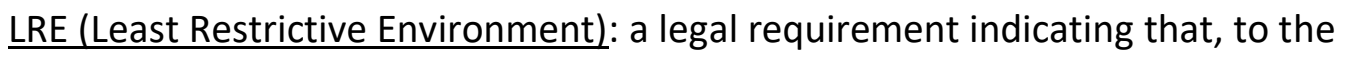
maximum extent appropriate, children with disabilities, including children in public or private institutions or other care facilities, are educated with children who are nondisabled; and special classes, separate schooling, or other removal of children with disabilities from the regular educational environment occurs only if the nature or severity of the disability is such that education in regular classes with the use of 
supplementary aids and services cannot be achieved satisfactorily (IDEA, 34 CFR 300.114).

Primary Driver: A hypothesized lever in organizational change models in the context of a driver diagram in improvement science (Bryk et al., 2017). Primary drivers are key influencing factors that influence change in a significant way.

Secondary Driver: A set of factors that influence the primary driver in the improvement science literature (Bryk et al., 2017). Change efforts often begin with different inputs to secondary drivers that then influence the primary driver that impacts the larger goal or aim.

Sheltered Workshop: refers to an organization or environment that employs people with disabilities separately from others. The origin is from the outgrowth of institutions for people with disabilities (Disabled World, 2019).

Teacher-Related Variables: such as gender, time in service, grade level taught, training, experience teaching students with disabilities, and beliefs influence teacher attitude toward inclusion of students with disabilities in the general education setting (Avramadis \& Norwich, 2002). 


\section{Chapter 2 Review of Literature}

The problem under review in this study is that students with ID are segregated from their neurotypical peers to receive their education. The IDEA compels schools and districts to offer a continuum of alternative placements (34 CFR § 300.115) when education in the general education setting, even with supports, does not confer meaningful benefit. However, the data (national, state, and local) suggest that for students with ID, the number of students in segregated settings is disproportionately higher when compared to other disability types. This problem is rooted in how disability is viewed, especially the ongoing impact of the foundational deficit-framing of the IDEA (Wehmeyer, 2019); lagging inclusive instructional practices, such as the under-adoption of frameworks like Universal Design for Learning (UDL) that can provide better support for all learners in general education settings (Fritzgerald, 2021); and unexamined ableist views that limit educators' and societal expectations for people with ID (Voulgarides, 2018). The focus of this study is to identify and examine exemplar districts in Oregon in order to understand how they have made progress in their schools toward inclusive education for students with ID.

It is important to note that, while placing a student experiencing disability in the regular education setting may improve s districts' performance relative to the Federal Placement Indicator (B5), improvement in this indicator does not necessarily mean that effective inclusive education is taking place for students with ID. Regardless, effective inclusive education is certainly not occurring in segregated spaces. Therefore, the 
Federal Placement Indicator (B5) is the most appropriate data point for entry to explore the dynamics and conditions in learning organizations that allow for the reduction of segregated education based on ability, as a prerequisite to effective inclusion.

\section{Theoretical Framework}

This study anchored the analysis of the data collected in three theoretical frameworks that together allowed deep exploration of this problem. These frameworks are: (a) Disability Studies (Baglieri, Bejoian et al., 2011; Baglieri, Valle et al., 2011; Davis, 2017; Watson et al., 2012); (b) seven pillars for inclusion (Loreman, 2007); and (c) variables on addressing attitudinal barriers (Avramidis \& Norwich, 2002). Rooting the analysis in the framework of Disability Studies connects the dynamic presence of ableism and deficit ideology which contributes to understanding attitudinal barriers to implementing inclusion. The additional frameworks deepened the analysis by examining factors that can improve inclusive educational practices and the variables involved in addressing educator attitudes. Loreman's (2007) Seven Pillars for Inclusion is a framework for improving inclusive practices. Avramidis and Norwich (2002) identified contributing attitudinal variables that helped or hindered implementation of inclusive education policies and practices. Both frameworks offered lenses through which to conduct analysis in this study in order to answer the research questions.

\section{Disability Studies, Ableism, and Deficit Ideology}

The field of Disability Studies (DS) has helped shift thinking about disability in the sociopolitical realm by introducing an alternative viewpoint to the medical or deficit 
model of disability. This shift has direct implications for how we view people experiencing disability and offers another view, a social model. The social model of disability shifts thinking away from a "problem" residing within an individual to focus on the interaction between the person and the environment in which they reside. In a social model of disability, then, disability is not a personal deficit, but the result of people with diverse needs living in an ableist world. In DS, impairment refers to the actual attributes or lack of attributes that affect a person, and that disability is a restriction caused by society and the environment when it does not accommodate the needs of individuals with impairments. DS scholarship posits that disability "should be seen as a political and socially constructed problem with a focus on the disabling barriers faced by people with an impairment" (Watson et al., 2012, p. 3). Broadly speaking, DS is construed to have three core tenets: (a) people experiencing disability are marginalized; (b) people experiencing disability constitute a minority group; and (c) disability can be reconstructed from a medical problem to a social problem. DS sees disability as a matter of social discrimination as opposed to an individual deficit (Watson et al., 2012). Disability studies as a scholarship field help reframe the presumptions and beliefs that contribute to attitudinal barriers, such as ableism.

Ableism is "a pervasive system of discrimination and exclusion that oppresses people who have mental, emotional and physical disabilities" (Rauscher \& McClintock, 1997, p. 198). Yet, ableism "is often unrecognized or overlooked in analyzing why students with disabilities have difficulties being included" (Storey, 2007, p. 56). Ableism 
may remain unrecognized because it is "so enmeshed in the fabric of our social order ... [that it] appear[s] both normal and natural to people in this culture" (Delgado \& Stefancic, 2001, as cited in Annamma et al., 2013, p. 6). Ableism rests on societal attitudes that uncritically assert that it is better for a child to walk than roll, speak than sign, read print than read Braille, spell independently than use a spell-check, and hang out with nondisabled kids as opposed to other disabled kids, etc. In short, in the eyes of many educators and society, it is preferable for disabled students to do things in the same manner as nondisabled kids. (Hehir, 2002, p. 3)

Ableism persists because of bias (both conscious and implicit) that values or favors certain characteristics or abilities over others (Wolbring, 2008). Similarly to racism, a key nuance with ableism is that power is structurally entrenched in ways that privilege and uphold ability over disability (Annamma et al., 2013). The concept of ableism "describes, and is reflected in, individuals and group perceptions of certain abilities as essential. Ableism can be treated as both a hegemony which promotes ability preference and as an analytical tool used to understand these preferences and their impact[emphasis added]" (Hutchen \& Wolbring, 2012, p. 40). In other words, applying ableism within the conceptual frame of disability studies, can increase understanding of the underlying attitudinal structures that influence policy, process, and practice that keeps the notion of segregated schooling acceptable.

In the context of this study, ableism is examined as a belief system resulting in a deficit ideology for people with ID; supporting the notion that people experiencing disability need a special, separate place \& treatment (Agran et al., 2020). Ableism is perpetuated by the continued conceptualization of disability through the deficit-driven 
medical model. It is predicated on the premise that people with ID are inherently broken, or impaired, and thus are not equal in value to other neurotypical human beings. Under that premise, it becomes essential that the impairment be "fixed," and the "fixing" needs to occur with a specialist, outside of the regular setting. We exclude and segregate people with ID because of unexamined assumptions about their capability, their potential, and their value; and special education policy is written on the premise of the medical, or deficit, model of disability. Unexamined ableism has been and is used to justify the exclusion of people with differing abilities over time and across cultures.

Ableism is closely linked to deficit ideology because beliefs influence decisions and practice. An example of this connection can be observed in placement decisions for a student with ID in the IEP process. If team members believe that the student has a neurological impairment (ID) and, because of that impairment, the student is not competent enough to join the rigor of the general education setting, ableism is functioning as the barrier to inclusion. It acts as a force that allows a deficit ideology to segregate the child. Looking at this problem through the lens of ableism helps frame the attitudinal and ideological barriers that exist when teams are making a decision about where a student with ID should receive their education. Ableism is pervasive in our social institutions and too-often persists unexamined, particularly when nondisabled people lack exposure and positive experiences with people who experience disability (Bogart \& Dunn, 2019). By understanding the dynamics of ableism as an institutional 
undercurrent, we can identify structures that perpetuate the bias and address them through systems change. It is important to understand ableism within the context of this study because it helps explain why segregated placements have remained an intractable problem in special education.

The intersectionality of disability and race also play an important role when considering bias. Whereas ableism is a form of discrimination based on disability-based bias, race-based bias also results in an overrepresentation of Black and Hispanic students with disabilities in segregated settings (Grindal et al., 2019). When they examined data from three states, Grindal et al. (2019) found that, controlled for socioeconomic variables, race played a role in which students were placed in a substantially separate setting. The authors in this study pointed out that Black and Hispanic students "may be referred for special education evaluation[s] because teachers inappropriately perceive them to have more inherent difficulty behaving or have lower academic skills" (Grindal et al., 2019, p. 526). They posit that these perceptions may be due to teachers viewing special education as a resource for supporting students and an opportunity for additional help. The role of race as a determinant of overrepresentation of students with ID in segregated classrooms is well documented (Connor \& Ferri, 2005; Donovan \& Cross, 2002; Fierros \& Conroy, 2002; National Council on Disability, 2015) and raises the critical importance of understanding the dynamic tension racial and ability bias play into decisions to place students separately from their peers. 


\section{Loreman's Seven Pillars for Inclusive Practice}

Loreman (2007) proposed his framework to shift the discussion and scholarship

away from a focus on justifying inclusive education and towards a focus on work that

will increase inclusive practices in the classroom, based on research identifying the

conditions necessary to support meaningful inclusion (see Figure 2). Loreman's pillars

are a frame to view the necessary elements in a school and district setting to support

and sustain inclusive education. The seven pillars are:

1. Developing positive attitudes. Positive attitudes about disability that can challenge deficit thinking is essential. Both educators and the larger school community must be willing to address deficit ideology and ableism when it impacts the school community. The minute-by-minute decisions made by implementing staff makes the difference in developing and sustaining meaningful inclusive education (Loreman, 2007).

2. Supportive policy and leadership. Leadership at all levels-from the school board, to district-level leadership (e.g., superintendents, directors), to school- and classroom-level leadership (e.g., principals and administrators, teachers) - must believe in the value of inclusive education and have sufficient commitment to overcome ableist thinking and other implementation challenges (Loreman, 2007).

3. School and classroom process grounded in research-based practice. The evidence-base to practice gap must be addressed as outdated methods simply do not yield the instructional practices needed to meet the learning needs of students with cognitive and other disabilities (Loreman, 2007).

4. Flexible curriculum and pedagogy. A school system must embrace a studentcentered approach that supports a wide range of student need, such as UDL (Meyer et al., 2014). Pedagogically, teachers must end the use of ability grouping and instead implement multiple means of representation, engagement, and action and expression in order to meet the needs of all learners within mixed-ability groups (Loreman, 2007).

5. Community involvement. Community engagement is a key support element in inclusive education. Specifically, bringing parents in as decision-makers in meaningful ways, recognizing parents as their children's first teacher, and seeing parents as advocates for their children are critical ways schools can involve families. This pillar also suggests other agencies are necessary as 
partners in the work (e.g., advocacy and parent education groups) (Loreman, 2007).

6. Meaningful reflection. Inclusive schools must foster a climate of reflection, whereby leadership encourages, coaches, and builds time for reflective practice. This cycle of practice, data, and reflection is needed for the adaptive growth and change that will support the ongoing reforms needed for inclusion to become successful (Loreman, 2007).

7. Training and resources. Training and resources are necessary for general education teachers to have the skills to support inclusive education effectively. Partnership with local universities or regular school-led professional development is required. Significant resources-for example, direct support in the classroom, expert consultation, time to collaborate, and assistive technology-are necessary to include all learners in the general education classroom appropriately (Loreman, 2007).

\section{Figure 2}

\section{Theoretical Framework}

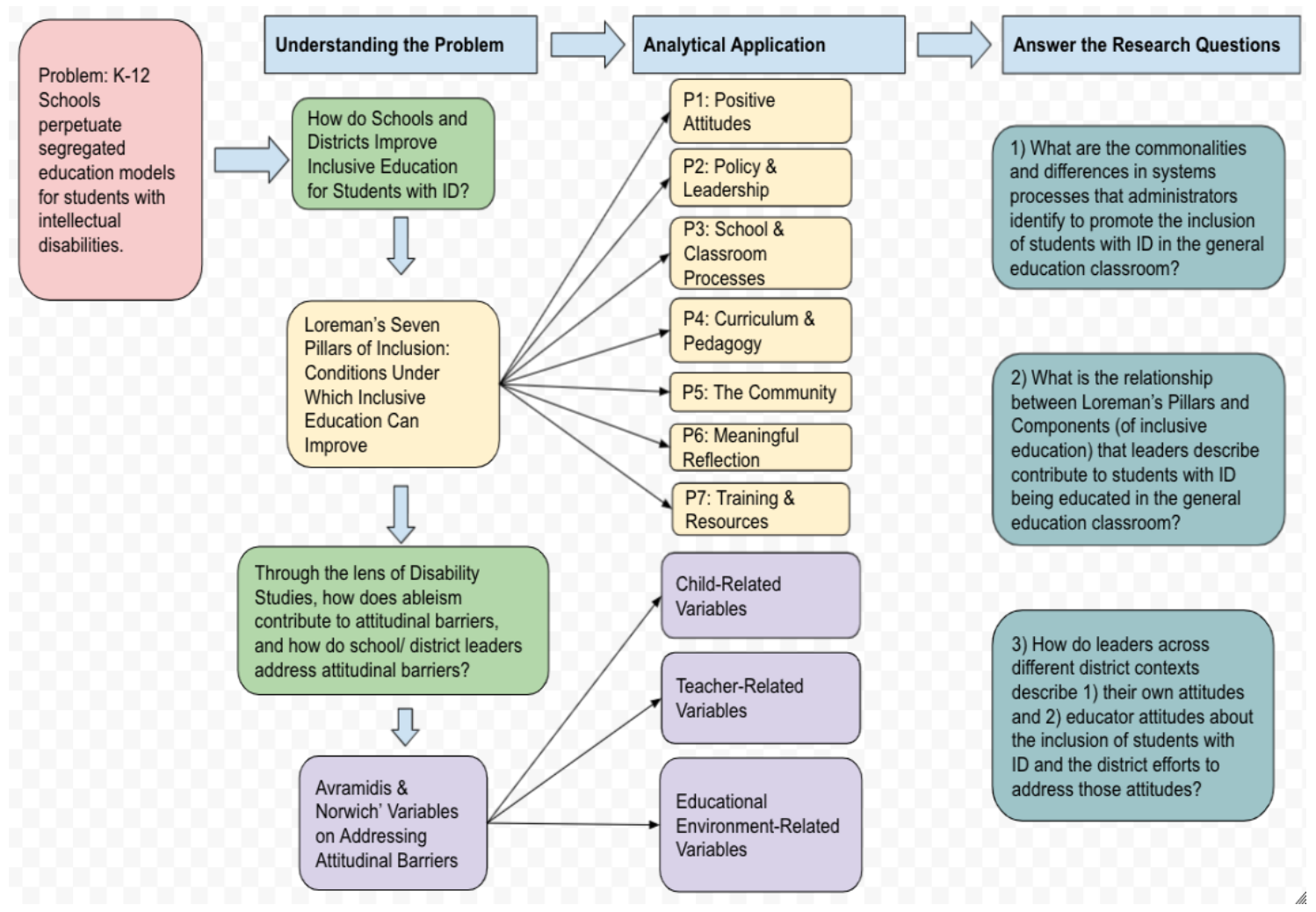


Segregated placements for students with ID persist throughout Oregon's K-12 education system. Ableism and deficit ideology likely play a role in the persistence of this problem. Adaptive problems influenced by bias (i.e., ableism and deficit ideology) can be effectively addressed through the variables that influence attitude (Avramidis \& Norwich, 2002). Viewing this problem from the perspective of the influence of disability studies, ableism, deficit ideology, and the historical context provides a better understanding of how bias acts as a contributing factor in the perpetuation of segregated spaces for students with ID. In other words, ableism and deficit ideology contribute to educators' negative perception of disability, but this can be improved by working through variables addressing attitudinal barriers. Developing positive attitudes is essential foundational work to improve inclusion. Loreman's (2007) seven pillars recognize this, and provide a framework that moves beyond attitudinal barriers to understand the systems and structures that can support the implementation of inclusion. It is this convergence of understanding the persistence of segregated placements and a framework for moving toward improvement in education that enables this study's focus on exploring settings where the problem is being intentionally addressed.

By focusing on ableism and educator attitudes as a contributor to persistent segregated placements for students with ID, this study may miss other significant contributing factors. Segregated placements for students with ID is a significant, complex problem that likely has multiple, perhaps interrelated, causes. Lives are 
impacted by persistent, segregated schooling for students with ID. Given that, there is risk in viewing ableism and negative attitudes as the primary factors contributing to segregated placements. Problem solving models such as Improvement Science (Bryk et al., 2017) inform how to plan interventions based on the underlying barrier which means that, if segregated settings for students with ID are being inappropriately attributed to ableism, the wrong solutions will be developed.

Given this risk, significant effort was put into considering the potential contributing factors to segregated placements for students with ID and determining the appropriate theoretical frameworks to underpin the study. In early iterations of this study design, multiple theoretical and conceptual frameworks were investigated, including critical theories that examined power structures and the intersectionality of race and disability. For instance, DisCrit is a recent theory that outlines how Critical Race Theory and Disability Studies (Annamma et al., 2013) can help frame the dynamics involved. In designing this study, I worked to select a conceptual framework that could support understanding of the dynamic tension and psychological factors that allow the practice of segregation to be a prevalent problem.

Though many frameworks could be used to explore this problem, in this case, ableism through the lens of disability studies as a conceptual framework allowed this tension to surface enough for analysis and understanding of the primary root cause of the problem of segregation based on presumptions of ability. Given the likelihood of other dynamics being involved in this problem's persistence, selection of disability 
studies as a conceptual framework presented a risk that analysis of one or more other contributing factors would not be fully examined (e.g., the intersectionality of oppression because of disability and race is important, given the deep history of segregation based on race, and would be thoroughly considered through a DisCrit lens). Further, by looking to disability studies and ableism as a contributing factor to the stated problem, this study may miss other factors such as ineffective teaching practices connected to the persistent failure for students with ID that may lead to the perpetuation of ableism in regards to students with ID.

I attempted to mediate this risk through selection of Loreman's (2007) Seven Pillars and Avramidis and Norwich's (2002) Variables on Addressing Attitudinal Barriers as complementary theoretical frameworks. Loreman's Seven Pillars offers a framework for necessary organizational components needed to create the conditions for improvement of the problem of segregated placements for students with ID. Avramidis and Norwich offered a framework in which to view addressing educator attitude. However, these theoretical frames assume a level of comprehensiveness that could miss important considerations in improving inclusive practices in schools. If faulty, these presumptions would hinder the work. The risk of selecting these theoretical frameworks to examine how leaders describe efforts to improve inclusion is that important considerations may be missed.

There are other frameworks, such as the SWIFT Schools Framework supported by the SWIFT Education Center at the University of Kansas (SWIFT, 2020), and the 
Inclusive Education Framework (2020) out of the NCSE in Ireland, that could have been selected to frame the analysis in this study. Careful consideration of available options resulted in selection of Loreman's (2007) and Avramidis and Norwich's (2002) frameworks. This study will highlight other themes that emerge from the data collection, even where they do not align with the selected frameworks, to help control for this possibility.

\section{Review of the Literature}

Segregated school placements for students with ID are a persistent and substantial problem in Oregon and throughout the United States (Kleinert et al., 2015). While the IDEA compels districts to offer a continuum of placement options for students with disabilities, research indicates (Agran et al.,2020; Brock, 2018; Morningstar et al., 2017) that students with ID are disproportionately placed in segregated settings.

Despite advances in evidence-based frameworks and teaching practices like UDL (Meyer et al., 2014; Rose, 2013) to create educational access for a wider range of student learning needs, the problem persists (see Figure 2 ) at both the local and the national levels (Brock, 2018). 


\section{Figure 3}

K-12 U.S. Placement Proportion of Students With ID, 1976 to 2014

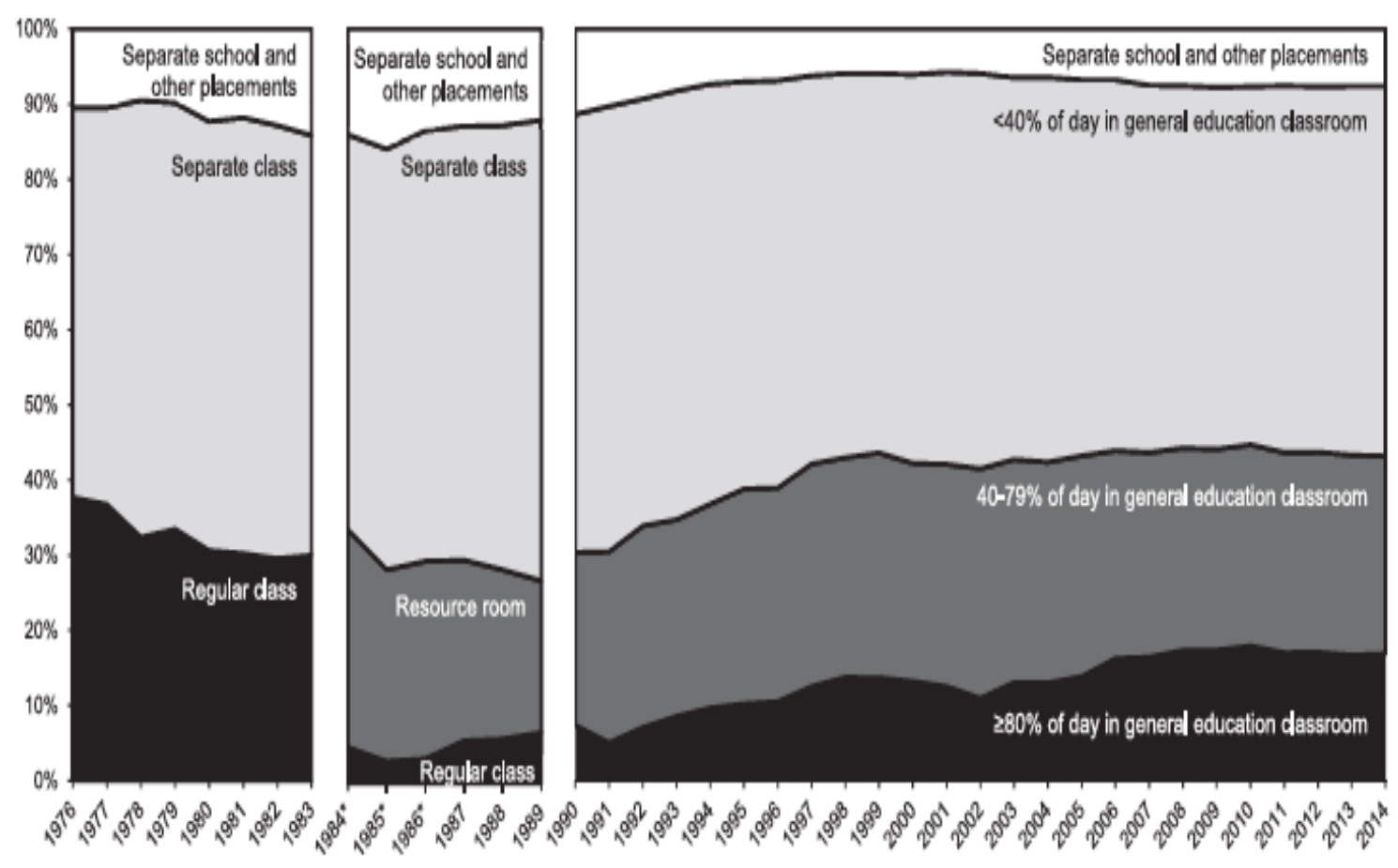

Source: Brock (2018).

Policy decisions in our educational system are often based on the assumption that there is statistical normalcy inherent in nature (Baglieri, Bejoian et al., 2011). School systems (buildings, curriculum, instructional strategies, and placement options) are designed to fit the myth of the average learner (Rose, 2016). The concept of normal, or average, is a construct first emerging in the scientific literature in the early 17th century by a French statistician, Quetelet, who argued that applying laws of errors from astronomy, and by averaging variables of height and weight, we could formulate the average human (Davis, 2017). The effort to define the average human significantly 
impacted the structures of public education, as "the concept of a norm, unlike that of an ideal, implies that the majority of the population must or should somehow be part of the norm" (Davis, 2017, p. 26). Thus, psychometrics were born; consequently, all facets of modern life, including the public education system, have been designed for or around normative statistics. Applying psychometrics to the concept of human variation is the basis for constructing and defining disability: those who do not meet or exceed the norm, or average, must be "less than." They must be disabled. There is significant unexamined ableism that allows this deficit paradigm to persist.

Unexamined ableism can lead to discrimination and social prejudice against those with disabilities. It results from an unexamined schema of the world predicated on the myth of average by holding a bias against those who do not fit the norm. The hegemony of ableism is a hidden and often subconscious power structure in place that protects the needs of the dominant majority (Kress-White, 2009). Deficit thinking or ideology is the manifestation of ableism when applied to the consideration of access to school for children with disabilities. As Valencia (1997) points out, deficit thinking leads to locating, "the blame for the problem . . . by the more powerful party-in the individual person, the victim - rather than [within] the structural problems of the unit" (p. x). Ableism and deficit thinking (ideology) is a pervasive problem that leads to higher rates of exclusion of students with ID. As the field of Disability Studies points out, like racism, ableism upholds the notion of defining normal (Davis, 2017). Annamma et al. (2013) proposed DisCrit partially in recognition of the idea "that normative cultural 
standards such as whiteness and ability lead to viewing differences among certain individuals as deficits" (p. 12).

There is a distinct connection between ableism and attitudes educators may hold toward students of difference, outside of what is believed to be normal. For example, Gilmore et al. (2010) reported that their study respondents (231 teacher trainees) had reservations about having a student with Down Syndrome in their class, displaying a low level of understanding about the syndrome and its impact on developmental milestones and lifespan of impacted children. Gilmore et al. (2010) purported that

studies of teacher attitudes towards inclusive education have generally found that, despite overall support for the concept of inclusion, the majority of teachers feel that the regular classroom is not the best option for children with disabilities and their views on inclusion become less positive with increasing years of classroom experience. (p. 66)

Attitudes are the first place where ableism and deficit ideology show up, which is a determinant in the educational decision-making and placement process (Agran et al., 2020) and is an indicator of the adaptive shifts that need to be made to address the problem of segregation based on ability. Deficit ideology is an unchecked presumption, or attribution error, that mistakes difference from ourselves for deficit (Gorski, 2011). As it relates to the problem in this study, if educators and those in the educational community continue to conceive of difference as deficit, a movement toward inclusive education will be difficult. Movement towards inclusion is stymied without addressing attitudes (Loreman, 2007). As Artiles et al. (2010) suggested, "there is evidence that placement patterns can be moderated by structural factors and large-scale reforms, as 
well as by the culture of schools and the values, attitudes, and capacities of administrators and school staff" (p. 281). Addressing educator attitudes is central and core to sustainable improvement in including students with ID in the general education setting. It is important to better understand how attitudinal shifts are understood and addressed by districts successfully including students with ID in the general education classroom. School leaders must not only hold but model a positive attitude toward inclusion, for it "is the principal who sets the tone for the entire school community" (Horrocks et al., 2008, p. 1472). Therefore, the attitude of school leadership is a key for inclusive practice. As Bennett and Gallagher (2013) pointed out, principals lead and model "beliefs that all students can learn, and ownership of student learning by the teacher" (p. 99).

\section{Developing Positive Attitudes}

Educator attitudes are an essential component to address when considering the larger goal of improving inclusive practices at school (Monsen \& Frederickson, 2004). Loreman (2007) identified developing positive attitudes of the educators and community as essential in order to increase the inclusion of students with disabilities and address the underlying deficit ideology and ableism that perpetuates segregated settings. Given that "attitudes reflect an individual's global positive or negative beliefs about a particular behaviour [sic], issue or policy" (Sosu \& Rydzewska, 2017, p. 3), an individual's core beliefs have a clear connection to their attitude towards inclusion. Silverman (2007) examined the relationship between a person's epistemological beliefs 
and their attitudes toward inclusion. Perhaps unsurprisingly, effective teachers of students with disabilities in general education classrooms "believe that learning ability is improvable and accept the slow and effortful nature of learning for some students" (Silverman, 2007, p. 43). Attitude is an aggregation of beliefs, which makes beliefs critical to inclusive practice. M. R. Olson et al. (1997) reported that teachers who were successful in teaching students with disabilities in the general education classroom express a sense of responsibility for all students and self-describe as tolerant, reflective, warm, and responsible.

When considering how to support inclusion, Avramidis and Norwich (2002) referred to ableism as an attitude impacting child-related variables (i.e., teacher perception of the child), such as disability labels. This impact of ableism also informs how the teacher understands the child's disability. Avramidis and Norwich recognized the critical need to address individuals' attitudes about child-related variables, but they also identify two additional sets of variables that need to be addressed to support and sustain successful inclusion: teacher-related variables (i.e., preparation of the teacher) and educational environment-related variables (i.e., supports to the teacher). They suggest that, if educators only address attitudes concerning child-related variables, the attitudinal changes and inclusion of the student are unlikely to sustain. Sustainable change also requires addressing teacher efficacy-about their skills and preparation to support the student-and the educational environment supports in place. 
The next several sections will consider how to address each level of variable, with the focus first on how to influence changing educator attitudes. Attitudes related to teacher-related variables and education environment-related variables will be addressed in the subsequent sections.

\section{Shifting Educator Attitudes}

Teachers have long been concerned about the individualized time needed to teach students with ID in their classroom, as well as adequate supports and deficiencies in their own skills, training, and support needed to do the job well (J. Campbell et al., 2009). Teacher openness to including students with ID in the general education classroom is related to the severity of the disability (child-related variables) and the teacher's skillset (teacher-related variables) (Avramidis \& Norwich, 2002). While there is a significant body of work on how to support pre-service teachers with education, training, and positive field experiences, the challenge is to effect adaptive change for a wide range of currently practicing teachers (J. Campbell et al., 2009; Forlin \& Chambers, 2011; Kim, 2011; Rainforth, 2000; Van Laarhoven et al., 2007).

To effect adaptive change in the context of and tendency toward deficit ideology, school leaders must address both individual beliefs and prejudices and the structural and systemic barriers in the organization (Valencia, 1997; Gorski, 2011). An example of shifting a systemic barrier is a school leader supporting ongoing professional development and creating a master school schedule that allows time for planning with peers or coaches and creating the conditions to allow co-teaching to occur. Sustaining 
attitudinal change is more complex than changing minds. Attitudes depend on a person's perception of the likely outcome or impact of a situation, with a positive perception resulting in a positive attitude (Sosu \& Rydzewska, 2017). Therefore, teacher attitudes towards inclusion result, at least in part, from their own skill to effect positive outcomes in inclusive settings. Addressing teacher attitudes, then, also requires development of new skills alongside new ideas, both of which can be done with support and point of performance coaching. Peer coaching (with adequate time for co-planning) is one of the most effective ways in which teachers improve inclusive practices in their classrooms; peer coaching can also have a positive impact on attitudinal variables (Nishimura, 2014). Aguilar (2020) described Transformational Coaching as a model that can address "a client's behaviors, beliefs, and ways of being" and surface "the ofteninvisible and often-inequitable systems in which they work" (p. 34). If they are given input into their own learning in the context of a trustful relationship with a coach, teachers can successfully work through the vulnerability in the process of moving towards inclusive practices (Stover et al., 2011).

\section{Attitude and Teacher Support Variables}

The inclusion of students with ID in the general education environment is a complex endeavor. Attitude is a reflection of beliefs and self-efficacy (M. R. Olson et al., 1997). Shifting or influencing educator attitudes toward inclusion of students with ID requires addressing multiple factors simultaneously, including preparation and education for stakeholders regarding the nature of each student's disability; increasing 
understanding of support structures that can make inclusive placements successful; and ongoing professional learning, inservice opportunities, and coaching to build educator capacity (Frattura \& Capper, 2006; McLeskey \& Waldron, 2002; Sari, 2007; Stanovich \& Jordan, 2002). Many teachers in service today were trained in the context of segregated settings (Hall, 2019). Movement toward inclusive educational practices must consider this dynamic.

Positive teacher attitudes toward inclusion are impacted through positive experiences with inclusive classrooms, teacher training and support, resources, and mastery of expertise for inclusive teaching strategies (Avramidis \& Norwich, 2002; Monsen \& Fredrickson, 2004; Scruggs \& Mastropieri, 1996). Effective teachers are at the heart of effective inclusion; achieving that requires that teachers work on the tools they use to practice their craft: pedagogical strategies, curriculum quality, and instructional design. Variables for teacher efficacy, as organized by Loreman's (2007) pillars, are important to making and sustaining teacher attitudinal change away from presuming segregated settings are the default placement for students with ID and toward inclusion.

\section{Teacher-Related Variables}

Meaningful inclusion happens between a teacher and student. Teacher-related variables - such as gender, age, years of experience, grade level, contact with students with disabilities, and personality-all play a role in their attitudes towards inclusion (Avramidis \& Norwich, 2002). While many of these variables cannot be manipulated, it is helpful to think about what teachers truly need professionally in order to create the 
conditions where openness for inclusive practice and positive attitudes can flourish. Loreman's (2007) pillars that consider teacher-related variables are Flexible Curriculum and Pedagogy, Meaningful Reflection, and Training and Resources.

Loreman's Fourth Pillar is Flexible Curriculum and Pedagogy. Curriculum is what is being taught, and pedagogy refers to how the material is to be taught (Loreman, 2007). In Kame'enui and Simmon's (1999) seminal work on inclusive instructional architecture, they set the stage for the next 20 years of expanding ideas on how to effectively teach students with disabilities in the regular classroom. Their focus was on curriculum design and adapting the curriculum for all learners to access the regular classroom. UDL, influenced by the field of disability studies, has evolved how we think about disability and the variability of learners in the context of teaching and learning (Meyer et al., 2014). UDL is an example of a framework that allows and supports teachers to design instruction flexibly and create possibilities from both a pedagogical and curricular standpoint to effectively teach a wider range of students. For example, teachers have been trained for decades within a paradigm that posits that effective teaching means teaching to the average learner, while other systems or experts (e.g., special education, talented and gifted) teach those who do not fit the average profile. The UDL framework and strategies can be a significant support to teachers in order to be effective in an inclusive classroom (Gargiulo \& Metcalf, 2017; Meyer et al., 2014; Spencer, 2011). 
Loreman (2007) suggested that Meaningful Reflection (his Sixth Pillar) is a necessary teacher-related variable that impacts openness for continuous improvement toward inclusive teaching practices. Reflection provides the opportunity for the systematic observation of student results to increase the teacher's use of evidencebased practices. Craig et al. (2019) indicated that for effective learning and implementation of UDL, feedback and reflection are crucial to the process of understanding and delivering on newly learned teaching strategies. Padilla and Tan (2019) argued that teacher reflection is critical to countering ableism. A study of inclusion coaches (Wlodarczyk et al., 2015) found that reflective practice is key to transformative learning for teachers when learning inclusive practices. "Transformation in thinking, beliefs, and practice is a process and often requires a reflective component" (Wlodarczyk et al., 2015, p. 56).

Pillar Seven involves ensuring that teachers receive training and resources for inclusion in order to improve inclusive practice (Loreman, 2007). Specifically, funding and administrative support are necessary for the comprehensive training and professional development necessary to move teachers who have a positive attitude and are open to embracing inclusion to the application of inclusive practices that will sustain improvements in practice and impact positive attitude (Cambridge-Johnson et al., 2014; Boyle et al., 2013). If teaching practices for supporting inclusion are to be implemented, teacher training and professional development are necessary to support effective implementation. Effective professional development can support the improvement of 
teacher practice in teaching students with ID (Hurt, 2007; Thompson, 2012; Strieker et al., 2012; Browder et al., 2012). A rigorous professional development plan that includes content training, active learning, job-embedded collaboration, models and modeling of practices, coaching, feedback, and reflection are all necessary for teachers to effectively implement UDL (Craig et al., 2019). Training and support initiatives must involve local development factoring in prior knowledge, attitudes, and beliefs of teachers involved (Symeonidou \& Phtiaka, 2009). Attitudes, values, and beliefs must be addressed through training, for "practice will never be improved unless teachers are convinced about the significance of inclusion, the dominance of oppressive assumptions about disability that need to be deconstructed and the centrality of their role" (Symeonidou \& Phtiaka, 2009, p. 549).

\section{Educational Environment-Related Variables}

The educational environment must support inclusive practices. Environmental supports [as organized by Loreman's (2007) pillars and affirmed by Avramidis and Norwich (2002)] are necessary for teachers to make and sustain attitudinal change away from ableism and toward inclusion. The tone and encouragement of building leadership can significantly positively influence attitudes toward inclusion. Support can be viewed as physical and human (Avramidis \& Norwich, 2002). Physical supports include resources and tools, assistive technology, and physical space. Human resources can involve expertise from specialists on a consulting basis, classroom assistants to assist with supports, and specialists involved in co-teaching strategies. If a barrier toward inclusive 
education is partly based on the predication of the medical model, which reinforces the assumption that an expert must address impairments within an individual (Symeonidou \& Phtiaka, 2009), placing expertise in the classroom from specialists can change the narrative, while also supporting and building teacher capacity. Experts such as speechlanguage pathologists, school psychologists, school counselors, motor specialists, and learning specialists can all contribute to informing effective learning strategies, accommodations, and modifications that can be refined over time to become highly effective for that student's learning.

The role of related services is a powerful and necessary resource in order to support the inclusive educational environment for students with disabilities (Spooner et al., 2014). Related services are supports necessary for students with disabilities to benefit from special education services. The IDEA requires IEP teams to consider how related services might support students to access the general education curriculum (34 CFR $\S 300.34)$. Examples of related services include speech-language pathology and audiology services, interpreting services, psychological services, physical and occupational therapy services, recreation, counseling services, orientation and mobility services, assessment services, and parent counseling or training. Related services are included on students' individualized education programs, specified as either direct or consultative services. The aforementioned Endrew F. v. Douglas County School District Re-1 (2017) decision specifically addresses the need for the IEP, which includes related services, to be "... reasonably calculated to enable a child to make progress appropriate 
in light of the child's circumstances" (Endrew F., 2017, 69 EDELR 174, p. 2). The expertise of specialized related services providers can support inclusion through direct service, indirect service (effective consultation), and offering their expertise when team problem-solving must happen (Spooner et al., 2014).

A teaching and learning framework that supports inclusive teaching strategies is a critical component of a supportive educational environment (Navarro et al., 2016). UDL is an example of the kind of framework needed to normalize inclusive practice and inform areas for growth in practice. UDL is built upon the assumption that human variability (and difference) is predictable; therefore, educational systems should design instruction to address a wider range of learner needs (Meyer et al., 2014; Rose, 2013). UDL is a framework based on neuroscience that attends to all facets of the learner's experience. The goal within the framework is to develop expert learners. Meyer et al., (2014) defined an expert learner as "one who is purposeful and motivated (the 'why' of learning), resourceful and knowledgeable (the 'what' of learning), and strategic and goal-directed (the 'how' of learning)" (p. 25). Used with fidelity, the UDL framework (Meyer et al., 2014) provides high-quality first instruction that addresses the different parts of the brain in order to develop the expert learner. There are three parts to the framework. The first addresses affective networks (the "why" of learning) that must be addressed by providing multiple means of engagement. Recognition networks (the "what" of learning) are addressed by providing multiple means of representation. Strategic networks (the "how" of learning) must also be addressed, and can be done so 
effectively through multiple means of action and expression. This framework is an example of a necessary environmental support that meets the fourth pillar of Loreman's (2007) work (Spencer, 2011; Meyer et al., 2014; Gargiulo \& Metcalf, 2017).

Assistive technology is significant resource that is important to consider as a support element in the educational environment (Messinger-Willman \& Marino, 2010). The availability of devices and web-based tools have grown as educational service providers and companies continue to develop cutting-edge technologies that allow for improved accessibility. Examples of widely available assistive technology that are webbased include text-to-speech, to assist with reading; speech-to-text, to assist with writing; and word prediction software, to assist with writing. Through assistive technology, reading libraries can be modified to make them accessible for students who need the text at a different level. Assistive technology consideration is particularly complementary to a broader, holistic approach such as UDL in supporting inclusion (Messinger-Willman \& Marino, 2010).

Another key environmental variable, according to Loreman (2007), falls under Pillar Four, Policy and Leadership. Because school districts operate independently, policy plays an important role in outlining improvement priorities for a district. Policy can help define not only priorities but what is meant by inclusive education. According to Ainscow et al. (2000), policy for inclusive education should be:

- Short, containing a view of the future and basic values and principles.

- Stable and unchanging.

- Capable of being internalized and applied to other areas of planning.

- Developed through the engagement of all stakeholders. 
- Clear, despite the diversity of opinion amongst stakeholders.

- Led by the District.

- Supported by a clear government lead.

Policy development involves engagement with the local school board, the elected and representative body of the community for each district. Loreman (2007) argued that community engagement is a key support element in inclusive education. Specifically, school districts can support inclusion through community engagement by bringing parents in as decision-makers in meaningful ways, recognizing parents as their children's first teacher, and seeing parents as advocates for their children. This pillar also suggests that other agencies, such as advocacy and parent education groups, are necessary partners in the work (Spooner et al., 2014).

\section{Leadership, Implementation, and the Complexities of Change in Public Schools}

Inclusion happens through interactions at the student and teacher level, but substantial progress in increasing the effective inclusion of students with ID requires a comprehensive district plan and approach. This need is the focus of this study: if ableist beliefs and attitudes are a barrier to the inclusion of students with ID, what actions and efforts do district leaders attribute to addressing and changing ableist beliefs in the district, and what other efforts do they attribute to increasing effective inclusion in their district?

Effective leadership to administer inclusive policy is necessary at the district and building levels. Leaders must be able to set a vision and direction, understand where to implement changes to remove barriers, keep motivation high, and set the tone for the 
inclusion of all students (Lambrecht et al., 2020). Leaders must be collaborative and supportive of lesson development or design. These leadership characteristics are also referred to as transformational leadership (Lambrecht et al., 2020). Loreman (2007) points out that shared leadership (empowering teachers to become leaders) is not enough of a leadership structure; transformation towards inclusion is also about leading the school community to foster a positive culture that values belonging for each student. With leadership holding such a key role in setting and carrying out policy, engaging the community, and ultimately working toward changing attitudes, this study interviews the leadership of three districts in Oregon to understand actions each district took that the leaders attribute to the district's success increasing inclusion of students with ID. In order to lead complex change, leaders need a framework for implementing innovation like inclusive practices. Implementation science is an organizational theory focused on how innovations, or practices, are taken from research to practice, to ensure that the implementation process accounts for local relevant contextual factors in order to be successful (McKay, 2017). This approach is different from the traditional management of a "roll-out" of a new initiative (National Implementation Research Network [NIRN], 2020). The model focuses on the relevant and dynamic factors that exist in a complex organization, including organizational and human elements. It is a useful framework for analysis and understanding of how innovations have been applied and suggests a pathway forward to address the problem of segregated placements for students with ID. The NIRN synthesized research related to implementation (Fixsen 
et al., 2005); from that, NIRN developed five Active Implementation Frameworks to highlight the critical elements for effective implementation: Usable Interventions, Implementation Stages, Implementation Drivers, Implementation Teams, and Improvement Cycles (NIRN, 2020).

Usable Innovations considers the appropriateness of implementing a desired innovation (SISEP, 2020). Innovations are appropriate to implement if they are teachable, learnable, doable, and readily assessable in practice. Implementation Stages considers a process involving multiple decisions, actions, and corrections to change the structures and conditions necessary to successfully implement and sustain new programs and innovations. Examples are Exploration, Installation, Initial Implementation, and Full Implementation. Implementation Drivers are based on common features that exist among many successfully implemented programs and practices. Implementation Teams actively work together to plan the implementation of innovations (as opposed to the traditional route of dissemination and diffusion). The team approach results in higher-quality implementation. Improvement Cycles include engaging in iterations of Plan, Do, Study, Act as part of an ongoing evaluation cycle (Bryk et al., 2017).

Implementation Drivers (see Figure 4; SISEP, 2020) is a framework that helps illustrate the complexity of the systems responsible for the successful implementation of an innovation or program. It outlines the structural components and activities that make up each driver's contribution to the successful and sustainable implementation of 
innovations and programs (SISEP, 2018). In this study, the Implementation Drivers are used to understand organizational dynamics in order to consider and see aspects of the current system that perpetuate segregated placements for students with ID. Moreover, this framework allows for a methodological approach to the change process as it considers readiness and the myriad of drivers needed to successfully implement change and practices. Implementation drivers (SISEP, 2020) are organized into three categories, each represented by one side of the triangle diagram in Figure 4: Competency Drivers, Organization Drivers, and Leadership Drivers.

\section{Figure 4}

Implementation Drivers

\section{Implementation Drivers}

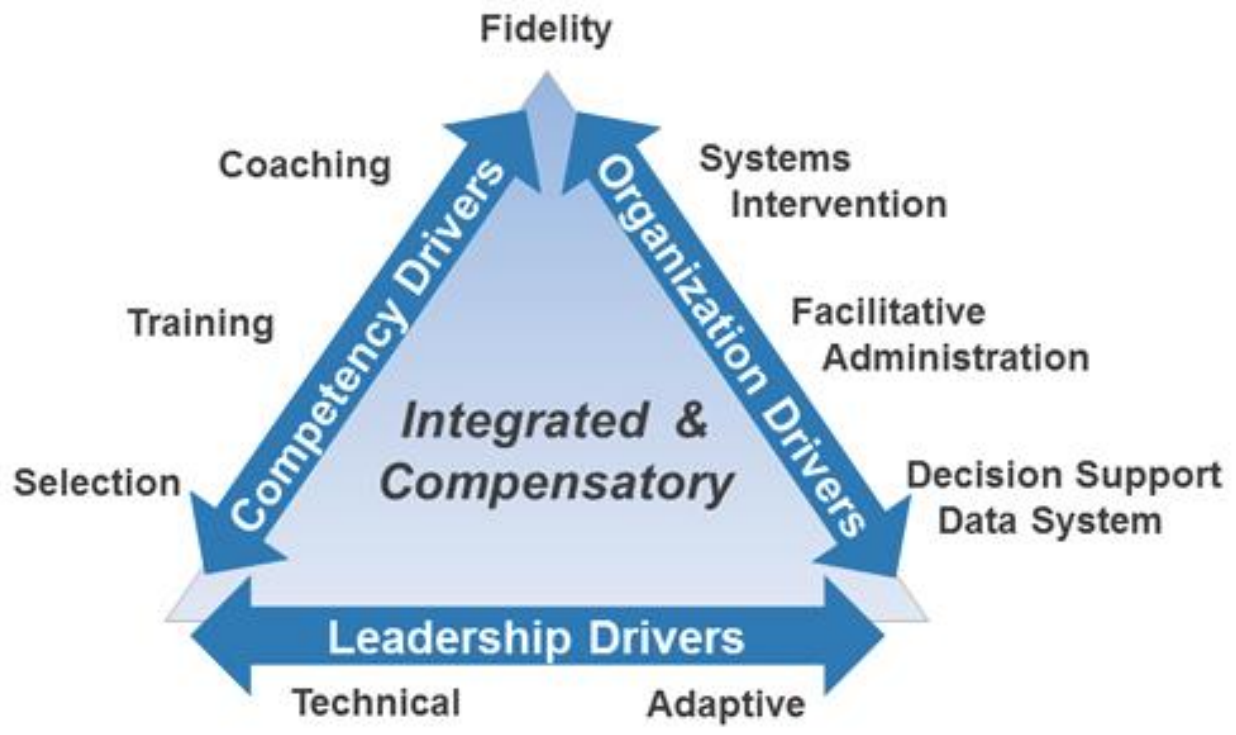

Source: SISEP (2020). 


\section{Competency Drivers}

Competency drivers focus on the skillset of the professionals involved through addressing teacher-related variables necessary, such as effective professional development (Craig et al., 2019), to sustain inclusive practices. The drivers include hiring and selection of staff, training practitioners, support through coaching, and fidelity measures. These drivers all impact positive attitudes by addressing teacher-related variables (Avramidis \& Norwich, 2002) aligned with Loreman's (2007) Pillars Six and Seven: Meaningful Reflection and Training and Resources. When looking at Competency Drivers (Figure 4; SISEP, 2020), considering the selection of staff, training, coaching, and fidelity measures, a question emerges with this problem of practice: how are educators prepared to do the work of meaningful inclusive practices in the general education environment? Another barrier having to do with competency is ensuring sufficient time for coaching, collaboration, and support needed to address the root causes of segregation determined through analysis with local practitioners.

\section{Organization Drivers}

At the heart of systems change, organization drivers connect to and impact teacher-related variables and educational environment-related variables (Avramidis \& Norwich, 2002). Inclusive practices happen within the organizational context, it follows that organization drivers are the pathway to influence Loreman's (2007) Pillars of Curriculum and Pedagogy, Training and Resources, and School and Classroom Processes. Considering Organization Drivers (Figure 4), systems intervention considers district-level 
commitment, policies and procedures, and systems variables. Organization drivers at the district level compel analysis of system interventions (i.e., what innovations are already in place that help or hinder), data systems (i.e., how to measure incremental success), and facilitative administration (i.e., measuring and understanding how open leadership is to a certain innovation) (NIRN, 2020; SISEP, 2020).

\section{Leadership Drivers}

Leadership drivers are necessary considerations for leaders to address childrelated, teacher-related, and educational environment-related variables (Avramidis \& Norwich, 2002). They help frame the types of leadership challenges that are barriers to Loreman's (2007) pillars of Policy and Leadership, the Community, and School \& Classroom Processes. Leadership drivers are an assessment of the barriers in terms of whether they present an adaptive or technical challenge. Technical challenges are simpler in nature and have clear, linear solutions. Teams can agree on both the scope of the problem and a solution that will address the problem. They can be managed by fewer individuals close to the problem and with the right authority. Conversely, adaptive problems are unable to be resolved through traditional practices of leadership. They involve deep shifts in beliefs, values, perspectives, and typically compelling growthpersonal and professional-in order to effect a shift in practice (Heifetz et al., 2009).

\section{Synthesis}

This study investigates the problem of disproportionate segregated school placements for K-12 students with ID. The theoretical frameworks used in this analysis 
are ableism; Seven Pillars of Support for Inclusive education (Loreman, 2007); and Variables on Addressing Attitudinal Barriers (Child-Related, Teacher-Related and Educational Environment-Related) (Avramidis \& Norwich, 2002). These theoretical frames allowed this study to surface an explanation of the attitudinal barriers that keep segregated placements for students with ID entrenched (linking ableism and deficit ideology as contributors to the problem). They also provide a framework for leaders seeking to improve inclusive practices.

This study identifies schools and school districts that have demonstrated a reduction in segregated placements in their schools by improving inclusive practices. These systems, from a methodology standpoint, are bounded systems. Bounded systems are defined as a system with its own contextual variables (Yin, 2018), where the application of a school district is a bounded system in that it is an organization with local control of its own policies, practices and procedures for going about the business of educating students within its boundaries. The study's theoretical frames support the analysis of data collected by allowing an examination of the relationship between leadership practices and the theories presented. The analysis in this study contributes an examination of local leadership practices related to inclusive practices for students with ID.

The review of the literature finds overwhelming evidence of the need for schools to become more inclusive in their practices, in particular for students experiencing ID; affirms that segregated placements for students experiencing ID are disproportionate 
when comparing other students identified with other disability categories; and confirms that the problem is persistent over time. Moreover, the literature review revealed that the dynamics and influence of unexamined ableism are connected and contribute to this problem. Themes that emerged in this literature review surface the tension between the benefit of inclusive education for students with ID and the complexity of reform in public education to engage in practices that are not required by law (under the IDEA, districts must provide a continuum of alternative placements, including special classes and schools, which suggests that the law requires segregation, at least for some students, rather than inclusion). As cases like Endrew F. v. Douglas County School District Re-1 (2017), and Lane v. Brown (2016) apply pressure to districts to improve outcomes (raise expectations and prepare students with disabilities for life after graduation), school administrators are working to balance these priorities and achieve their mission. This literature review also highlighted the complexity of change management in schools seeking to implement evidence-based practices in order to sustain improvements. The addition of political dynamics in school districts, where governance falls to elected community members who set policy, among other tension points (e.g., finite resource allocation to public education), makes it clear why this problem is so persistent and challenging to resolve.

\section{Critique}

There are volumes of scholarship examining the dynamics of inclusive education for students experiencing disability; from the efficacy and outcomes, to the practices of 
inclusion, to methodology in leadership and classroom practices. The literature outlines the benefits of inclusive education (although there are a few voices critical of this notion), and longitudinal studies suggest that for students with ID, outcomes (i.e., readiness for employment, further training, or university study) are predicted to be improved (Test et al., 2009; Test et al., 2016). Many frameworks and theories guide and lead those engaged in this work. Outlined in the literature review are organizational change theories and the dynamics involved in order to implement sustainable improvement over time in order to surface the tension between the research and actual implementation of inclusive practices. The gap in the literature is an examination of local leadership practices that have led to a reduction of segregated school placements and increased access to rigorous general education curriculum predicted to improve outcomes for students with ID. In other words, given the complexities and challenges involved in public education reform, there is a need to understand how leaders have led their districts toward improved metrics. In particular, for Oregon schools and districts, state school funding practices combined with special education maintenance of effort requirements increase the difficulty of securing a firm, ongoing investment of the general fund resources necessary to address the persistent segregation of students with ID. This study, examining Oregon districts, addresses the aforementioned gap by comparing leadership practices to selected theoretical propositions and highlighting other nuanced leadership practices that are important to consider when working to solve this problem of practice. 
The literature contains opposing viewpoints, with some dissenting works that argue the value and benefit of separate placements for students experiencing disability. There are opposing views that do not necessarily disagree with inclusive practices for students with ID, but highlight where implementation is poorly led, resulting in an experience that is not positive for teachers or students. For example, Gilmour (2018) argued that the research that demonstrates improved outcomes for students with ID is methodologically flawed and has not considered the impact on non-disabled peers and teachers. Some critics argue that being in the general education setting does not mean they are making progress toward goals simply because they are in the space (Gilmour, 2018). Gilmour argued that teacher turnover has dramatically increased in this current age of increasing inclusive placements for students with disabilities. She further points out that special education is a service, not a place, even a less restrictive placement, and that policymakers should not use placement metrics as a gauge for academic success.

The critique of inclusion for students with disabilities center on general education teachers who are not sufficiently prepared to create meaningful access to the general education curriculum for students with disabilities. High-quality implementation of inclusive practices is at the center of how a change in public education can improve this problem of practice. This study is necessary to understand the leadership components needed to implement and sustain improvements in Oregon, given local context and variables. 


\section{Methodological Literature}

Educational research examines problems of practice in context, works toward an understanding of the problem, and proposes solutions to improve student learning (Hamilton et al., 2013). The problem posed in this study, like many problems in education, is multi-faceted and complex. In order to understand the challenges underlying the widespread segregation of students with ID and identify potential solutions, the phenomena must be studied by looking at schools and the districts where they operate. Because school districts operate and govern themselves, local control and decision-making factor into how this problem is studied. The choice to look to exemplar districts, where those local decisions and processes are manifested, suggested the use of a qualitative research design, with data from interviews of district leaders who have been successful in increasing the inclusion of students with ID.

From a theoretical perspective of constructivism, reality is constructed and informed by knowledge, experience, and belief. For example, the current laws written to assure educational equality for students with disabilities are predicated on a medical model that purports that disability is an impairment that impacts the ability to access learning. This predication contributes to educator beliefs that students with disabilities should be fixed, by an expert, and somewhere else. This construction, combined with a deficit ideology, are barriers to positive attitudes toward educating children with ID in the general education setting. Disabilities Studies suggests that the impairment of a child with a disability is not to be fixed but, rather, the impact of the impairment is to be 
ameliorated through skill-building and accommodation in the general education setting (Watson et al., 2012). Additionally, Altermark (2014) suggests that how we define ID has changed and should be reconsidered. Because educators construct their reality and practice based on their training (knowledge), experiences, and beliefs, in order to understand how local districts are achieving progress in addressing the problem of segregated placements, a qualitative interview was the best option to understand multiple perspectives (Hamilton et al., 2013).

Because the questions that drove this study were process questions seeking to understand the processes by which district leaders have increased inclusion rates in their district rather than variance questions, qualitative inquiry was selected (Maxwell, 2013). A case study is an investigation of phenomena within the context of a bounded system (Yin, 2018). A multiple-case study allowed an inquiry to take place to understand what each district's (case) leadership was doing, how they viewed the problem in their context, and what, in their viewpoint, contributed to their success in improving inclusive practices.

\section{Summary of the Research Literature and Application to the Study}

The research literature outlined in this study affirms that there is a longstanding history in the United States of a separate education system for students experiencing ID. The IDEA compels districts to educate in the LRE, and to offer a range and continuum of alternative placements. While inclusion for all students with disabilities is not the goal of the IDEA, education for students with an ID remains persistently disproportionately 
segregated. A contributor to this problem is the effect of ableism and deficit ideology that impacts decision-making. In other words, educator attitudes indicate their openness to inclusive education opportunities, which impacts the effectiveness of inclusive education. There are child-related, teacher-related, and educational environment-related variables that must be considered in order to support the people in an education system to create a positive condition for inclusive education. Loreman's (2007) Seven Pillars of Support for Inclusive Education offers a framework for understanding the interacting system complexities critical to reducing segregated placements and improving inclusive practices in schools. In order to answer the research questions raised in this study, an explanatory, multiple-case study is used to investigate the phenomenon of how Oregon districts are increasing inclusive education in their schools. 


\section{Chapter 3 Methods}

Students with ID are disproportionately segregated from their peers without disabilities in where/how they receive their education. Research on inclusive education has very few studies that examine how districts improve or move toward inclusive practice (Jackson et al., 2008). The research goal of this study is to understand how educational leaders in Oregon move away from segregated placements for students with ID and work toward increased inclusive practices in the general education classroom. The focus of this study is to identify and investigate districts that have demonstrated a move away from segregated placements and move toward inclusive practices for students with ID.

\section{Research Methods}

Qualitative research is appropriate when the research questions are process questions, as opposed to variance questions. School districts in Oregon are governed by a school board-elected local citizens-maintaining local control. While each district must adhere to standards set forth in the administrative rules established by the state board of education, each local school district is responsible for determining the policies, procedures, and practices by which they will ensure compliance to these requirements. School districts are clearly identified learning organizations that are self-governing, with geographical boundaries identifying its clientele. They choose their strategic priorities and policies are decided upon by elected officials, school board members $\backslash$. Those policies are then carried out by each district's administrative staff. Each of Oregon's 197 
school districts function differently based on this proposition in light of their local context. This study sought to understand what leaders in independent districts attribute to recent increases in inclusive placements and practices for students with ID.

A case study is a qualitative method that will allow a study of phenomena within their context in a bounded system (Yin, 2018). For this study, the phenomenon to be studied were leadership actions (thinking, planning, actions) that led to improved inclusion for students with ID. A multiple-case study design allowed examination of the phenomena in different bounded systems in order to draw comparisons and answer the research questions. A multiple-case study was selected as districts may take novel approaches to the problem, given each district's different set of unique challenges. Multiple-case study addressed external validity as well as offered leadership practitioners insight as to how to address some of the unique challenges associated with leading for inclusion.

\section{Participants}

Multiple districts were selected in order to inform answers to the research questions in this study. Selection criteria were established to ensure appropriate selection of districts that were improving in their inclusive practices for students with ID. District selection included input from 19 regional special education directors and nine Oregon Department of Education special education specialists. These experts provided input through a survey (see Appendices E and F) as to which districts in their area have improved inclusion for students with ID. Data were also obtained from the Oregon 
Department of Education through a public records request to confirm input from the survey through a review of the annual special education census for the state of Oregon. The data set includes the number and percent of students with ID and their Federal Placement (Indicator B5) category, for all 197 districts in Oregon. Districts were selected based on this input and data (see Table 1).

Three districts in Oregon, with varying sizes and geography, were selected to increase validity and generalizability of the findings. Once three districts were selected, initial contact was made with each district superintendent to obtain permission to participate in the study. District selection utilized three sources of data, all of which had limitations (e.g., surveys were voluntary and had less than $50 \%$ participation). The data sources for district identification included:

- Survey responses from 3 of 19 Regional Special Education Directors (sent March 2021)

- Survey responses from 5 of 9 Oregon Department of Education special education specialists (sent April 2021)

- Federal Placement (Indicator B5) Data Set indicating placements by number and percentage of students with a primary disability category of Intellectual Disability for the 17-18, 18-19, and 19-20 school year. (Obtained September 2020)

Starting with the surveys, which reflect perception data of two sets of professionals whose role it is to support/monitor district operations from a regional or statewide perspective, districts emerged on two lists: those identified by regional special education directors and those identified by state specialists. Three districts were identified in both lists. Data from districts identified by both sets of experts were 
analyzed to confirm that segregated placements were decreasing and placement in the general education setting for students with ID was increasing. Figure 5-7 illustrate the Federal Placement (Indicator B5) data trends over 3 years for the three selected districts.

\section{Figure 5}

District 1, Federal Placement (Indicator B5) Placement for Students with ID in Gen Ed Over Time

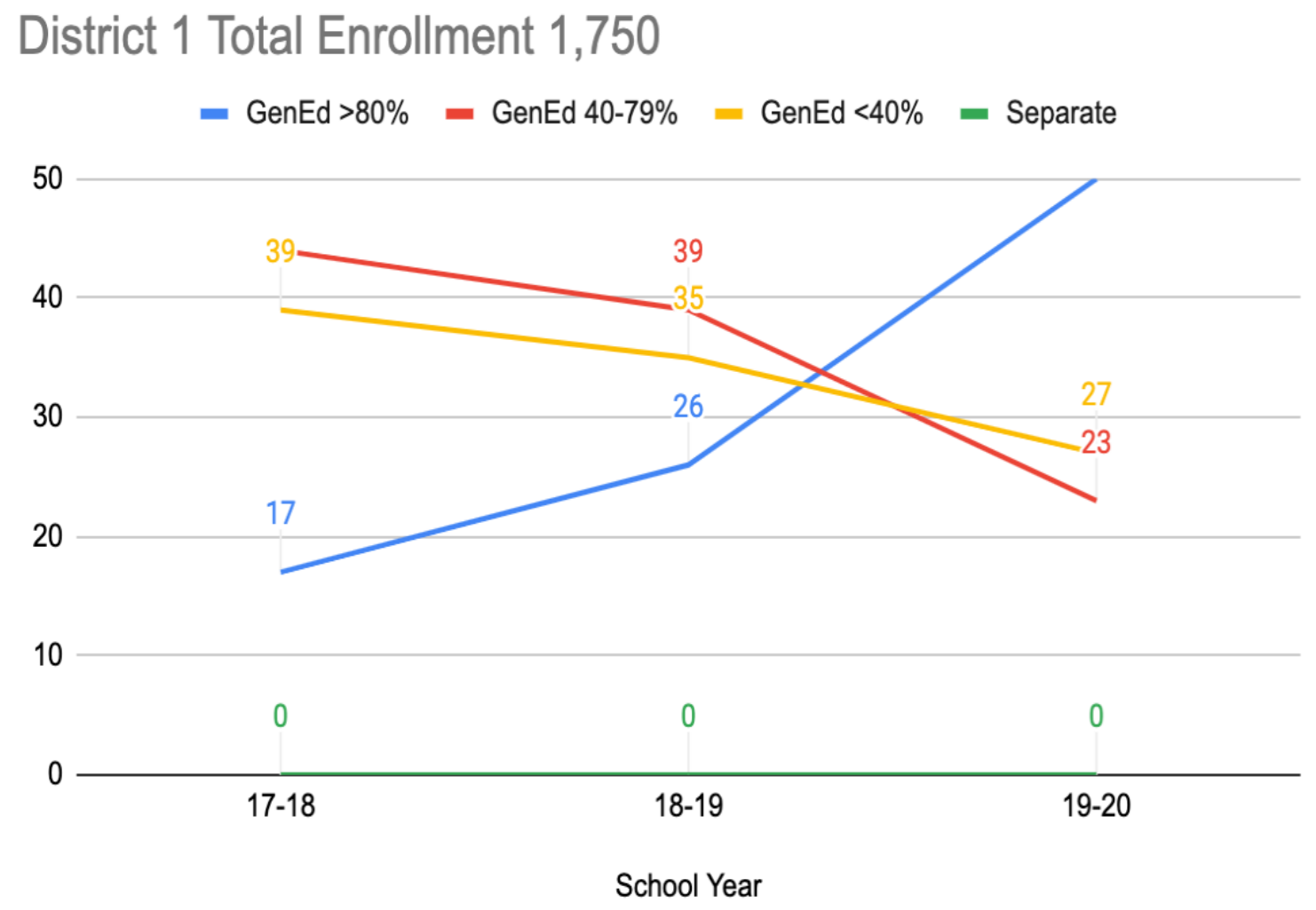




\section{Figure 6}

District 2, Federal Placement (Indicator B5) Placement for Students with ID in Gen Ed Over Time

District 2 Total Enrollment 9,802

$$
\text { - GenEd }>80 \% \text { - GenEd } 40-79 \% \text { - GenEd }<40 \%
$$

50

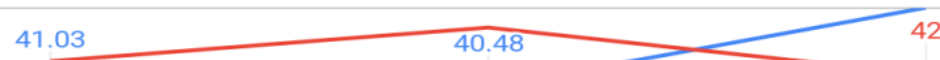

40

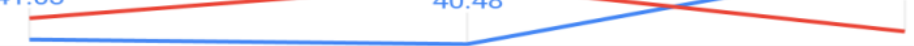

30

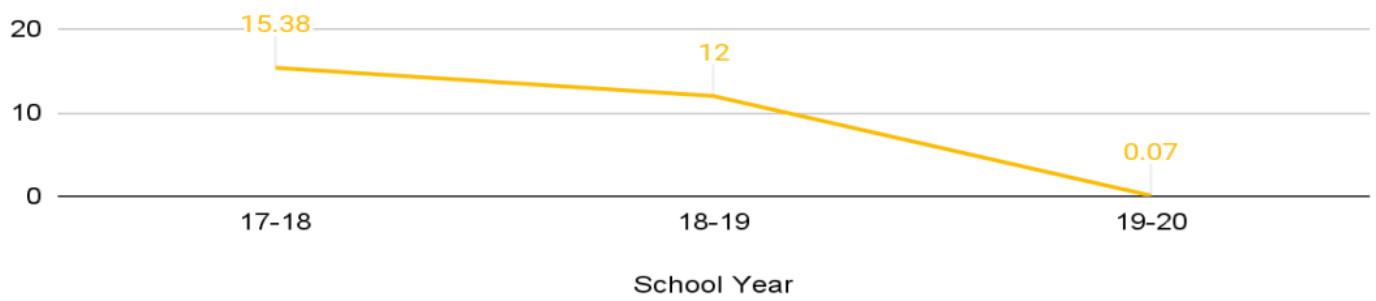

\section{Figure 7}

District 3, Federal Placement (Indicator B5) Placement for Students With ID in Gen Ed Over Time

District 3 Total Enrollment 20,269

$$
\text { - GenEd }>80 \% \text { - GenEd } 40-79 \% \text { - GenEd }<40 \%
$$

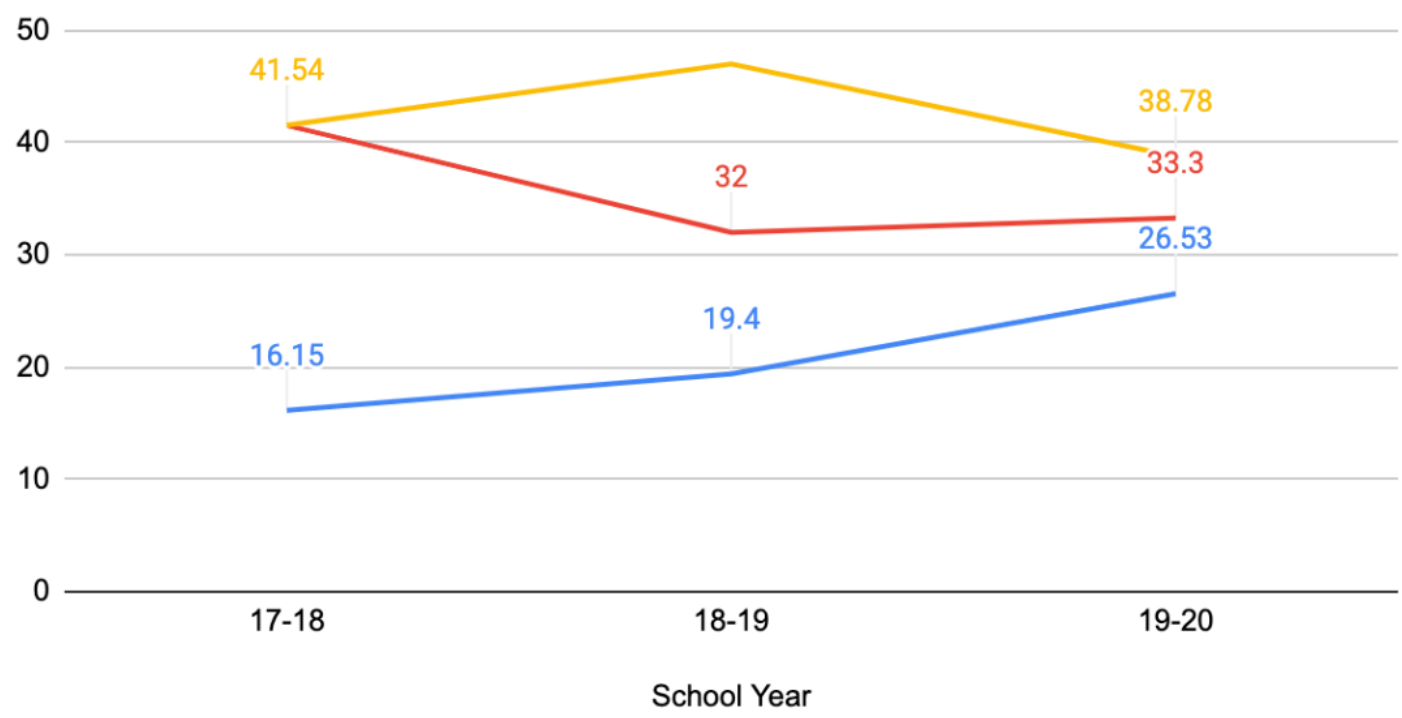


Data analysis revealed that all three selected districts increased the percentage of inclusive student placements (participating for more than $80 \%$ of the time with peers without disabilities). Conversely, all three districts show a reduction in placements where students are placed in the general education setting less than $40 \%$ of the school day. This data confirm that placement for students with ID is moving away from segregated settings and towards inclusive education in the general education setting within each of the selected districts. These three districts varied in both their geography and student population.

Participants in this study are key leaders and decision-makers in each district. Administrators who participated in the study included the administrator over curriculum and instruction, the district special education administrator, and a principal. These participants were selected as they each lead a different segment of their organization, all of which contribute to improved inclusive educational practices at a school. The special education administrator is responsible for enabling the provision of a free appropriate public education (FAPE), which is the legal obligation to for all students who experience disability eligible for support under the IDEA. Such requirements are driven by the IDEA at the federal level; by Oregon Revised Statutes and Oregon Administrative Rules at the state level; and by policy, procedures, and practices at the district and school levels. Special education directors are responsible for the delivery of services and processes where decisions about placement are informed, considered, and made at the individual student level. Curriculum and instruction leadership perspective was sought 
because inclusive education happens within the structure of the general education learning environment. The general education system is built on teaching and learning frameworks led by this district-level leader. Their perspective, combined with the district continuous improvement process, brought information and understanding to the case study about how the districts and schools are supporting teachers with the framework and evidence-based practices that will allow students with ID access to the rigorous content offered in the general education classroom. Principals of selected schools were sought as the single most influential leader when it comes to the quality of instruction, in particular, for students with disabilities (Bateman \& Bateman, 2014; Riehl, 2009; Wells, 2016). Their instructional leadership is key for leading increasing inclusive teaching and learning in their school.

\section{Procedures}

An application to the Institutional Review Board (IRB) was submitted, and approved as an exception to IRB; the study was approved to proceed as planned on March 1, 2021. Following approval for the study, an introductory email communication was sent to selected district superintendents. Once permission from district superintendents was obtained to participate in the study, superintendents recommended leaders from their district to participate. The recommended participants were sent an introductory email explaining the purpose of the study and inviting their voluntary participation. Once the selected participant responded and agreed to participate, interviews were scheduled and artifacts were requested. Because the 
interviews were conducted using teleconference technology (Zoom) and recorded (given restrictions of a study during a pandemic), consent was affirmed by each participant on the record, as approved by the IRB.

Interviews were scheduled and conducted via teleconference by the researcher. The recorded interviews and transcripts reside in a secure, password protected, cloudbased repository for access, safety, and security. An interview protocol (Appendix C) was followed, with the questions therein. Initial questions were the same across participant leaders. Because the interview was semi-structured, research methods allowed flexibility to expand and probe different areas of expertise and perspective given participants' varying roles and responses.

Interviewer bias refers to factors that influence the collection of the data because of aspects related to the interviewer (Frey, 2018). This bias can include positionality, perception of the interviewer's identity, and professional background, especially because these characteristics are related to the interview topic in the current instance. This author's positionality as a sitting district-level special education director impacted the data collected. Participants were generally forthcoming, but may also have been reluctant to share perceived setbacks for fear of judgment from a colleague.

Reflexivity is the notion of embracing interviewer bias in a way that works toward an understanding of the dynamic rather than trying to eliminate the bias (Bourgeault et al., 2010). Reflexivity tenets are about the attention paid to how the phenomena are filtered through the lens of the researcher, recognizing both that the 
study impacts the researcher and that the researcher impacts the study setting. In order to address this bias, this author was cognizant of the impact of these dynamics during the interviews, built rapport with each participant, and balanced rapport and professional collegiality by maintaining a professionally distanced stance. Bourgeault et al. (2018) suggested that the practice of reflexivity brings transparency to the process. In other words, authentic rapport with the interviewees and transparency around the process are appropriate measures to mitigate the impact of researcher bias. Brantlinger et al. (2016) suggested that bias can also be mitigated through triangulation. In the current study, triangulation was achieved through the aforementioned reflexivity transparency, member checks, and peer debriefing through analysis.

Documents were requested from each district in order to collect artifacts to examine, supporting the multiple case study design with more points of data. The artifacts reflected strategic priorities, planning, thinking, and implementation of innovations to improve inclusive practices in the district. The requested documents included:

- District Continuous Improvement Plan (required by ESSA and Oregon's Student Investment Account planning);

- Building Continuous Improvement Plan (for schools selected for principal interviews);

- Teaching and Learning Frameworks and professional development scope and sequence;

- Procedure manual with guidance for making placement decisions for students with disabilities, and;

- Any additional artifacts suggested by participants from each school district. 


\section{Instruments/Measures}

The goal of the study is to look to three Oregon districts to understand leader thinking, planning, and actions that have contributed to improving inclusive education for students with ID. In order to understand thinking, planning, and actions, interviews of these leaders were selected as the main data collection strategy. This study utilized a single, semi-structured interview with each of the participants (see Appendix C). The interview was designed to let participants speak frankly about their views and perceptions. Interview questions started by asking participants to review and respond to their district's Federal Placement (Indicator B5) data, framing the interview in each district's local context. Questions move into open-ended questions such as, "how is inclusive education working from your perspective" and "What are the key organizational factors from your perspective that allow students with ID to learn in the general education settings"? The questions are designed to ask each leader to share information from their perspective. Interview questions are meant to be limited, broad, and open. Interview questions were drawn and informed by the three theoretical frameworks used to explore the topics. Questions about systems, supports and leadership activities were informed by Loreman's (2007) pillars, questions about attitudinal barriers were informed by Disability Studies and Avramidis and Norwich (2002). Implementation Drivers (see Figure 4; SISEP, 2020) were used to inform interview questions toward the perspective of district leaders who have been involved in implementing change efforts that have led to improved inclusive practices. 


\section{Role of the Researcher}

In qualitative research, the researcher is the primary instrument of inquiry (Maxwell, 2013; Merriam \& Tisdell, 2016). Bias in qualitative research has evolved in social science research as something to embrace, rather than eliminate (Maxwell, 2013). Thus, the researcher's training, background, and positionality are important considerations in the role of the researcher for this study. This researcher is currently employed in an Oregon school district as a special education director. Attempts to move toward inclusive education within his district have proven to be challenging, thus building interest in investigating this problem of practice. Prior to being a special education director, this author was a school psychologist and behavior specialist for 8 years and has served as an administrator for 10 years.

This author's background as a school psychologist and assessor of students experiencing ID impacts how this problem is viewed from an assessor's and district administrator's point of view. Training to become a school psychologist involves understanding the limitations of the instruments used to evaluate and identify a student for services due to experiencing an ID. This process and practice are rooted in the aforementioned notion that there is, in fact, an average human intelligence that can be quantified. It has taken years of evolving an understanding of how limited these instruments are in terms of their predictive validity of human potential for school achievement and success as an adult. The assessment instruments this researcher was trained to use have the potential to contribute to the problem of segregation, 
depending on interpretation and meaning derived. While they can be helpful in guiding teams on how to best intervene, if the limitations are not abundantly part of the discussion and interpretation, intelligence and adaptive assessments can contribute to the notion of ableism and deficit ideology. This researcher has been significantly influenced by scholarship from the field of Disability Studies questioning the value of these instruments; their use in routinely making segregated placement decisions for students with ID has exacerbated the impact of ableism in our school systems and harmed all of us.

As a district-level administrator, this researcher has an evolving point of view seeing how the disability label impacts IEP team considerations related to placement. When this researcher was practicing as a school psychologist, it made perfect sense that once a student was eligible for services as a student who met criteria for ID, a segregated placement could "provide instruction at their level" (often the rationale). In hindsight, this thinking is ideologically deficit-based, and may not afford sufficient access to the general curriculum. This historical positionality in this researcher's background informs a bias contending that cognitive and adaptive assessment tools are limited; labels can be limiting; and labels, in fact, can contribute to ableism and segregation. To address this bias, the interview questions were designed to be open and not leaning toward a particular viewpoint. 


\section{Trustworthiness}

Validity of a study investigating a phenomena as complex as placement for students with ID is an important area to address (Trainor \& Graue, 2014). To address construct validity, which is about the correct operational measures for the phenomena being studied, this study identified the bounded systems to be studied (school districts) and used multiple sources of information to examine the phenomena. The study interviewed the same leadership roles in each of the systems and conducted analysis of published artifacts outlining district priorities, policies and procedures germane to the improvement of inclusive practice. Data such as LRE/ B5 placement data for each bounded system to affirm improvement efforts supports addressing construct validity. Construct validity is addressed in this study by employing multiple sources of evidence in data collection and in conducting two rounds of member checks, after the interview and a review of the draft copy of the study by interview participants (Yin, 2018).

Internal validity is important in an explanatory case study because the explanation is causal in nature and inferred between leader thinking, planning and actions and an improvement in inclusive placements for students with ID. The threats to internal validity need to address rival explanations and attributions to the improvement. Addressing internal validity happens in the analysis portion of the study. The analysis of this study utilized pattern matching, explanation and interpretation and used logic models to describe the phenomena being studied (Saldaña, 2021; Yin, 2018). In the analysis phase, pattern matching is a process embedded in the coding strategy in that 
the theoretical propositions were coded alongside the new themes (components) that emerged in the rounds of coding. These comparisons allowed for rival explanations to be addressed de facto. Triangulation of sources and data during analysis and evaluating major findings that emerged.

\section{Data Collection and Analysis}

Data collection and analysis in this multiple-case study, supported by Yin (2018), was based on theoretical propositions that inform data collection and analytic priorities. In this case, the multiple-case study was explanatory in nature. It sought to understand how and why districts are finding success including students with ID in the general education classroom through examining the thinking, planning, and actions taken by its leadership. Theoretical propositions in this study are drawn from Loreman's (2007) Seven Pillars, and Avramidis and Norwich's (2002) Variables on Addressing Attitudinal Barriers. These theoretical propositions serve as a comparative framework to examine leaders' responses to the interview questions and codify what they describe as influential factors that are key to improving the problem of practice explored in this study.

The analytical techniques used were a combination of Pattern Matching (Yin, 2018) and Pattern Coding (Saldaña, 2021). Pattern matching is about finding patterns or themes in the interview data and artifacts for the district and comparing the emerging patterns with the assumed theoretical propositions (Yin, 2018). Pattern Coding is an explanatory method of coding used in order to identify categories and themes (Saldaña, 
2021). The analysis supported answering the research questions, from multiple

perspectives, from three different bounded and defined systems. The process steps:

1. Interview one participant, transcribe, complete a summary following each interview and share with the participant in order to conduct member check.

2. Before each interview, review subsequent summaries to refresh emergent themes and categories.

3. After all interviews were complete, member checks were conducted by sending an interview summary to each participant for review and feedback (Yin, 2018).

4. Transfer transcriptions of each interview to media files in qualitative data analysis software, Dedoose.

5. Transfer artifacts to media files in Dedoose.

6. Codes in Dedoose were established using Loreman's (2007) Pillars, and Avramidis and Norwich's (2002) variables.

7. Round one of coding included reviewing transcripts and artifacts for relevant excerpts and information in artifacts that supported or described a system, a process, or a practice that contributed to improving inclusive practices (Saldaña, 2021). Those excerpts were coded or categorized and linked to the established codes and as systems, processes, and practices (component) were described by more than one participant or emphasized as important, a new code was established to describe and define that component.

8. Round two of coding involved a review of all excerpts highlighted, once all component codes were established, and linked all excerpts to the various codes.

9. Research Question 1 was addressed by comparing the components (systems, processes, and practices) in each district to look for similarities. This was conducted by separating each districts' data and comparing the districts by the frequency count for each code in order to answer research question one, about similarities and differences between systems processes put in place to improve inclusive practices. The frequency count is a way to determine which components leaders described the most given the same set of interview questions. The advantage of this analysis strategy is to highlight the strength of the particular component compared to other components 
discussed. The risk is that other important, and critical, components, were not illustrated as a major finding- and thereby could be concluded that it is not a necessary element.

10. Compared the frequency counts of components between districts that were a higher count (a component was coded by one district more than four times) and identify those components (Yin, 2018).

11. Compared the Frequency counts of components between districts that were high (a component was described by a district more than 10 times and described less than four times by other districts), and identify those differences.

12. Research Question 2 explores the relationship between Loreman's (2007) Pillars and what leaders describe (components) contribute to improved inclusive practices. This analysis examined co-occurring codes for each pillar and each component. In other words, under each of Loreman's pillars, how many times an excerpt from the interview is coded under that pillar indicates the strength of the relationship. This analysis compares leadership practices with Loreman's theoretical framework of inclusive practice in order to answer the question.

1. Build a filter in Dedoose to analyze the data set by isolating a Pillar (Loreman, 2007).

2. Conducted co-occurrence analysis through the lens of each of Loreman's Pillars, identifying which components were coded more than 10 times under each Pillar (Saldaña, 2021).

3. Graphical analysis highlighted components under each Pillar that were described as important (Saldaña, 2021).

13. Research Question 3 was addressed by analyzing codes linked to excerpts describing attitudinal variables. This question allowed the examination of how leaders across different districts describe their own attitude and educator attitude about inclusion and efforts to address attitudinal barriers. This analysis first started through the lens of Avramidis and Norwich's (2002) framework by separating each district's data using code application frequency counts.

1. Build a filter in Dedoose that allows for separating each district's data set. 
2. Run analysis of code application to review patterns in which components (in each district separately) were endorsed as important to address attitudinal barriers and applied under child-related variables, educational environment-related variables, and teacher-related variables. 


\section{Chapter 4 Results}

Students with ID are segregated from their neurotypical peers in where and how they receive their education. This problem is a critical equity concern for all learners, as inclusive placements benefit children with and without disabilities (SWIFT, 2017). The IDEA compels schools and districts to offer a continuum of alternative placements, including segregated settings when education in the general education setting, even with supplementary aids and services, does not confer meaningful benefit. However, trends in the Oregon Placement Data (see Figure 1) affirm that, for students with ID, the number of students in segregated settings is disproportionately higher when compared to other disability types. This problem persists at a local, state, and national level, in part due to the deficit perception of disability (i.e., under a medical model of disability, they are impaired), historical practices of segregating people with ID, lagging inclusive instructional practices, and unexamined ableist views that limit expectations of the capacity of people with ID. While one could focus on criticizing schools that are disproportionately segregating students with ID, this study examined districts in Oregon where schools have made progress toward inclusive education for students with ID in order to understand how leaders went about improving their systems. This study highlights the components (systems, processes, and practices) related to educating students with ID that presume their competence, challenge them academically, and compel school staff to engage in the discourse necessary to enable schools to remove barriers preventing their access to high-quality, inclusive education. 
Improving inclusive practices for students with ID addresses a systemic and dynamic tension between two seemingly opposing forces. On one hand, our federal and state laws compel districts to offer a range of placement options, including segregated settings, and require IEP teams to place students in these segregated settings when "education in regular classes with the use of supplementary aids and services cannot be achieved satisfactorily" (34 CFR § 300.114). On the other hand, statute (e.g., the 2004 reauthorization of IDEA as the Individuals with Disabilities Education Improvement Act); policy (e.g., revisions to IDEA's significant disproportionality regulations in 2016 at 34 CFR § 300.646-647); and legal precedent (e.g., Endrew F. v. Douglas County School District Re-1, 2017; Lane v. Brown, 2016) have all charged schools with improving outcomes for students and adults receiving special education, including those with ID. The preponderance of available research over the past 30 years reveals that inclusive practices support improved outcomes (Sailor, 2016; SWIFT, 2017). It is this dynamic tension that is of interest to the researcher: IEP teams do not have to include and may have to segregate; yet, $\mathrm{K}-12$ systems must improve outcomes for students with ID which, research suggests, is done through increasing high-quality inclusive practices. However, research on inclusive education for students with ID has very few studies that examine how school districts transition from segregated settings to inclusive practices (Jackson et al., 2008).

This study identified three districts in Oregon making improvements in their inclusive practices for students with ID. These districts varied in size and geographical 
location. The study revealed some interesting distinctions among the districts. The districts will be referred to as districts 1, 2, and 3 for reference purposes and will only be described by approximate district size and geography. District 1 has a total enrollment between 1,500 and 2,000 students and is located in a rural part of the state. District 2 is more populated, within a metropolitan area, and serves about 10,000 students total. District 3 is the largest of the participating districts, with over 20,000 total enrolled students, and is considered to be located in a suburban setting.

\section{Analysis of Data}

Primary data for this study were collected in the form of semi-structured interviews with participants from selected districts. Those interviews were then transcribed to support analysis. In addition to the interviews, artifacts were collected from each district that highlighted policies, procedures, and assessment tools, or otherwise guided and directed the work of their respective organizations toward improving inclusive practices to educate students with ID. Data analysis consisted of rounds of coding interview transcripts and district artifacts (Saldaña, 2021; Yin, 2018). The theoretical propositions that act as the foundation for this analysis are drawn from disability studies (Baglieri, Bejoian et al., 2011; Baglieri, Valle et al., 2011; Davis, 2017), ableism (e.g., Hutchen \& Wolbring, 2012); Loreman's (2007) Seven Pillars of Support for Inclusive Education; and Avramidis and Norwich's (2002) Variables on Addressing Attitudinal Barriers (Child-Related, Teacher-Related, and Educational EnvironmentRelated). 
Data analysis began with identifying relevant excerpts from the raw data sources (i.e., interview transcripts and artifacts) and coding those excerpts to categories. Though coding categories were initially drawn from the theoretical frameworks, emerging categories were also used to capture nuanced differences in how districts and schools approached improvement toward inclusive practices. The next round of analysis explored the frequency of components (systems, processes, and practices) by using the software tool, Dedoose, that surfaced the commonalities and differences that administrators identified as promoting the inclusion of students with ID in general education settings. The first two rounds of analysis addressed the first research question: What are the commonalities and differences in components (systems, processes, and practices) that administrators identify to promote the inclusion of students with ID in the general education classroom?

The next step utilized Pattern Coding (Saldaña, 2021) in order to develop themes out of the categories that could inform meaningful connections between leadership planning, thinking, and actions that systematically improved inclusion in districts and schools. Several rounds of reviewing the interview transcripts and artifacts informed new codes to be added to the growing list of themes emerging from the excerpts, which were then reviewed and compared to the other codes (Loreman, 2007, for example), allowing the patterns to be analyzed (Saldaña, 2021). This step supported addressing research question 2, What is the relationship between Loreman's (2007) Pillars and 
components (systems, processes, and practices) that leaders described as contributing to students with ID being educated in the general education classroom?

A final round of analysis supported an answer for research question 3: How do leaders across different district contexts describe (1) their own attitudes and (2) educator attitudes about the inclusion of students with ID and the district's efforts to address those attitudes? This analysis consisted of separating component data sets and analyzing code application frequency counts within each variable on addressing attitudinal barriers (Avramidis \& Norwich, 2002).

Operational definitions used in analysis:

- Components of Inclusive Education are constituent parts of an educational agency that contribute toward improvement in inclusive education for students with ID, including systems, processes, or practices at all levels of the educational organization.

- Systems: A series of steps that will accomplish a specific goal (district-level policy/priority).

- Processes: A set of procedures to accomplish certain objectives within a given context (building-level policy/priority).

- Practices: Activities that teachers and staff do with students that are predicted to achieve an outcome (teacher-student level).

\section{Presentation and Interpretation of Findings}

This section connects the findings of this study to address each research question. As the data were collected and coded through multiple rounds, themes emerged about how leaders thought, planned, and acted toward improvement of inclusive practices in their districts and schools. The results of this study are based on 
what leaders in three districts describe as the most important aspects of improving inclusive education. Many components (systems, processes, and practices) were described by leaders and, through the analysis, those components informed and reinforced major themes due to the frequency with which they were described and the importance leaders ascribed to them. This study found that aligned leadership, establishing a culture of inclusion, and structures of support (grouping several highfrequency components) all significantly contributed to the improvement of inclusive education for students with ID in the participating districts. Additionally, this study examined the role of educator attitude and ableism as a barrier to improving inclusive practice and found that leaders in these districts and schools addressed ableism and negative attitudes towards inclusion indirectly through structural changes.

\section{Research Question One}

This study's first research question asked: what are the commonalities and differences in components (systems, processes, and practices) that administrators identify to promote the inclusion of students with ID in the general education classroom? Table 2 illustrates the themes that appeared most frequently in the data collection, referred to as components of inclusive education, as described by leaders. Table 2 is organized in descending order by frequency count, with the most frequently occurring components at the top. Three major findings answer the question. 


\section{Table 2}

\section{Component Description and Code Count Component}

\section{Description}

District leaders describe the importance of leaders having an understanding of the importance of inclusive practices, shared vision, and leadership practices that work together toward inclusive practices as a priority from the building level through every aspect of the

Aligned leadership horizontally and vertically

\begin{tabular}{|c|c|c|}
\hline Aligned leadership horizontally and vertically & $\begin{array}{l}\text { District leaders describe the importance of } \\
\text { leaders having an understanding of the } \\
\text { importance of inclusive practices, shared vision, } \\
\text { and leadership practices that work together } \\
\text { toward inclusive practices as a priority from the } \\
\text { building level through every aspect of the } \\
\text { organization }\end{array}$ & 78 \\
\hline Principal leadership is supportive & $\begin{array}{l}\text { An acknowledgment that principal leadership is } \\
\text { critical to the success of inclusive practice. Efforts } \\
\text { or priorities of principal support is included in this } \\
\text { code }\end{array}$ & 59 \\
\hline Creating inclusive culture & $\begin{array}{l}\text { Deliberate efforts to normalize a culture } \\
\text { reinforcing the belief that all students belong in } \\
\text { regular classrooms }\end{array}$ & 56 \\
\hline Training for inclusive practices & $\begin{array}{l}\text { Refers to deliberate efforts at the district and } \\
\text { school level to increase staff capacity for } \\
\text { implementing inclusive practices }\end{array}$ & 54 \\
\hline Strategic teaming & $\begin{array}{l}\text { Deliberate teaming structures put in place in } \\
\text { order to support inclusive practices and problem- } \\
\text { solve challenges that come up }\end{array}$ & 39 \\
\hline Reshaping roles & $\begin{array}{l}\text { Refers to how educational leaders support } \\
\text { educators to think differently about their training, } \\
\text { roles, and responsibilities }\end{array}$ & 35 \\
\hline Explicit about equitable outcomes & $\begin{array}{l}\text { Explicitly referencing their goal of equitable } \\
\text { outcomes for all students }\end{array}$ & 29 \\
\hline Equity lens & Using an equity lens prior to decisions being made & 23 \\
\hline Fiscal & $\begin{array}{l}\text { The fiscal supports necessary to improve inclusive } \\
\text { instructional practices are overtly supported by } \\
\text { district and building budgets as a priority and } \\
\text { approved by the district budget committee }\end{array}$ & 23 \\
\hline Mission/Vision & $\begin{array}{l}\text { Related to a clear mission and vision, with a } \\
\text { shared understanding as to the 'why' of inclusive } \\
\text { practices }\end{array}$ & 23 \\
\hline Empowering teachers and teacher leaders & $\begin{array}{l}\text { Efforts made to give teachers autonomy, support, } \\
\text { and guidance so they own the growth of inclusive } \\
\text { practices }\end{array}$ & 22 \\
\hline
\end{tabular}

\section{Count}




\begin{tabular}{|c|c|c|}
\hline Narrative & $\begin{array}{l}\text { Being intentional about sharing the stories of } \\
\text { success far and wide, in multiple ways }\end{array}$ & 21 \\
\hline Improvement process with small teams & PLCs, Data Teams, PDSA Cycles & 20 \\
\hline High quality instruction & $\begin{array}{l}\text { Refers to efforts to improve high quality } \\
\text { instruction that reaches a wider variety of } \\
\text { learners }\end{array}$ & 19 \\
\hline UDL practices & $\begin{array}{l}\text { Deliberate efforts by a school or district to } \\
\text { increase knowledge and practices with the } \\
\text { Universal Design for Learning framework }\end{array}$ & 19 \\
\hline Aligning inclusion with grade level standards & $\begin{array}{l}\text { Efforts to write standards-based IEPs and align } \\
\text { special education services to support grade level } \\
\text { standards }\end{array}$ & 17 \\
\hline Case-management assignment & $\begin{array}{l}\text { Tactical effort by a district or school to assign } \\
\text { case-management in a strategic way to achieve } \\
\text { improvements in inclusive practices }\end{array}$ & 13 \\
\hline Prioritizing scheduling planning time & $\begin{array}{l}\text { Refers to building master schedules for schools } \\
\text { with additional planning time built in and } \\
\text { deliberate teaming structures at multiple levels of } \\
\text { the organization with the intent of improving } \\
\text { inclusive practices }\end{array}$ & 13 \\
\hline Addressing behavioral supports, specifically & $\begin{array}{l}\text { Efforts made to build capacity to support student } \\
\text { behavior across the general education system }\end{array}$ & 12 \\
\hline $\begin{array}{l}\text { Inclusion for students with ID is a strategic } \\
\text { priority }\end{array}$ & $\begin{array}{l}\text { Inclusion for students with intellectual disabilities } \\
\text { is a school board strategic priority }\end{array}$ & 12 \\
\hline Guidance for inclusive practices & $\begin{array}{l}\text { District publishes guidance for staff with the goal } \\
\text { of increasing inclusive practices }\end{array}$ & 11 \\
\hline Physical space & $\begin{array}{l}\text { The physical arrangement of the educational } \\
\text { environment is attended to for accessibility }\end{array}$ & 11 \\
\hline Hiring practices & $\begin{array}{l}\text { Administration has established intention around } \\
\text { hiring quality teachers and staff who fit with the } \\
\text { goals of increasing inclusive practice }\end{array}$ & 10 \\
\hline Policy & $\begin{array}{l}\text { District policy (e.g., board policy, standard } \\
\text { operating procedures) affirms the district's } \\
\text { intended movement towards increased inclusive } \\
\text { practice }\end{array}$ & 10 \\
\hline Co-planning & $\begin{array}{l}\text { Co-planning is set up through scheduling so } \\
\text { teachers and specialists can plan together for } \\
\text { improved instructional access for all learners to } \\
\text { high quality instruction }\end{array}$ & 9 \\
\hline
\end{tabular}




\begin{tabular}{|c|c|c|}
\hline $\begin{array}{l}\text { Family/parent voice/meaningful } \\
\text { participation/partnership }\end{array}$ & $\begin{array}{l}\text { Intentional effort to seek parent or family voice to } \\
\text { improve participation in the process }\end{array}$ & 9 \\
\hline Assessment/Indicators of inclusive practice & $\begin{array}{l}\text { District tools to assess and progress monitor } \\
\text { indicators that predict improvement in inclusive } \\
\text { practices }\end{array}$ & 8 \\
\hline Co-teaching & The district has implemented & 8 \\
\hline Modeling inclusive practices/systems & $\begin{array}{l}\text { The opportunity for stakeholders to observe } \\
\text { inclusive practices in the classroom, schools and } \\
\text { districts (e.g., through learning walks) }\end{array}$ & 7 \\
\hline $\begin{array}{l}\text { Access to accommodations in the classroom } \\
\text { for all students }\end{array}$ & $\begin{array}{l}\text { A deliberate effort to improve access for students } \\
\text { with disabilities using accommodations }\end{array}$ & 6 \\
\hline IEP process & $\begin{array}{l}\text { Addressing the IEP process in a way that moves } \\
\text { the student and the team toward inclusive } \\
\text { practices }\end{array}$ & 5 \\
\hline Teacher evaluation & $\begin{array}{l}\text { Connecting teacher evaluation with frameworks } \\
\text { for high quality teaching and learning and } \\
\text { inclusive practices }\end{array}$ & 5 \\
\hline Self-determination and student voice & $\begin{array}{l}\text { Deliberate efforts at including students in their } \\
\text { IEP process, especially during placement } \\
\text { determinations }\end{array}$ & $\mid 4$ \\
\hline Access to AT for all students in the classroom & $\begin{array}{l}\text { A deliberate effort to improve practices with } \\
\text { assistive technology in order for students with } \\
\text { disabilities to better access the general education } \\
\text { curriculum }\end{array}$ & 2 \\
\hline Peer supports & $\begin{array}{l}\text { Intentional practice of employing natural peers } \\
\text { (other students) as supports for students with } \\
\text { disabilities to increase access }\end{array}$ & 2 \\
\hline
\end{tabular}

Finding One, Aligned Leadership. Leaders horizontally and vertically in the organization share similar thinking, planning, and actions toward the same goal of improving inclusive practices. Aligned leadership in a school district is essential to setting the stage for improving inclusive practices at the school level. In other words, in these school systems, all leaders were fully committed to and supportive of the district's 
focus on improving inclusive education. Across all levels of leadership-from the school board and superintendent, to executive leadership, in all departments, and for every principal-leaders understand why moving to inclusive practices is important and understand their role in that work. District leaders describe resistance from reluctant staff and community members as one challenge of moving toward inclusive practices in the classroom. For these leaders, in their districts, aligned leadership created a united community of practice to gird against resistance to the notion of having students with disabilities learning with their peers in the general education setting. One district leader described the importance:

I don't know how you would ever do this work if the superintendent wasn't passionate about it . . . and the principals, because the community is going to have certain people that are going to push back. They are going to look for a ... weak link. You can't let them find it or have that weak link be there.

As this district leader describes the dynamic, the importance of executive leadership support is highlighted as essential and necessary as the chief executive officer (superintendent) answers to the board, representative of the community.

Principal Leadership. Inclusive practices in a school would not take place without strong principal leadership leading the improvement effort. Each participating district, at each level of leadership, described and affirmed principal leadership as one of the most important components of schools moving toward inclusive education. In order to improve and move toward inclusive practices, principals need autonomy, trust, support, expertise, and resources. A principal described this need: 
I got to decide how is that team going to work together, how are we going to figure out who is going to support who, how/what's my role, and deciding when the kids in the classroom . . . might need some additional support . . . I had a lot of oversight and say and how [inclusion] actualized.

Principals can leverage both their relationships with building staff and their role as the primary instructional leader to effect improved inclusive practices. They are able to manage problems as they arise and shift resources locally in order to support teachers in classrooms. One principal said, “. . . building leadership had to really help shape everyone's thinking ... how to bring everybody on board ... help everybody understand why we wanted to move to inclusion, why it was so imperative to do so." The principal must believe in the value of the work as they lead the day-to-day problemsolving and coordination of support efforts to improve inclusive practices. While every school in Oregon is unique and has its own culture, a school principal does not operate in isolation. One principal offered:

I could not have done this as a building leader if I didn't know that [the special education director] had my back no matter what . . . I think it starts top down, down, up, as an organization, because if there was [sic] multiple points along the way where if I didn't have the district level support and backing I would have stopped.

The principal's leadership operates within the larger context of their district. Each school is accountable to its community, by way of an elected board of directors. If a parent in the community has questions or concerns about students with disabilities in the regular classroom setting, and is not satisfied from working with the principal, they can work with district leadership or the school board, which underscores the importance of aligned leadership. 
Equitable Outcomes as the Goal. Leaders across the districts described intentionality behind messaging the goal of moving toward inclusive practice as working toward equitable outcomes. Equitable outcomes refers to explicitly applying a school's goals (achievement, for example) to all learners. To work towards equitable outcomes requires resources within the school being leveraged toward bringing all learners along toward high achievement, recognizing that some learners will need more support than others. One district leader described the importance of this conceptual frame and language:

So what happens is that we become familiar with it [the conceptual frame of equitable outcomes], teachers also become familiar with it, so you get this shared language where people are talking about the same things, and start to value the same things. It becomes pervasive so that it weaves its way into other layers of planning ... to the point where it almost becomes unconscious. Aligned leadership horizontally and vertically in the system is a key strategy participants used to ensure that the districts shared common goals of equitable outcomes and increased inclusive practice. Principals are a key determinant in establishing these goals at a building level, but their work must be supported by leadership throughout the system.

Working towards equitable outcomes was common amongst leaders interviewed for this study and was equally evidenced in a review of district artifacts. Equitable outcomes emerged in policy, procedures, strategic planning, and within the culture of leadership.

Finding Two, Culture of Inclusion. This section will break down the component strategies leaders used to create a common culture of inclusion. Participating districts had intentionally established cultures of inclusion. Each district shared artifacts (e.g., policies, procedures, equity action plans, district strategic priorities across all bounded systems described) that articulated and specifically directed efforts to create a culture of 
belonging for each student. Leaders described the transformative impact that this intentionality can have. For example, explicitly teaching lessons to students about person-first language for people experiencing differences or disabilities can shift attitudes and narratives about disability. A teaching and learning director described how narrative influences culture:

The culture of most organizations is to talk about the negative-not that people want to focus on it, but because it is the immediate problem they want to solve. And we'll never run out of those problems! So we have to make space for where [we] are successful. Most great teachers will have a thousand successes and they'll go home, obsessing about the one non-success of the day. So our role as leaders is to create a culture where we also talk about success, you know, we're not trivializing it, these are real stories that show people are making a difference.

This leader articulates the importance of sharing success stories as important to shape the conversation, which then shifts the culture. A special education director described this dynamic as supportive of the work because every leader faces periods of doubt, but an intentionally inclusive culture gives permission to unapologetically support one another in the work. This leader says, "we may not want to say it but we waver ... because of the myriad of situations ... we have to be a whole system and [have] a structure that can support" one another through challenges.

Expert Learners. Educator beliefs in support of inclusive teaching can support the culture of the school community. One such belief is the notion that creating expert learners is a pedagogical goal that serves all learners. When educating a diverse group of learners, this aspect of teaching and learning is an important ingredient. The ability to help students become expert learners is essential to inclusive education. Leaders 
described this connection between a culture of inclusion and creating expert learners.

As one teaching and learning director states:

... we've recognized the value of heterogeneous classrooms, and we were a district that, for many years, had blends - had blended grades at every school. And so it goes back to this idea that when you have a classroom with a great deal of diversity, and everyone is learning a great deal of skills to be successful. That connects to the idea that you can expect that they are going to rise up - that a 3 rd grader is going to be successful in a classroom with 4 th graders.

A culture of inclusion, in turn, reinforces educator belief that all students can become expert learners. Having an inclusive culture thus helps shift educator practices to being more inclusive in nature.

Ownership. In light of historical context and teachers' tendency to take responsibility for student learning, ownership of student learning was a nuanced aspect of school culture that leaders need to address in order to improve inclusive practices. For example, while case-management for students with disabilities is a necessary service delivery approach, especially given the significant procedural requirements of the IDEA, the practice may inhibit inclusive practice because the special education teacher responsible for case-management often takes ownership of that student's learning. Leaders interviewed for this study described that this ownership went beyond ensuring that the services indicated on a student's IEP were provided. Rather, from the perspective of leaders who participated in this study, specialists have a tendency to take ownership of student learning outside of the scope of their practice (e.g., core instruction in general education) due to fear that the student would fail. Leaders described reluctance on the part of the specialist, in some cases, to let the general 
education teacher(s) have a significant role in a student's education. One principal described structural adjustments at the school-changing specialized classrooms to general program classes - as a way to shift the thinking about who should take responsibility for students with disabilities' learning. The principal said:

... it took multiple messages and ... meeting[s] ... [sharing that] these [students with ID] are our students, we are going to serve and ... [they should] have those same opportunities [as all students]. So, it became, you know, shifting that mindset from "their" students. You know the "Oh those are [the special education teacher's] kids" to "those are our kids."

Principals interviewed for this study described both structural changes (e.g., dismantling specialized programs) and addressing ownership when it is a barrier with staff in order to help the thinking evolve toward inclusive practices. One principal described:

I'm working right now with a learning specialist who is struggling with . . . this need to have that ownership and control. What's happening is that the students that they're supporting are not having the same inclusion experience that I would want them to have ... Their attitude ... is getting in the way ... ultimately we do need to help our learning specialist see that they are providing a service, and in pursuit of having all children have the access.

Many schools that are moving toward inclusive practices have effectively dismantled segregated programs set in special classes (e.g., life skills classrooms, communication classrooms, behavior programs). Staff teaching in segregated settings appropriately take significant ownership of their students' educational experiences. As programs shift towards inclusion, so does the responsibility of ownership over students' educational experiences. The principals interviewed for this study described the adaptive shifts necessary to support a change in role for their staffs from solely owned by a specialist to collaboratively owned by a team, with general education primarily responsible. 
Finding Three, Structures of Support. Leaders in this study described efforts to improve inclusive practices as more successful when appropriate structures of support have been established in their schools. While structures of support refer to varied components identified by districts as essential, this study highlights the three most important support structures in the minds of those leaders interviewed: training for inclusive practices, teaming structures, and paying attention to role clarification. Participants indicated strong agreement that it is essential that these three structures of support exist across their system, at varying levels in the organizations, in order to improve inclusive practice.

Training. Training educators for inclusive practice demands a clear plan and investment in resources and time. Participant interviews and artifacts suggested that training is a significant component of inclusive education. As one Teaching and Learning Director described, "sometimes teachers feel like they don't yet have the skillset, or the supports, or the professional knowledge, or the class size, or the curriculum. Those are the things they want help with." Interview participants for this study described how leaders thought through and planned to address this need proactively and in an ongoing way. One district leader outlined their approach:

One of the favorite parts about my job is leading a class we do for all of our new teachers .... around the culture of our district. We have four sessions where we meet $\mathrm{K}-12$, led by high status teachers in our district, and our very first session is always on equity and inclusion ... it sets the stage for that inclusion.

Training and professional development must be targeted, sustained, and supported over time. Training must address the skills of all staff in the school, and be 
supported by the larger district initiatives. Leaders interviewed for this study described that inclusive practices are identified in each of their school's continuous improvement planning process, and as part of the school's professional development plan. All staff involved are essential stakeholders in improving inclusive practices. For example, leaders described specialists such as special education teachers and speech-language pathologists were included in the general education professional development when general education staff are working on curriculum mapping for state academic standards. Including specialists allow them to connect student IEP goals to the skills needed to demonstrate mastery of the standards that general education teachers are teaching to all students.

Training Across the System. Leaders described the shared planning involved every year to make sure barriers to inclusion were addressed through professional learning that includes general and special education teachers, paraeducators, bus drivers, custodians, secretarial staff, and administrators. One special education director described efforts to fold specialists into professional development opportunities with general education teachers:

... our special educators are always included in the professional development, as ... simple as even making sure that [they are included in] staff meetings where we're going over the reading curriculum, the math curriculum.

A Teaching and Learning Director added:

... the expertise of your staff is so important. There is an opportunity when a school district makes this shift, because you have some people who are so expert because this is all they have been doing, and so, we need to consider how we celebrate their status in ways that build confidence for the people who may be 
afraid, because they've never had the opportunity to work with some of these kids.

Leaders described targeted training of specialists, such as special education teachers, allow them to build content area expertise to support their provision of specially designed instruction. Leaders described the importance of planning a scope and sequence of training so specialists could effectively support students to understand content being taught in the general education classrooms. Training or professional development for all involved was reported as an essential structure of support to have in place in order to improve and sustain inclusive practices.

Teaming. Teaming, as described by leaders in this study, refers to intentionally scheduling time for varied expertise to collaborate in order to solve the problems and challenges that come with an inclusive educational model. Examples of teaming structures to support inclusive education frequently emerged from the interviews and artifacts including, for example, IEP teams, improvement teams, co-planning, and gradelevel teams. As a middle school principal described:

... you need to have dedicated time to build the capacity of everyone to do this work, because it means collaborating in ways they've not collaborated before. It means planning, the way that they've not planned before ... providing PLC time ... providing time for teachers to come together and share what's working, what's not working.

Team structures need scheduled time, effective processes, and clear objectives, according to leaders.

Leaders noted that, because there is a difference between a group of people and a team, it is important to intentionally and explicitly cultivate the conditions for people 
to work together as a team. Leaders described their efforts to build a team (e.g., scheduling, established norms for working together) as helpful in moving their staff from cooperation to collaboration. Leaders described this collaboration as being generative toward solutions together. Effective collaboration within and across their teaming structures is an essential hallmark of an inclusive culture. A principal emphasized this succinctly when they noted, "we have to have . . . a culture of collaboration to support students ..." While the concept of teaming and collaboration is understood by educators, leaders described that it does take attention and facilitation to ensure that professionals are collaboratively working together as teams, as opposed to cooperating or completing tasks as a group.

Teaming processes benefit from intentionally structured supports in place like following a prescribed set of procedures. For instance, improvement science helps teams identify barriers, ideas for solutions, and ways to measure improvement to confirm whether a practice or strategy is working in order to determine whether to continue or shift to another idea. District 1 leaders described a firm commitment at all levels of the organization to the process of improvement science. A key facet of improvement science is that all staff are given permission to abandon practices that are not effective, which supports continued progress toward their goals. Each leader described that they have their own Leader Action Plan with established goals. They are accountable to their own goals on a regular basis. The Teaching and Learning Director described, "our buildings all own what we're doing in improvement science. They have 
set goals, they have leader action plans, and ... our PLC teams have developed leader action plans to continue that cycle of inquiry."

There were other components leaders described as important, even essential, but there was not the level of frequency of mentions and discussions as there were in the major findings. Therefore, some of these important components were not discussed in the results section. A few examples of important components include: making decisions using an equity lens, fiscal supports, the importance of mission and vision for a district, empowering teacher leadership, and high-quality instructional practices such as $U D L$, to name a few. Another nuance that was mentioned in the analysis was the importance of aligning IEP goals with grade-level standards. Writing standards-based IEP goals was described as an important aspect to improving inclusive practices as it effectively aligns special education services with the work the general education is doing in the classroom. Other examples of components that are worth mentioning are physical space and physical access to students with disabilities to be fully included in the general education environment.

The pace of change was another nuanced theme in the data that did not land in a component or major finding, but an important dynamic at play when thinking about systems change. This dynamic of the pace of change suggests being mindful of which steps to take and when. There are indicators as suggested by the leaders in this study. Taking stock and regularly assessing progress to assure meaningful inclusive practice is taking place is important as once a goal is reached, looking toward and planning the next step is essential. Regularly surveying teachers, support staff and building leaders is 
important to keep a sense of those markers of readiness. Reviewing student progress toward IEP goals for students experiencing ID and learning in the general education setting is important to monitoring effective and meaningful practice.

\section{Research Question Two}

This study's second research question asked: what is the relationship between Loreman's (2007) Pillars and Components (systems, processes, and practices) that leaders describe contribute to students with ID being educated in the general education classroom? After the first round of coding, a second round of coding organized the components (see Table 2) within Loreman's Pillars (see Figures 8 and 9). Figure 8 provides a graphic representation of the frequency of components in terms of code counts when organized by each pillar. In other words, Figure 8 illustrates how many times a leader described or referenced one of Loreman's Pillars as an aspect of improving inclusive education. Figure 9 shows the co-occurrence of codes of the most frequently identified Components and Loreman's Pillars. The Pillars that emerged in this analysis are Policy and Leadership (P2), School \& Classroom Processes (P3), and Training \& Resources (P7). Organization of the codes in this way is helpful in shaping the discussion of findings below. 


\section{Figure 8}

Total Code Co-occurrence of Components of Loreman's (2007) Pillars

Frequency of Components within Loreman's (2007) Pillars 1000

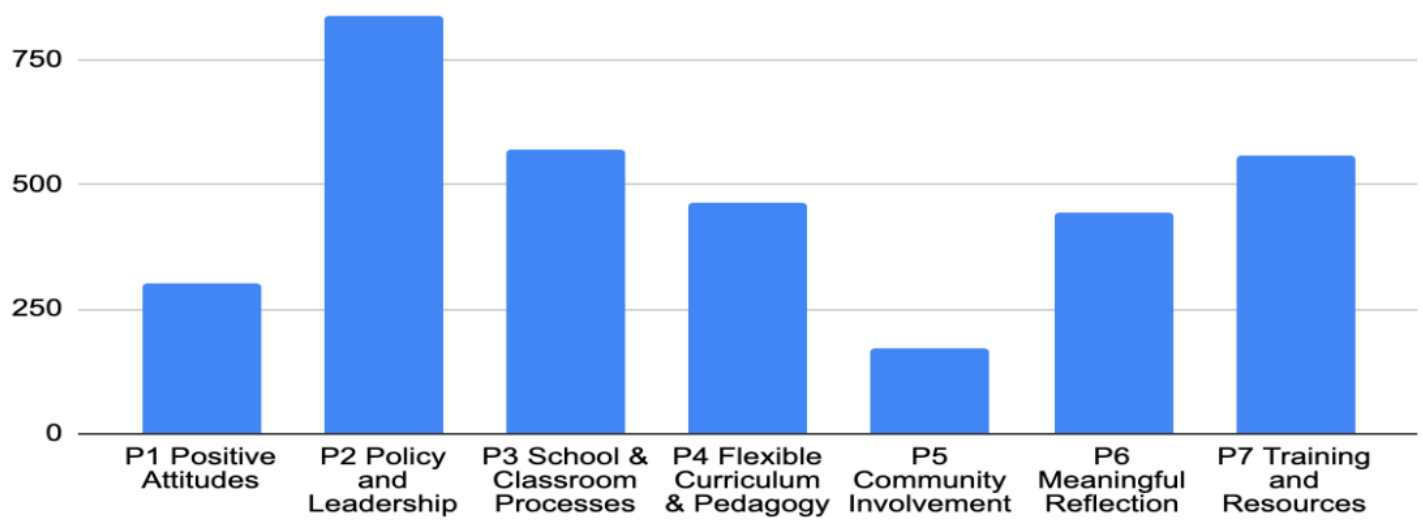

\section{Figure 9}

\section{Code Co-Occurrence of Components and Pillars}

High Frequency Components of Inclusive Education and Loreman's (2007) Pillars (code co-occurrence frequency)

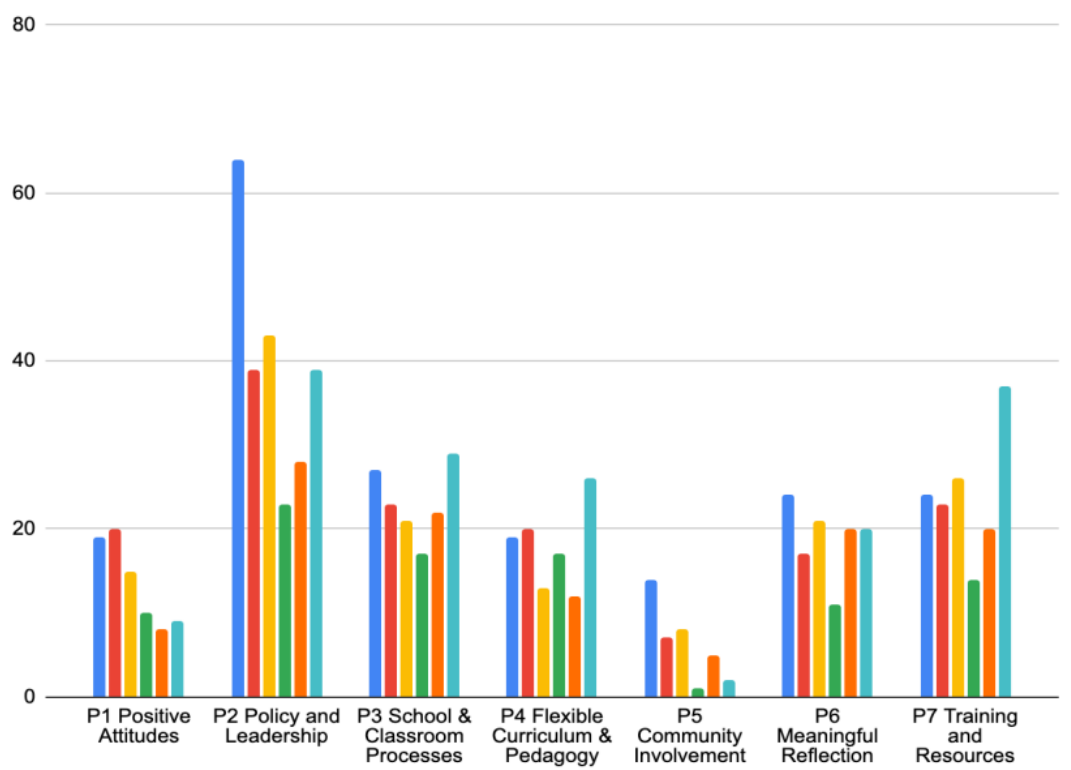


Aligned leadership. Aligned leadership was strongly connected with Loreman's (2007) Pillar 2: Policy \& Leadership. Leaders in this study emphasized the importance of policy in support of improving inclusive education (identified priorities) and that all leaders needed to believe in the work. In order to improve and increase inclusion in their district, leaders described how they needed to feel the support of one another through a common and shared vision of the value inclusive education brings to their schools and communities.

Finding one in this study highlights that the most important aspect of improving inclusive education is for districts to assure that leadership (horizontally and vertically) shares the same vision for inclusive education, values the work, and is ready to defend it. Loreman's (2007) Pillar of Policy and Leadership highlights the need for supportive policy at multiple levels of governance and in building level leadership; this study confirms that all leaders in the system must be aligned in order for inclusive education to improve at the school level. A teaching and learning director highlighted the importance of leadership in moving systems towards inclusion because there will be resistance. This director described the need for aligned and united leadership:

I don't know how you would ever do this work, if the superintendent wasn't passionate about it, the assistant superintendents weren't passionate about it, and the principals, because the community is going to have certain people that are going to push back. They are going to look for a chink in the armor, or a weak link. You can't let them find it or have that that weak link be there. And so I think leadership, you know, leadership matters.

Findings from this study indicate that leadership is essential in improving inclusive education. Principals align leadership within their building (horizontal alignment) 
towards inclusion, but the leadership context that supports that school must be aligned and on board throughout the entire organization (vertical alignment).

Culture. The importance of creating a culture of inclusion has high code cooccurrence in each of Loreman's (2007) Pillars (see Figure 9). In other words, creating a culture of inclusion contributes significantly to all seven pillars and indicates a high degree of overlap. As illustrated in Figure 9, creating an inclusive culture is one of the highest frequency counts of the component counts in each of the pillars. In particular, a culture of inclusion supports growth in Pillar 1, Positive Attitudes. The finding in this study on the importance of an inclusive culture affirms the impact of culture on supporting attitudinal change. One teaching and learning director said:

years we've recognized the value of heterogenous classrooms, and we were a district that, for many years, had blends - had blended grades at every school. And so it goes back to this idea that when you have a classroom with a great deal of diversity, and everyone is learning a great deal of skills to be successful. That connects to the idea that you have to show students that you believe in them and that you can expect that they are going to rise up-that a 3rd grader is going to be successful in a classroom with 4th graders.

This district leader described the levels of diversity in classrooms as normalizing the practice of teaching to a wide range of students.

Structures of Support. The finding that structures of support in a school are an essential component of inclusive education is connected to the theoretical frameworks of this study through each of Loreman's (2007) Pillars. However, the elements of training or professional development and strategic teaming was described the most by leaders (see Figure 9). Participant interviews and district artifacts referenced training 
and professional development for inclusive practices 54 times (see Table 2). As such, training for inclusive practices was the highest co-occurring component in Pillar 7 (training), Pillar 4 (flexible curriculum \& pedagogy), and Pillar 3 (school \& classroom processes). While Loreman (2007) described training in Pillar 7, he approached it from the lens of university preparing staff outside of the school system. This study revealed a substantially different approach. Leaders interviewed for this study conceived of the necessary training and professional development as an in-house responsibility, to be led by district, school, and teacher leaders. They described how essential it is for professional development priorities to support the work of improving outcomes for all learners, like training on and unpacking learning standards for all teachers and specialists. One principal described supporting teachers and specialists to understand how critical it is if you want a sixth grader to be able to interact with sixth grade standards, they have to be in a classroom with the teacher who deeply understands those standards ...

As part of the structures of support, strategic teaming co-occurred at a high level in many of the pillars (see Figure 9). The ability to support school teams to plan together, learn together and problem-solve together is essential and connected to Pillar 2 (classroom processes), Pillar 6 (meaningful reflection), and Pillar 7 (training and resources). One principal described that

once they started having this great connection and it started working. And then, of course, the buzz around the building, you know, spread and so then more and more teachers embraced it, and so now it is just the way we do things. So when the two math teachers for seventh grade meet, they also meet with the learning specialist, and they all plan together so that the co-planning is happening and then the co-teaching occurs as well. 
Organizational Efficiency to Address Structures of Support. Loreman (2007)

described the need for addressing organizational factors that impact the school's ability to successfully provide inclusive settings, such as the school schedule. Participants in this study revealed an interesting theme related to this need, with a surprising facet: when addressing organizational factors, Oregon administrators must find efficiencies in order to sustain this work, especially in light of inadequate resources and insufficient support to meet the myriad requirements for public education. Oregon schools have consistently had to function with restricted resources. Given the funding structure of Oregon schools as outlined by the Quality Education Commission (2020), even after the historic passage of the Student Success Act (Oregon Department of Education, 2020), Oregon administrators must lead systems in the face of being underfunded and underresourced. As one special education director describes:

I do think that the way that Oregon funds their schools . . . we can't do that [reasonable caseloads] because we don't have the FTE [the number of Full-Time Equivalent staff employed] that is typically allocated and some of these East Coast schools ... we don't even have close to that FTE, but they have a case manager for first and second grade, they have a case manager for third and fourth grade another one for fifth and sixth grade and I'm like, "Oh, that's how they do it; they have money." Okay, so, so that means that we need to take the resources that we have, we need to build a model that is sustainable.

School leaders shared that, given available resources, they must operate efficiently in order to attend to the structures necessary to improve inclusive schooling, like planning, scheduling, monitoring, and evaluation.

District 1 leaders shared a notable organizational structure in their practice that supported increased inclusion. Their leaders described a supported accountability 
practice that empowers each leader in each department through a leader action plan. Under this mechanism, each leader develops their own plan that describes how they are working toward the organization's priorities, with accountability steps every 45 days.

This structure allows leaders to manage their current situation while keeping a significant focus on improvements that contribute to the organization's strategic priorities. For District 1, one of their priorities is improving inclusive practices, which lends significant organizational support to this work. One principal described this organizational support:

[The leader action plan] keeps us focused on the right things. We're not ... [pulled] to the next big thing ... we're held accountable ... it streamlines our work and, if [a demand] doesn't fall into that [plan], you can let it go. Because principals-we know we have so many balls in the air, at some point you are going to let things go. And so it's okay to do that if it's something that doesn't align to what we're working on, and kind of almost gives ... permission to prioritize what we said was important ... I know exactly what I need to do [and] my expectations are clear.

Attending to structural changes is essential to improving inclusive practices, but participants in this study revealed that appropriate organizational support can keep these changes from being overwhelming. Leaders also shared that a key to managing this change alongside the many other demands placed on educational leaders today is recognizing that improvement in inclusive practices is also a solution to many of the equity problems schools face today. In this regard, a special education director noted, "we're working on it [inclusion] as part of our equity work and we're really focusing on it again and so I'm super optimistic that ... the data [for inclusive placements] is ... going to look better and better." Leaders in this study see inclusive practice as excellent 
instruction in the general education classroom that reaches a diverse group of learners. Accordingly, findings from this study support the concept that inclusive practices benefit students with disabilities and address opportunity gaps for all groups of students.

Reshaping Educator Roles. Leaders described reshaping staff roles as essential to improving inclusive education at the school for students with ID. Though this finding was not directly represented in the theoretical frameworks used, the notion of shaping educator roles does overlap with Pillar 1 (positive attitudes), Pillar 6 (meaningful reflection), and Pillar 7 (training and resources). The strongest code co-occurrence of this component in this study was in Pillar 2 (policy \& leadership). One principal described a communication to a special education teacher reluctant to see their role differently:

I know you ... got into this work because you really wanted to ... be a teacher, and you wanted to see the progress that they're making and really . . . be there on the front line when it happens. But, ultimately, if we want our students to have access to the math teacher, to the science teacher, we have to find ways to have the math and science teacher be their teacher, with you supporting them to access the content.

This principal described how important it is to reshape the work of their special education specialists to see that they need to support access for students with disabilities, while not denying general education ownership over student learning for students with disabilities.

Leaders interviewed in this study described a dynamic tension that exists when working to shift educator roles. Not all educators are able or willing to make the shift. One principal described such a situation: 
When we went to this model, there was going to be some significant change for some of our teachers, for some of our students, as you know, all of a sudden they're not being served in a life skills classroom ... we're not taking the life skills program away. They're just not going to have a classroom where they sit and play Uno every day for a period with an assistant ... we had to communicate that ... I lost a case manager in the mix ... [a] really great life skills ... case manager that said, "this isn't me," I want to be a life skills teacher. And I want that program class. And so she took a transfer to another building.

The importance of role clarification that leaders described was surprising because it was not explicitly named in Loreman's (2007) Pillars.

\section{Research Question Three}

This study's third research question asked: how do leaders across different district contexts describe (a) their own attitudes and (b) educator attitudes about the inclusion of students with ID and the district's efforts to address those attitudes? All of the major findings in this study contribute to answering this question. Aligned leadership, an inclusive culture, and structures of support all create a deliberate focus on equity by moving toward inclusion. Those structures also point to the fourth major finding about addressing attitudes influenced by ableist thinking in order to reduce segregated settings. The fourth major finding in this study answers research question three with an understanding of how leaders described addressing attitudinal barriers, indirectly, through organizational structures of support.

Ableism. A surprising finding was that leaders in all three districts described not addressing ableism directly. Rather, leaders shared that they generally addressed ableism and deficit thinking indirectly through structural changes; only in cases where ableism caused a significant performance problem was it addressed directly. By 
reviewing the artifacts collected related to how districts address educator attitude (e.g., hiring practices, resource allocation, support for classroom teachers, extensive training for general education teachers and specialists), it is apparent that-while countering ableism is not explicitly planned-these leaders make significant efforts to address ableism. One principal interviewed described ableism as:

a huge hurdle. And, and to be quite honest with you, I'm working right now with a learning specialist who is struggling with that, and it's impacting ... you can see that because they have this need to, to have that ownership and control. What's happening is that the students that they're supporting are not having the same inclusion experience that I would want them to have.

Leaders in this study generally described the structural changes for addressing ableism and attitude as creating a culture of inclusion and belonging. One special education director described these efforts:

We haven't explicitly talked ... with staff . . . about ... ableism ... in that way [directly]. I think it's been more of leading by sharing and re-sharing our commitment to our mission, vision, and values. And then supporting people that you know may be struggling, like, okay, we're going to support you in . . y your challenges with this ... concept. And, at the same time, like, I don't want to sound too harsh but you know if this isn't the right district for you, that's okay because here's what we're committed to doing. And, so, you can decide at the end of the school year if this is what you want to continue to do. But it has been difficult, especially the first couple of years.

Leaders discussed attitudinal barriers that were fear-based, and described how they addressed them through a combination of exposing the teacher to new experiences (e.g., classroom observation) and ensuring professional learning to build the requisite skills. One special education director described a survey response from a teacher, "I don't know what to do. I've never had a student with down syndrome ... I 
have no idea what to do with them." Leaders from this study worked to resolve those fears. A principal described coaching resistant teachers by building on something the teacher is already confident that they can do:

[the fear is] ... we don't have the training for this [inclusive classrooms] . . . let's take it back a step .... If we really believe ... in building relationships with our kids, you have the capacity [and] we're able to do this work ... I had to really be crystal clear, almost to the point where I was saying, don't teach academics ... those first few weeks . . . because I'm telling you that relationship ... is the most important key to ever getting to academics.

In this example, the principal described directly addressing the fear by using the teacher's strength of building relationships. The principal described scaffolding this core skill the teacher possessed by coaching and giving permission to focus on relationships before academic instruction. As a result, the principal shared, the teacher developed that skill and their attitude shifted to being more open and more confident with a wider range of learners in their classroom.

Another nuanced structural change leader shared was paying attention to small details that impact teachers, such as identifying the teacher of record for a student. One teaching and learning director described:

... we used to locate our program classes [segregated settings with students experiencing disability] in a home school ... In our SIS [student information system], those students were rostered under the classroom of the special education teacher. At some point, working in the year, we decided we would roster these kids in their home classroom. Their teacher will know ahead of time and they can prepare for the student like a cubby, a name on the desk, their folders, emailing parents. That subtle shift set not only set the stage for those students to be seen much more typically, but the teachers said, "Thank you! Every year, you tell me my class size and it's not accurate. Because I am working really hard for this one student, and I don't feel like it is being recognized." And, 
so, by putting it up front, instead of worrying, the teacher would say, "Oh great, I have one more student." A teacher actually said, "Thank you for recognizing how hard I'm working to take care of this child."

In this example, we see recognition as reinforcing for a teacher who is working hard toward inclusion in her classroom. Both the structural shift and the timing of that shift impacted this teacher's attitude.

Leaders describe a dynamic tension between readiness for change and making firm decisions to move students with disabilities into general education classrooms with the support they need to learn alongside their peers. One teaching and learning director explained:

We never would have said, "Okay, we are ready, because we now have all the pieces in place." But we got to the point where we had enough in place, and we knew that we just needed to start trying it to see what we needed to learn. Years and years of principal meetings, visuals to help concepts, professional learning, etc. ... at one point we just decided we weren't going to do [program classes] any more.

A special education director further explained:

You can't ease into it [inclusion]. If you [are] just like, "well we're going to pilot it here and just see how it goes." Or we're going to try it out in this building or this classroom ... you gotta just, you got to rip the band aid off ... we're going to do it and it's going to be ... messy as heck.

These leaders described a similar approach to navigating this tension: prepare and build capacity, but make the change.

Ableism and Racism. While ableism is used in this study as a theoretical framework to view attitudinal barriers with educators showing up as resistant to change, given the connections between ableism and racism, this study also explored the 
intersectionality of race and culture related to segregation and inclusive education. Each participant who was interviewed was asked: How did race and/or culture factor into your equity priorities for improving inclusion in the general education setting for students with ID?

Respondents were mixed in their views. Some participants said the intersectionality of racism and ableism did not factor into their thinking. Other participants reported a deep connection between inclusive practices and culturally relevant teaching. One special education director elaborated:

I think the role of race and ... culture are ... a huge factor in thinking about inclusive practices and how we maneuver this work, in part because we have a history of students ... across our country ... who have been segregated in multiple different ways based on the color of their skin, and over identified in specific . . . disability categories -including ID, is one of those. And then we've seen a trend of then segregation of classroom as well. So, when we think through how do we do our racial equity work, inclusion has to be a foundational rock of it because that's one of the ways that we've seen, historically, students be underserved.

This leader is describing the connection of segregated placements based on disability is an equity matter in their district, and connected to their work toward improving outcomes for all learners. A Teaching and Learning Director discussed the need for intentionality when

looking at race, language, [and] socioeconomic status . . . we are really careful around that language. We use the word cohorts, cohorts of students who may be described by different categories; we are careful and don't want to permanently categorize a student for fear of accidentally attaching other traits that may or may not be true. In looking at data sets, if we have enough of a data set for it to be a valid disaggregation, we do consider that ... we hold that very carefully in our district. We include that in our wonderings: are we reflecting historical disproportionalities that public education has contributed to? And I 
think we've gotten much more comfortable with the idea that, you know, it may not have been our intention, but it's not by accident that public education keeps resulting in the same disproportionalities.

One principal described a specific connection between race and disability that they are working on within their district. The district shared that they have too many modified diplomas assigned, disproportionately, to students of color with disabilities. The principal described a decision point with staff that had become a pattern:

They're really struggling - not sure they're going to make it [to graduation]. Let's talk about a modified diploma and get them across the board. And so, shifting that has been important ... ensuring that we have administrators in the meetings when those [decisions] happen.

Another district described that their demographics were not varied enough to have race and culture a significant driver for decisions; instead, they reported focusing on each student with their individual needs for support in the regular classroom.

Addressing attitudinal barriers for educators when implementing inclusive practices in schools is essential, according to participants in this study. The leaders interviewed reported to have addressed ableism and attitude through establishing and maintaining a culture of inclusion and belonging; providing supports, structures, narrative, and coaching; and maintaining a laser-like focus on equitable outcomes for all students. The intersectionality of race and disability, while tangentially explored in this study, is found to have significant differences between districts that leave more questions than conclusions. This is an excellent area on its own for further exploration with further research. 


\section{Limitations of Study}

District Selection. The school district selection process likely missed schools and districts in the state of Oregon that are demonstrating improved inclusive practices for students with disabilities. The selection process used triangulation by surveying two groups, but yielded a response rate of less than 50\% (3/19 ESD Special Education Directors and 5/10 Oregon Department of Education County Contacts). However, limited responses did not hinder achieving the goal of the study: to understand leadership behaviors across three districts that resulted in improved inclusive practice. Equally, despite the limited response rate, the districts selected to participate provided some level of representativeness of districts in Oregon, as they ranged in size and geographic location. There was also a wide range of grade levels represented, with participant selection resulting in elementary, middle, and high school principals being interviewed.

Participant Focus. The study focused only on selected leader perspectives, as was the aim of this study. While leadership provides an excellent, and essential, source of information, a significant limitation of this study is that it did not add perspectives from those delivering the inclusive practices (i.e., teachers and staff) or those in receipt of inclusive educational services (i.e., students). Only leadership perspectives were gathered.

Virtual Data Collection. Given the restricted nature of completing a study in a pandemic with state restrictions on in-person meetings, all interview data were 
collected virtually through a video conferencing platform. This study is limited in that no site visits were made to observe inclusive leadership in practice. Site visits with field notes would add considerably more depth to the study's findings.

Data Analysis. While an excellent analytic software tool was used, it is only as powerful as the first and second rounds of coding with a novice researcher. Despite multiple rounds of coding, it is possible that certain aspects or nuances were missed as a result of an inexperienced researcher coding datum. Another limitation exists in the charts presented. While it is believed that they generally represent the relationship between variables, they may compel the reader to draw conclusions that go beyond the story they reveal about the relationships between variables (e.g., assumptions of causation between co-occurring codes). 


\section{Chapter 5 Discussion}

The American education system continues to struggle with persistent segregated settings for students with ID (see Figure 3; Brock, 2018; Morningstar et al., 2017). Oregon schools (see Figure 1) struggle with meeting LRE targets (see Table 1) and disproportionately place students who experience ID in more restrictive settings compared to other disability categories. This study outlined the problem of practice and sought to understand how leaders think, plan, and act in order to improve inclusive practices for students with ID. Placement for students with ID is a particularly challenging problem to solve in the context of a state that underfunds its educational system (Quality Education Commission, 2020), operating under rules that compel districts to provide a continuum of alternative placements -including special classes and special schools, or segregated settings. Each eligible student's IEP team must make an individualized determination as to whether a free appropriate public education (FAPE) can be provided in an inclusive setting. The individualized nature of this determination contributes to the challenge of systemically increasing inclusion for students with ID; ableism and deficit views that persist in many IEP team members can result in overly restrictive placements, especially for students with significant disabilities.

Oregon experiences a vast historical context of segregating people with significant disabilities, and only recently has the Oregon education system moved away from preparing students with disabilities for sheltered workshops as specified in the Lane settlement agreement (Lane v. Brown, 2016). Therefore, improving inclusive 
practices, and working toward inclusive education for students with ID is an appropriate area for schools and districts to prioritize. In Oregon, since schools are accountable for post-school outcomes (competitive employment, further training, and college) for students with disabilities, moving away from segregated settings and toward inclusive education is more important than ever. There is a clear and direct link to the benefit of inclusive practices to outcomes for students with ID (Agran et al., 2020; Andreson et al., 2020; Ballard \& Dymond, 2018; Banks \& Polack, 2014; Bouck \& Joshi, 2016; Cosier et al., 2013; Kleinert et al., 2015; Ryndak et al., 2012; Ryndak et al., 2010; Wagner et al., 2006; White \& Weiner, 2004) both during K-12 education, when receiving transition services, and in life after they leave the public education system. When schools strive toward improving clear, unrestricted access to the most appropriate instructional setting for every child, they are setting the foundations for students with disabilities to achieve markedly improved outcomes, and preparing students for increased success when they leave school. If Oregon K-12 schools can raise expectations for students with disabilities and for the staff serving them, students will be better equipped to navigate an adult world not designed to accommodate disabilities.

\section{Synthesis of Findings and Implications}

Leaders in this study described the following components as most essential for promoting or guiding schools toward implementation of inclusive education: aligned leadership, establishing a culture of inclusion, and structures of support (grouping several high-frequency components). Leaders describe each of these elements as critical 
to influencing the technical and adaptive shifts necessary to create the condition in schools where inclusive education can improve for students with ID. Additionally, this study explores the role of educator attitudes and ableism as barriers to improving inclusive practice, and finds that leaders in these districts and schools addressed ableism and negative attitudes towards inclusion indirectly, through structural changes.

\section{Components of Inclusive Education (Research Question One)}

Moving a school community from segregated placements for students with ID to improve inclusive practices is a complex endeavor (SISEP, 2020). Doing so requires the leaders of the system to identify segregated education (with related outcomes) as problematic and worthy of attention and a priority. School leaders then must craft a unified vision with the school community and all stakeholders, especially school and district leaders. It will involve deliberate and intentional efforts to create a narrative and culture that puts value on belonging and the diversity of learners in general education classrooms. It will involve a commitment of financial resources to support the work, and support structures.

This study, focusing on investigating leadership efforts for improving inclusive education, found that there are significant similarities in how districts of different sizes, approached improving inclusive education and implementing inclusive practices. The major findings suggest aligning leaders and connecting district priorities, creating a culture of inclusion and belonging, and implementing strategic support structure are essential to improving inclusion. In essence, leaders established a policy and expectation 
of acceptable practice and managed towards those expectations. There are many essential components of inclusive education that are worth considering when implementing system improvement. This study surfaced many of those components that are critical considerations, including aligned leadership, establishing a culture of inclusion, and structures of support (grouping several high-frequency components).

Aligned Leadership. Aligned leadership horizontally and vertically is essential to improving inclusive education for students with ID. Aligned leadership was discussed most frequently of the coded components of inclusive education. The details in the results section highlighted reasons this was the case. Principals in all three districts described needing backing from the district office leadership, human resources, special education, teaching and learning, and the superintendent's office for a variety of reasons. Aligned leadership is more than agreeing to a shared vision. The implications of the essential nature of aligned leadership should inform the reader of a pathway to explore if interested in improving inclusive practices.

Aligned leadership horizontally and vertically in the system is an important finding of this study because of the implications this component has for future practice. While Loreman's (2007) Pillar 3 indicates that shared leadership is important at the building level, this study suggests that sustained improvement in inclusive education also needs the larger system's support. For example, in District 3, a larger district, they are systematically piloting inclusive practices in one of their "feeders" (several elementary and middle schools that feed into one of their four high schools) to 
intentionally scale up inclusive practice. Leadership at the school and district level described the importance of a commitment to inclusive practices from the school board, the superintendent, and senior cabinet leaders. The implications of this finding suggest strongly that moving away from segregated settings and improving inclusive practices will not happen in a vacuum. Sustained improvements in inclusive practice require a whole-system approach with building-level supports for principals to support effective implementation.

Segregated education for students with ID is an equity problem that can be addressed systematically. Through long range planning, public engagement and the organization's strategic priority process, a comprehensive plan can be developed with all levels of stakeholder engagement. Consider including formally the district's educational equity advisory committee, when it is formed (Oregon School Board Association, 2021). This group can help each district examine equity challenges the district is facing, and if segregated placements for students with disabilities is not meeting desired outcomes (see Table 1), this group can advise the superintendent and school board through their long-term planning and strategic priority process to address the problem of disproportionately segregated placements for students with ID. Part of a comprehensive plan to address educational disparities should include clear methods to continually monitor success of all improvements with clear deliverables and measurable objectives. It is recommended too, that prior to proceeding with implementation of a comprehensive plan for improving inclusive education, that engaging with state-level 
leadership on the legal merits of the plan to assure compliance with the IDEA, state administrative rules, and to seek perspective on possible unintended consequences of making dramatic departures from historical practices.

While the findings of this study suggest aligned leadership throughout the organization, the framework of implementation science suggests a collaborative approach with the implementers of this improvement effort (SISEP, 2020). Connecting to a limitation of this study, teachers were not interviewed, therefore it is not clear how these districts may have engaged in collaboration with teachers and teacher leaders with decision making around implementation of inclusive practices. Utilizing tools from implementation science in the decision-making phase, for example, the hexagon tool (NIRN, 2020), can support a true collaborative effort with teachers and specialists to examine the advantages, disadvantages and their role in the proposed implementation of an improvement such as inclusive practices as part of the effort toward aligned leadership. This collaborative effort is predicted to go a long way to securing the allimportant buy-in by implementers who, if participating in the change effort decisions, would see themselves as an important part of the solution.

Establishing a Culture of Inclusion. Leaders interviewed in this study were adamant that a common culture centered around belonging and normalizing a wider range of learners in each classroom sets the tone for all involved. Culture impacts how students feel and can support teachers who feel unsure or uncomfortable with welcoming learners with significant challenges into their classrooms. Culture seems to 
be the foundation on which all the other efforts to improve inclusion rest upon and connect. Education is a human business where everyone's thoughts, feelings and experiences shape the collective whole. When the leadership continually reinforces that all students belong in every space, that goes a long way to push back against many of the naysayers and barriers that come with equity work.

Roles and role clarity connect to culture, and ownership of student learning. The Principal in district 3 highlighted the importance of "whose" kids kept being referred to (often educators refer to a student as "belonging" to a specific case manager, for example, "oh, that is one of Michael's students," referring to the fact that Michael is the case manager). When the principal noticed that the staff moved from calling the students as, "hey, they are one of [enter case manager's name]'s kids" to "our kids," it was an indicator of a significant shift. This principal connected this notion of roles and ownership to work they had been doing in the building around asset-based language, which moves the culture and tone away from ableist thinking and deficit ideology.

Natural supports come naturally when the culture is about everyone belonging. Natural supports are when peers support a friend who may be having a rough day or needs some additional help or grace. This could be with a disability or an awkward social situation. The principals interviewed in this study spoke at length to example after example of how the culture of their inclusive schools increased students helping each other out and a decrease in bullying behavior. 
The implications of culture for leaders interested in inclusive education are that it is an essential part of the work and must be planned, monitored and sustained. Leaders interviewed for this study described the importance of shaping the narrative and highlighting the successes on a continual cycle. Communicating the positives of the inclusive setting with highlights going out in family/ community newsletters to videos being shot by students to celebrate the feeling of belonging. Culture should be considered when thinking of all aspects of the operation of a school, from transportation and bussing, to inclusive sporting events such as access to sports and considering Unified Sports as a way to invite all learners in, for example. Even examine food service settings and other areas where we can improve a sense of belonging and caring. One special education director interviewed suggested an indicator of success for that district is when their intramural sports roster matches the demographics of the district. They measure their success on how included all students are in all aspects of student learning, growth and development. Culture is key to leading this work and it cannot be ignored or underestimated.

Structures of Support. Structures of support refers to a grouping of components including training educators for inclusive practices, strategic teaming and shaping educator roles. These structures form the backbone of logistics when preparing a school or district for improving inclusive practices. Training for inclusive practices must be a priority and integrated with the professional development the district is offering as addressing priorities. Training should be ongoing and have clear objectives informed by 
assessment of system challenges and barriers. Training and professional development should also have a dedicated budget to support the work and have clear objectives informed by assessment of system challenges and barriers. For example, if general education teachers are unsure of how to provide access to a lesson for a particular student, could there be ongoing coaching to support that teacher with lesson design using UDL principles. Training needs to address all aspects of service delivery in inclusive settings with specialists and general education teachers alike.

Strategic teaming is essential for supporting all involved in delivering inclusive practices. Teaming from a leadership perspective is important because even leaders need to feel the support of leaders above, below and besides them (connects to aligned leadership). Grade level colleagues participating in teams is important to support one another's roles. Principals described many examples of how grade level and content area teams with specialists are critical to inclusive practice. In such teams, the general education teacher grew into the content expert and the specialist grew into the access expert- working together, they supported the implementation work. Teaming for problem solving is a key aspect of supporting the work. Leaders successfully improving inclusive practices anticipate teachers will need planning and problem solving time and schedule accordingly.

Role clarity is mentioned throughout this study because it is important for every staff member to understand how the work they are being asked to perform is essential for contributing to and supporting inclusion. According to the results of this study, 
leaders interested in inclusion work would be well advised to pay attention to roles and be mindful of areas where a lack of role clarity can impede efforts for inclusion.

School funding in Oregon can be a complex barrier to overcome. One district leader interviewed as part of this study described the funding challenges in Oregon, compared to other states. Oregon's funding levels are predictably discrepant from what school districts need in order to achieve equitable outcomes (Quality Education Commission, 2020). In order for a district to improve inclusive practices, districts must prioritize and commit funding to increasing resources critical to improving inclusive education practices within the scope of their allocated resources. Maintenance of effort (MOE) is a federal rule that is challenging for districts to stay in compliance within Oregon school funding. MOE is in place to protect special education funding, and the management part in Oregon is a dynamic when funding ebbs and flows (Oregon is an income tax-driven funding model and when a recession hits, schools are impacted); districts cannot increase in special education because under MOE rules, without exceptions, districts cannot reduce budgets for special education services when the inevitable recessions come about and districts have to reduce spending.

While school funding problems can be a significant barrier, there is a nuanced observation in this study that school funding challenges do not preclude inclusive practices in committed school districts. This study posits inclusive practice is more about a districts' willingness to include all students than solely about a lack of fiscal resources. The case to this point is District 1 . This small $(1,750$ total enrollment), rural district 
without significant local levy dollars, is able to decrease segregated settings and increase inclusive practices because it is a priority of the district. The school community and leaders are on board, they have created a culture of belonging, and improvement happens each and every day with all staff, all departments. District 1 is a notable district because of the laser-like focus the district has on the success of every single student, even those experiencing significant disabilities such as ID, autism, and behavioral disabilities.

Complex systems can change when there is the will to do so. For example, after September $11^{\text {th }}, 2001$ when the United States was attacked, the vulnerability of the safety of air travel was suddenly revealed. The country immediately prioritized the will to shore up safety screening for air travel, and the Transportation Safety Administration was established. Almost overnight, a complex system was vastly improved because of the will to prioritize the need for change. The implication is that we continue to segregate students with ID because many schools are willing to accept the disproportionately negative outcomes of segregated education, when the research is clear that inclusive practices predict improved outcomes (Andresen \& Nord, 2020; Ballard \& Dymond, 2018; C. Cole et al., 2004; S. M. Cole et al., 2020; Cosier et al., 2013; Dessemontet et al., 2012; Ryndak, Alper, Ward, Storch \& Montgomery, 2010; Ryndak, Morrison \& Sommerstein, 1999; Vinodrao, 2016). 


\section{Loreman's Pillars of Inclusion (Research Question Two)}

This study addresses the question of the relationship between Loreman's (2007) Pillars and components of inclusive education. The major findings (aligned leadership, culture and structure of support) emerged as clear and distinct themes through analysis. There were many overlapping themes when compared to the pillars, and the components found in this study support and bolster Loreman's Seven Pillars. Given that the study design was focused on school leaders, it is not surprising to see that the frequency of codes that fell under Pillar 2, Policy \& Leadership was the highest (see Figure 8). It was also not surprising to see many of the components that had emerged from coding and analysis had significant co-occurrences within each of the pillars when compared.

What was surprising was the emergent themes that did not quite fit or were variations of Loreman's (2007) Pillars, and are considered by leaders doing this work to be essential, based on their description. This study goes into much more detail as to some of the more subtle nuances and finds there are areas that were not part of the Pillars. These emergent themes and components are extensions on the theoretical framework and add value to the foundational pieces described. For example, the finding of aligned leadership as the most frequently coded component, was notably a different view from Loreman's description of policy and leadership. Loreman described the nature of shared leadership to support inclusive education in, "assisting in the acceptance of an inclusive approach by all members of the school community" (p. 26). 
He posited leadership at the school level and empowering teachers as essential in creating a culture that supports inclusive practice. When describing the relationship between policy and leadership, Loreman emphasized the need for leaders to believe in the work of inclusion, noting that inclusion is more than policy implementation and following a checklist. The major finding of aligned leadership suggests that improvement toward inclusive education cannot happen in a vacuum- it is necessary to have the full support of the community and leaders both horizontally and vertically.

Leaders interviewed in this study described their philosophical approach was tp adapt the system, or schools to the individual needs of the student (as opposed to an approach where the student needs to adapt to the system). Loreman's (2007) third pillar, School and Classroom Processes, grounded in research based practice connects directly to the finding of Structures of Support. In his third pillar, Loreman described the relationship between the purpose and function of a school, the beliefs of the staff, and efforts to adapt the school to the student:

If schools truly believe that they are there to meet the educational, emotional, social and other needs of children then it stands to reason that they need to be willing to change and adapt to suit these individuals rather than the other way around. (p. 27)

The finding in this study of Structures of Support refer to efforts leaders make to, as Loreman pointed out, adapt the school to the student. Through training, teaming and addressing adaptive changes to educator roles in a model of inclusive practice, these efforts lead to sustained change, or adaptation to a climate that supports individual 
student learning needs. This is another example of how leaders came to the same conclusion as Loreman's theoretical framework for inclusive education.

The implication and importance of orienting to a mindset of school adapting to the student is for leaders to consider the notion of what the target of improvement should focus upon. This orientation is juxtaposed to the notion that if the student is not succeeding, therefore something must be wrong with them. This notion gives a clue on how to directly address deficit ideology and thinking; leaders must lead with the message that the system is not working for every student, hence the effort for improvement is in how schools operate and teach students with a wide range of learning needs.

An interesting aspect of these findings related to the pillars is the importance of considering educator roles in light of improving inclusive education. Broadly speaking, the need to address educator roles is set in the historical context of how teachers and specialists have operated within a segregated education system. This connects to the notion that the IDEA is based on the presumption of a medical model where certain expertise is needed to teach students with disabilities and to how specialists are prepared in to serve in these roles. This component was outside of the theoretical frames used, yet each of the districts examined identified the need to address the dynamic of educator roles.

Special education teacher preparation has moved firmly to prepare and ground aspiring practitioners in alignment with a strong core curriculum (Leko et al., 2015); yet 
when they arrive at their new jobs, in most cases they are asked to perform a role consistent with a traditional service delivery model within segregated school settings (Figure 1). It stands to reason, an adaptive shift must be made intentionally for existing specialists and generalists to see their role in a different light with respect to service delivery in an inclusive education model, who should provide that service, and where should that service be delivered.

Reshaping educator roles has significant implications for how leaders think about this work. Educators in practice today (generally speaking) were trained in the context of a segregated system of education for students with significant disabilities. The implication of paying attention to these shifting roles is that leaders must be mindful that the ask of these educators is to practice differently and evolve how they do their work, which may be challenging for practitioners who already are fatigued by the expected cycles of initiative overload; inclusive practices may very well be seen as one more thing that will go away again after a while like many educational innovations.

The field of special education has many specialists where those professions and practices must also evolve to contribute meaningfully toward inclusive practices. Related services professionals are one example; many districts used to hire adaptive PE teachers- we might now think how can the regular education PE teacher be supported to adapt their instruction to students with mobility barriers. A physical therapist is able to do this and the mindful leader will see time and energy will need to go into planning and support for both professionals. School psychology is another area where the role 
moves from a traditional model of utilizing their skills as assessors only and may shift to utilizing their expertise in application of supporting teams with functional behavior assessments in the context of general education and point of performance coaching to support behavior, for example.

Operational efficiency is another important finding that extends the notion of Training \& Resources, Pillar 7 in Loreman's (2007) framework. The example of organizational efficiencies discussed in chapter 4 is an example of leadership that by virtue of their leadership practices, District 1 is able to keep their leaders focused on their priorities and thus partly addressing the question of inadequate resources as a barrier to inclusion. In other words, by virtue of leadership practices and connecting district (system) priorities, leaders are able to do the work of inclusion because it is their job and they are accountable to the results. There is permission to not take on any more projects or initiatives if they do not align with the top five priorities of the district (one of them happens to be inclusive practice).

There are implications for school and district leaders in practices other than improving inclusive education. Oregon educational leaders are beyond busy and do not have time for everything they are asked to do to lead and manage within their areas of responsibility. With so many competing initiatives, it is necessary that leaders prioritize what is most important and focus on leading those improvement efforts. The implication is that school and district leaders would benefit by recognizing that the cycle of innovation, when not directly aligned with district priorities, can be a significant 
distraction. The aforementioned example of District 1 where they practice tight leadership structures with "no new shiny things", is an example of this kind of discipline. When one of the district/ board priorities are to include all learners in rigorous learning, it can free leaders up in the organization to lead the right work.

While it is clear that leader perspective has added deeper and nuanced findings in addition to Loreman's (2007) pillars, this study has a significant limitation in that teachers were not interviewed. Teacher voice is necessary to surface their perceptions of what structures and supports would need to support growing in inclusive practices. While leaders' perceptions about structures as they are leading the work of improving access to the general education curriculum for all learners is a great starting point, because teacher voice is missing from these findings to check leader assumptions and observations, this presents a threat to validity.

\section{Educator Attitudes and Ableism (Research Question Three)}

Educator attitude is described by leaders as one of the most important considerations to address when improving inclusive practices. While leaders said they did not address ableism directly, each leader affirmed the presence of ableism in educational settings and a barrier to the work of inclusive practices. Leaders described the tension between either evolve the thinking or find another place to work. A principal described:

I could not do it without the mindset of our teachers. And I will say that we had almost $100 \%$ turnover in the 3 years that I was implementing this which needed to happen anyway. [the message is] ... this is the work we're going to do, come alongside us and let's do it. You're supported. But there's some people who 
didn't believe in it. And thankfully they saw that this is what we're doing and they moved on. And there's a lot of other factors.

A special education director further elaborated on this dynamic:

... you know your challenges with this concept. And at the same time ... if this isn't the right district for you, that's okay because here's what we're committed to doing, and so you can you can decide . . . it has been difficult, especially the first couple of years . . . the wave of the wave of resistance was really high in the beginning, but then just over time it was like, oh, they're not going to relent on this.

As pointed out by another principal:

Their attitude ... is getting in the way of, of our students having the level of success they need so I do think it's super important. And as the principal my job is to gently help and guide that adult to move their thinking. Not come at it from a negative, [but] really listen to them and understand what is it about their role. That is having them make these decisions, but ultimately we do need to help our learning specialist see that they are providing a service, and in pursuit of having all children have the access.

While Avramidis and Norwich (2002) outlined child-related variables, educational

environment-related variables, and teacher-related variables, the leaders interviewed in this study addressed all of these through intentional yet indirect ways. Child Related Variables include teacher perception of the child and their disabling condition as an area to be a factor influencing teacher attitude toward inclusive education for students with disabilities. In the design for this study, questions were included in the interviews designed to understand how leaders thought about the role of educator attitude as it relates to moving toward inclusive practice. Leaders described that while attitudinal barriers exist in their system, the most commonly identified way to address attitudinal barriers to inclusive education is through structural and system supports. 
Leaders described fear as being central to attitudinal resistance, in their observation. One principal describes a teacher overheard saying, "I've never had a student with Down Syndrome and my class I have no idea what to do with them." Avramidis and Norwich's (2002) frame would suggest that positive experiences would change the dynamics. Leaders in this study addressed the need in the same fashion. Implications for leaders is that if a teacher were coming from a place of fear around this dynamic, how might that leader facilitate a positive experience for that teacher? An example is to allow that teacher to visit classrooms where students with ID are learning alongside their peers, interacting with them and having positive experiences. Or doing a learning walk in an inclusion classroom in another building or district to expand their view of what is possible.

Educational Environment is about a supportive school setting that contributes to a positive attitude about inclusive education. Structures of support and a culture of inclusion, both components and major findings in this study, directly address this element of Avramidis and Norwich's (2000) framework and impact educator attitude directly. The implication is that leaders interested in improving inclusive education will address attitudinal barriers and resistance by supporting the educational environment through training, supporting roles and strategic teaming.

Creating a culture of inclusion in a school environment is an essential component of moving away from segregated classroom settings and toward inclusive practices. Leaders described how they intentionally messaged a narrative of inclusive education, 
emphasizing successes and overcoming challenges. Leaders described the impact of how changing culture normalizes educating all students in the general education setting and that culture supports staff when situations become difficult. Examples were provided of principals supporting educators attitudinal shifts by supporting their skill development and building on existing skills. Leaders described a small percentage of teachers or specialist leaders who simply were not interested in making change to their practices and were supported with a decision to move onto a district that would be a better fit.

A significant limitation to this study, in particular when addressing attitudinal barriers, is the absence of teacher and specialist voice. Therefore, it is noted that the findings about educator attitudes are based on the observations and interactions by school and district leaders. It is important to note that while attitudinal barriers were absolutely described by administrators, this study lacks a direct examination of teacher attitudinal barriers. The dynamic is presented in this study as a presumptive resistance to change in the part of teachers and specialists. The lack of direct verification is a clear path for further investigation as an interview with teachers would have supported this validity weakness and suggest stronger generalizability.

\section{Situating This Study in the Larger Context and Implications for Practice}

The purpose of this study is to understand how school and district leaders described their thinking, planning and actions in order to improve inclusive practices for students with ID. To this aim, that goal was reached and the findings present a deeper understanding and working knowledge of the theoretical frameworks presented to 
address placement by creating more opportunities for inclusion and truly moving away from segregated settings as a default placement for students with ID.

Using disability studies as a theoretical framework to understand the impact of ableism and deficit ideology helped to understand the barrier of attitudinal variables and practice of inclusive education. The connection between deficit ideology and ableism form assumptions about placement and expectations for growth and capability of students with ID is supported with the findings of this study. Every educational leader interviewed in this study described significant attitudinal barriers to the work of improving inclusive practices and affirmed the connection between attitude and the persistence of segregated settings. Avramidis and Norwich (2002) outlined variables to examine and leaders in this study shared their insights on addressing these barriers. Loreman's (2007) pillars have stood the test of time as guideposts for inclusive education for leaders to pay attention to. This study affirmed the importance of those pillars and surfaced components that expand on the pillars to bring depth and more considerations for leaders to think about.

There were aspects of responses from these leaders that highlighted other aspects of improving inclusive practice suggesting opportunities for further research, such as restructuring roles, a focus on adapting the system to fit the learner and leadership practices that prioritize inclusive education so it is not just one more thing for a school leader to be responsible for. The insight about roles highlights the importance of teacher and specialist preparation programs given the pressures for districts to 
achieve improved outcomes for students with ID when inclusive practice is clearly a way to address many of the problems districts are facing in light of Lane v. Brown (2016) and Endrew F. v. Douglas County School District (2017). Teacher and specialists preparation programs should consider how to best prepare practitioners to see their roles as evolving in an ever-changing range of practices when they start work in the field of education.

In the past 6 years, schools in Oregon are compelled to stop training people with significant disabilities to do work in sheltered workshops (Lane v. Brown, 2016) and must improve programs in order to prepare students for meaningful, engaged, independent life after they leave their school experience. The IDEA encourages but does not require schools to include all learners in the classroom. In fact, the IDEA compels schools to have a range of options- including segregated settings. Moving toward inclusive practices, while not required, is an evidence-based pathway to improve programs and outcomes. It is, however, not an easy path. School districts in Oregon are inadequately funded (Quality Education Commission, 2020) to do the work that creates opportunities for all students. It will take leadership from within this context to transform our schools to meet these challenges.

The major findings of this study (aligned leadership, culture, structures, and how those structures indirectly address attitude) can provide leaders interested in improving inclusive practices insight as to their assessment of their schools and districts as to what needs to be in place, and inform consideration of long-range and strategic planning. For 
example, if a special education director wants to address the district's B5 target (too much segregation), consideration of aligned leadership might lead to an assessment of the school board, superintendent, teaching and learning director, and school principals of where their thinking is on the subject before proceeding with improvement toward inclusive practices. Additionally, that director may consider that if there is not aligned leadership on board with improving inclusive practices, and placements start to change before the general education system is ready, the resistance from general education teachers will be well-founded. A principal may want to improve inclusive practices in their school and these findings may help shape their thinking about the district supports they would need should fiscal needs emerge or the community takes issue with the changes.

Recommendations include supporting principal leadership development and regional collaboration and learning walks alongside other schools improving inclusive practice. Inclusive practices happen between a teacher and a student, and the instructional leader most positioned to lead this work, is the school principal. From a communications standpoint, as part of a plan for including students with ID in the general education, it is recommended that each district and school community develop and broadcast an intentional narrative on why inclusive practices, highlighting the success stories to reinforce the benefits of moving towards improving inclusion. Structures need to be supported such as utilization of existing resources such as related services, assistive technology and the consideration of natural peer supports. A 
recommendation for further research is that a formal action research is conducted with the partnership of a local university during the planning and implementation phases of the improvement process of a district undertaking an initiative to implement inclusive practices. A partnership such as this can go a long way to reducing the research to practice gap and bring significant value to both organizations for the benefit of students.

In June of 2021, the Oregon House passed Senate Bill 732 that requires school districts in Oregon to create an educational equity advisory committee with representatives from the community, school board, and school staff. This committee is charged with advising school boards on how decisions affect student equity and to "be a resource [to superintendents and school boards] when events may negatively impact historically underrepresented students" (Oregon School Board Association, 2021). This committee may be an entry point to identify that the disproportionate and segregated settings for students with ID is an equity problem. Intersecting with the SB 732 committee may be the accountability for a district to address the B5 indicator as well as the school district's responsibility for targets in Post-School Outcomes participation and data.

A significant limitation of this study, that only leaders were interviewed, presents an opportunity for further research with the addition of teacher, specialist, parent and student voice. Interviews exploring similar research questions examining perceived attitudinal barriers and understanding perspective from these stakeholders would add 
significant depth and dimension to these findings. Interviewing these stakeholders would verify assumptions made and control the threats to validity that currently exist.

What started out as an exploration in order to understand a problem of practice, has become a source of incredible learning, personal passion and a sense of optimism. The learning from colleagues is remarkable (really, an honor), and the process by which to surface their insights and bold leadership has been a deep journey on how to truly understand a phenomena within a complex system. To look to the wisdom and practices of leaders who have led down the path of improving systems that lead to equitable outcomes has been a tremendous gift. And yet, the reality is that many educators today believe a student experiencing ID should be taught out in the portable classroom with the other "life skills kids." There are, of course, many examples where this is not the case, but those are the exceptions. When students experiencing ID are segregated and not allowed to fully participate because of antiquated systems and ableist attitudes, society as a whole loses out because their valuable contributions will not have been realized, and the right to fully participate has been shut down. Solving the problem of segregated settings for students with ID will not happen by accident. It will take a clear commitment by communities, school and district leaders, and educators to make it a priority that improved outcomes for all students is import and worthy of attention. 


\section{References}

Agmon, M., Sa'ar, A., \& Araten-Bergman, T. (2016). The person in the disabled body: A perspective on culture and personhood from the margins. International Journal for Equity in Health, 15(1), 147.

Agran, M., Jackson, L., Kurth, J. A., Ryndak, D., Burnette, K., Jameson, M., Zagona, A., Fitzpatrick, H., \& Wehmeyer, M. (2020). Why aren't students with severe disabilities being placed in general education classrooms: Examining the relations among classroom placement, learner outcomes, and other factors. Research and Practice for Persons with Severe Disabilities, 45(1), 4-13. https://doi.org/10.1177/1540796919878134

Aguilar, E. (2020). Coaching for equity: Conversations that change practice. John Wiley \& Sons.

Ainscow, M., Farrell, P., \& Tweddle, D. (2000). Developing policies for inclusive education: a study of the role of local education authorities. International Journal of Inclusive Education, 4(3), 211-229. https://doi.org/10.1080/13603110050059150

Altermark, N. (2014). The ideology of neuroscience and intellectual disability: Reconstituting the "disordered" brain. Disability and Society, 29(9), 1460-1472, https://doi.org/10.1080/09687599.2014.953244

Annamma, A., Connor, D., \& Ferri, B. (2013). Dis/ability critical race studies (DisCrit): theorizing at the intersections of race and dis/ability. Race Ethnicity and Education, 16(1), 1-31. https://doi.org:10.1080/13613324.2012.730511

Andresen, J. M., \& Nord, D. (2020). Cognitive disability and postsecondary education: A national study on earnings. Developmental Disabilities Network Journal, 1(1), Article 5. https://doi.org/10.26077/bs8g-m969

Andrews, J. E., Carnine, D. W., Coutinho, M. J., Edgar, E. B., Forness, S. R., Fuchs, L. S., Jordan, D., Kauffman, J. M., Patton, J. M., Paul, J., Rosell, J., Rueda, R., Schiller, E., Skrtic, T. M., \& Wong, J. (2000). Bridging the special education divide. Remedial and Special Education, 21(5), 258-267.

Artiles, A., Kozleski, E., Trent, S., Osher, D., \& Ortiz, A. (2010). Justifying and explaining disproportionality, 1968-2008: A critique of underlying views of culture.

Exceptional Children, 76(3), 279-299. 
Avramidis, E., \& Norwich, B. (2002). Teachers' attitudes towards integration/inclusion: A review of the literature. European Journal of Special Needs Education, 17(2), 129-147. https://doi.org/10.1080/08856250210129056

Baglieri, S., Bejoian, L. M., Broderick, A. A., Connor, D. J., \& Valle, J. (2011). [Re]Claiming "inclusive education" toward cohesion in educational reform: Disability studies unravels the myth of the normal child. Teachers College Record, 113(10), 21222154.

Baglieri, S., Valle, J. W., Connor, D. J., \& Gallagher, D. J. (2011). Disability studies in education: The need for a plurality of perspectives on disability. Remedial and Special Education, 32(4), 267-278. https://doi.org/10.1177/0741932510362200

Ballard, S. L., \& Dymond, S. K. (2018). Inclusive education for secondary age students with severe disabilities and complex health care needs. Intellectual and Developmental Disabilities, 56(6), 427-441. https://doiorg.proxy.lib.pdx.edu/10.1352/1934-9556-56.6.427

Banks, L., \& Polack, S. (2014). The economic costs of exclusion and gains of inclusion of people with disabilities. http://disabilitycentre.Ishtm.ac.uk/new-reporteconomic-costs-exclusion-gains-inclusion-people-disabilities/

Bateman, D., \& Bateman, C. F. (2014). A principal's guide to special education (3rd ed.). Council for Exceptional Children.

Bogart, K. R., \& Dunn, D. S. (2019). Ableism special issue introduction. Journal of Social Issues, 75(3), 650-664. https://doi.org/10.1111/josi.12354

Boston-Kemple, T. E. (2012). A conceptual analysis of key concepts in inclusive education (Doctoral dissertation, University of lowa). lowa Research Online. https://ir.uiowa.edu/etd/2828

Bouck, E. C. (2012). Secondary students with moderate/severe intellectual disability: Considerations of curriculum and post-school outcomes from the National Longitudinal Transition Study-2. Journal of Intellectual Disability Research, 56(12), 1175-1186.

Bouck, E. C., \& Joshi, G. S. (2016). Transition and students with mild intellectual disability: Findings from the National Longitudinal Transition Study-2. Career Development and Transition for Exceptional Individuals, 39(3), 154-163. https://doi.org/10.1177/2165143414551408

Bourgeault, I., Dingwall, R., \& De Vries, R. (2010). The SAGE handbook of qualitative methods in health research. Sage. 
Boyle, C., Topping, K., \& Jindal-Snape, D. (2013). Teachers' attitudes towards inclusion in high schools. Teachers and Teaching: Theory and Practice, 19(5), 527-542. https://doi.org/10.1080/13540602.2013.827361

Brantlinger, E., Jimenez, R., Klingner, J., Pugach, M., \& Richardson, V. (2016). Qualitative Studies in Special Education. Exceptional Children, 71(2), 195-207. https://doi.org/10.1177/001440290507100205

Brock, M. (2018, July) Trends in the educational placement of students with intellectual disability in the United States over the past 40 years. American Journal on Intellectual and Developmental Disabilities, 123(4), 305-314.

Browder, D. M., Jimenez, B. A., Mims, P. J., Knight, V. F., Spooner, F., Lee, A., \& Flowers, C. (2012). The effects of a "tell-show-try-apply" professional development package on teachers of students with severe developmental disabilities. Teacher Education and Special Education, 35(3), 212-227. https://doi.org/10.1177/0888406411432650

Brown v. Board of Education, 347 U.S. 483 (1954)

Brown, Z. (2016). Inclusive education: Perspectives on pedagogy, policy and practice. Routledge.

Bryk, A., Gomez, L., Grunow, A., \& LeMahiew, P. (2017). Learning to improve: How America's schools can get better at getting better (5th ed.). Harvard Education Press.

Cambridge-Johnson, J., Hunter-Johnson, Y., \& Newton, N. G. L. (2014). Breaking the silence of mainstream teachers' attitude towards inclusive education in the Bahamas: High school teachers' perceptions. The Qualitative Report, 19(42), 1-19.

Campbell, F. A., \& Kumari, F. (2008). Exploring internalized ableism using critical race theory. Disability and Society, 23(2), 151-162. https://doi.org/10.1080/13668250310001616407

Campbell, J., Gilmore, L., \& Cuskelly, M. (2009). Changing student teachers' attitudes towards disability and inclusion. Journal of Intellectual and Developmental Disability, 28(4), 369-379. https://doi.org/10.1080/13668250310001616407

Carter, E. W., \& Hughes, C. (2005). Increasing social interaction among adolescents with intellectual disabilities and their general education peers: Effective interventions. Research and Practice for Persons with Severe Disabilities, 30(4), 179-193. https://doi.org/10.2511/rpsd.30.4.179 
Carter, E. W., \& Hughes, C. (2006). Including high school students with severe disabilities in general education classes: Perspectives of general and special educators, paraprofessionals, and administrators. Research and Practice for Persons with Severe Disabilities, 31(2), 174-185. https://doi.org/10.1177/154079690603100209

Castaneda, R., \& Peters, M.L. (2000). Ableism. In M. Adams, W. J. Blumenfled, R. Castaneda, H. W. Hackman, M. L. Peters, \& X. Zuniga (Eds.), Readings for diversity and social justice (pp. 319-323). Routledge.

Cole, C., Waldron, N., \& Majid, M. (2004). Academic progress of students across inclusive and traditional settings. Mental Retardation, 42(2), 136-144. doi: 10.1352/0047-6765(2004)42

Cole, S. M., Murphy, H. R., Frisby, M. B., Grossi, T. A., \& Bolte, H. R. (2020). The Relationship of Special Education Placement and Student Academic Outcomes. The Journal of Special Education. https://doi.org/10.1177/0022466920925033

Connor, D. J., \& Ferri, B. A. (2005). Integration and inclusion-A troubling nexus: Race, disability, and special education. The Journal of African American History, 90(1/2), 107-127. https://doi.org/10.1086/JAAHv90n1-2p107

Cosier, M., Causton-Theoharis, J., \& Theoharis, G. (2013). Does access matter? Time in general education and achievement for students with disabilities. Remedial and Special Education, 34(6), 323-332.

Craig, S., Smith, S., \& Frey, B. (2019). Professional development with universal design for learning: Supporting teachers as learners to increase the implementation of UDL. Professional Development in Education, 1-16. https://doi.org/10.1080/ 19415257.2019.1685563

Davis, L. J. (2017). The disability studies reader (5th ed.). Routledge.

Dessemontet, R., Bless, G., \& Morin, D. (2012). Effects of Inclusion on the academic achievement and adaptive behaviour of children with intellectual disabilities. Journal of Intellectual Disability Research, 56(6), 579-587. https://doi: 10.1111/j.1365-2788.2011.01497.x

Dessemontet, R., \& Gless, G. (2013). The impact of including children with intellectual disability in general education classrooms on the academic achievement of their low-, average-, and high-achieving peers. Journal of Intellectual and Developmental Disability, 38(1), 23-30. https://doi.org/10.3109/13668250.2012.757589 
Disabled World. (2019) blog. Sheltered Workshops: History and Definition. https://www.disabled-world.com/definitions/sheltered.php

Donovan, M. S., \& Cross, C. T. (Eds.). (2002). Minority students in special and gifted education. National Academies Press.

Endrew F. v. Douglas County School District Re-1, 137 S. Ct. 988 (2017).

Education for All Handicapped Children Act of 1975, Pub. L. No. 94-142 (1975).

Ferguson, P., Ferguson, D. L., \& Brodsky, M. M. (2008). Away from the public gaze: A history of the Fairview Training Center and the institutionalization of people with developmental disabilities in Oregon. Monmouth OR: Western Oregon University, Teaching Research Institute.

Fierros, E. G., \& Conroy, J. W. (2002). Double jeopardy: An exploration of restrictiveness and race in special education. In D. J. Losen \& G. Orfield (Eds.), Racial inequity in special education (pp. 39-70). Harvard Educational Press.

Fisher, M., \& Meyer, L. (2002). Development and social competence after two years for students enrolled in inclusive and self-contained educational programs. Research and Practice for Persons with Severe Disabilities, 27(3), 165-174.

Fixsen, D. L., Naoom, S. F., Blase, K. A., Friedman, R. M., \& Wallace, F. (2005). Implementation research: A synthesis of the literature. The National Implementation Research Network.

Forlin, C., \& Chambers, D. (2011). Teacher preparation for inclusive education: Increasing knowledge but raising concerns. Asia-Pacific Journal of Teacher Education, 39(1), 17-32.

Frattura, E., \& Capper, C. A. (2006). Segregated programs versus integrated comprehensive service delivery for all learners: Assessing the differences. Remedial and Special Education, 27(1), 355-364.

Frey, B. (2018). The SAGE encyclopedia of educational research, measurement, and evaluation (Vols. 1-4). Sage. https://dx.doi.org/10.4135/9781506326139

Fuchs, L. S., Fuchs, D., Compton, D. L., Wehby, J., Schumacher, R. F., Gersten, R., \& Jordan, N. C. (2015). Inclusion versus specialized intervention for very-lowperforming students. Exceptional Children, 81(2), 134-157.

Gargiulo, R. M., \& Metcalf, D. J. (2017). Teaching in today's inclusive classrooms : a universal design for learning approach ( $3^{\text {rd }} \mathrm{ed}$.). Cengage Learning. 
Gilmore, L., Campbell, J., \& Cuskelly, M. (2010). Developmental expectations, personality stereotypes, and attitudes towards inclusive education: Community and teacher views of Down Syndrome. International Journal of Disability, Development, and Education, 50(1), 65-76.

Gilmour, A. F. (2018). Has inclusion gone too far? Weighing its effects on students with disabilities, their peers, and teachers. Education Next, 18(4), 8-16.

Gorski, P. C. (2011). Unlearning deficit ideology and the scornful gaze: Thoughts on authenticating the class discourse in education. Counterpoints, 402, 152-173.

Grindal, T., Schifter, L., Schwartz, G., \& Hehir, T. (2019). Racial differences in special education identification and placement: Evidence across three states. Harvard Educational Review, 89(4), 525-553. https://doi.org/10.17763/1943-504589.4.525

Hall, C. B. (2019). Training and supporting general education teachers to promote inclusive practices. ProQuest Dissertations Publishing.

Hamilton, L., Corbett-Whittier, C., Lagrange, M., Birch, J., \& Scott, Wendy. (2013). Using case study in education research. Sage.

Hehir, T. (2002). Eliminating ableism in education. Harvard Educational Review, 72(1), 1-32.

Heifetz, R. A., Grashow, A., \& Linsky, M. (2009). The practice of adaptive leadership. Harvard Business Press.

Helmstetter, E., Curry, C., \& Sampson-Saul, M. (1998). Comparison of general and special education classrooms of students with severe disabilities. Education And Training In Mental Retardation And Developmental Disabilities, 33(3), 216-227.

Hornby, G. (2014). Inclusive special education: Evidence-based practices for children with special needs and disabilities. Springer.

Horrocks, J. L., White, G., \& Roberts, L. (2008). Principals' attitudes regarding inclusion of children with Autism in Pennsylvania Public Schools. Journal of Autism and Developmental Disorders, 38(8), 1462-1473. https://doi.org/10.1007/s10803007-0522-x

Hurt, A. P. (2007). The impact of professional development on general-education teachers' attitudes toward the inclusion of students with disabilities in the inclusive environment (Doctoral dissertation, University of Washington). ProQuest Dissertations Publishing. 
Individuals with Disabilities Education Act of 2004, 20 U.S.C § 1400 (2004).

Jackson, L. B., Ryndak, D. L., \& Wehmeyer, M. L. (2008). The dynamic relationship between context, curriculum, and student learning: A case for inclusive education as a research-based practice. Research and Practice for Persons with Severe Disabilities, 34(1), 175-195. https://doi.org/10.2511/rpsd.33.4.175

Kame'enui, E., \& Simmons, D. (1999). Toward successful inclusion of students with disabilities: The architecture of instruction. Council for Exceptional Children.

Kim, J.-R. (2011). Influence of teacher preparation programmes on preservice teachers' attitudes toward inclusion. International Journal of Inclusive Education, 15(3), 355-377. https://doi.org/10.1080/13603110903030097

Kleinert, H., Towles-Reeves, E., Quenemoen, R., Thurlow, M., Fluegge, L., Weseman, L., \& Kerbel, A. (2015). Where students with the most significant cognitive disabilities are taught: Implications for general curriculum access. Exceptional Children, 81(3), 312-328. https://doi.org/10.1177/0014402914563697

Kress-White, M. (2009). The quest of inclusion: Understandings of ableism, pedagogy and the right to belong (Master's thesis, University of Saskatchewan). Harvest Electronic Theses and Dissertations. http://hdl.handle.net/10388/etd-09202009155437

Kurth, J. A., Born, K., \& Love, H. (2016). Ecobehavioral characteristics of self-contained high school classrooms for students with severe cognitive disability. Research and Practice for Persons with Severe Disabilities, 41(4), 227-243. https://doi.org/10.1177/1540796916661492

Lambrecht, J., Lenkeit, J., Hartmann, A., Ehlert, A., Knigge, M., \& Spörer, N. (2020). The effect of school leadership on implementing inclusive education: How transformational and instructional leadership practices affect individualised education planning. International Journal of Inclusive Education, 1-15. https://doi.org/10.1080/13603116.2020.1752825

Lane v. Brown, 166 F. Supp. 3d 1180 (D. Or. 2016).

Leko, M. M., Brownell, M. T., Sindelar, P. T., \& Kiely, M. T. (2015). Envisioning the future of special education personnel preparation in a standards-based era. Exceptional Children, 82(1), 25-43. https://doi.org/10.1177/0014402915598782

Loreman, T. (2007). Seven pillars of support for inclusive education: Moving from "why?" to "how?" International Journal of Whole Schooling, 3(2), 22. 
Maxwell, J. A. (2013). Qualitative research design: An interactive approach (3rd ed.). Sage Publications.

Mcdonnell, J., Thorson, N., \& Mcquivey, C. (2000). Comparison of the instructional contexts of students with severe disabilities and their peers in general education classes. Research and Practice for Persons with Severe Disabilities, 25(1), 54-58.

McKay, S. (2017) Quality improvement approaches: Implementation science. Carnegie Commons Blog. https://www.carnegiefoundation.org/blog/qualityimprovement-approaches-implementation-science/

McLeskey, J., \& Waldron, N. L. (2002). Professional development and inclusive schools: Reflections on effective practice. The Teacher Educator, 37(3), 159-172.

Merriam, S. B., \& Tisdell, E. J. (2016). Qualitative research: A guide to design and implementation (4th ed.). Jossey-Bass

Messinger-Willman, J., \& Marino, M. T. (2010). Universal design for learning and Assistive technology: Leadership considerations for promoting inclusive education in today's secondary schools. NASSP Bulletin, 94(1), 5-16. https://doi.org/10.1177/0192636510371977

Meyer, A., Rose, D. H., \& Gordon, D. (2014). Universal design for learning: Theory and practice. CAST Professional Publishing.

Monsen, J. J., \& Frederickson, N. (2004). Teachers' attitudes towards mainstreaming and their pupils' perceptions of their classroom learning environment. Learning Environments Research, 7(2), 129-142. https://doi.org/10.1023/

B:LERI.0000037196.62475.32

Morningstar, M. E., Kurth, J. A., \& Johnson, P. E. (2017). Examining national trends in educational placements for students with significant disabilities. Remedial and Special Education, 38(1), 3-12. https://doi.org/10.1177/0741932516678327

National Academies of Sciences, Engineering and Medicine. (2018). How people learn II: Learners, contexts, and cultures. The National Academies Press. https://doi.org/10.17226/24783

National Council on Disability. (2015). Breaking the school-to-prison pipeline for students with disabilities. https://www.ncd.gov/system/files_force/Documents/ NCD_School-to-PrisonReport_508-PDF.pdf?download=1

National Implementation Research Network (NIRN). (2020). Active implementation hub. https://nirn.fpg.unc.edu/ai-hub 
Navarro, S. B., Zervas, P., Gesa, R. F., \& Sampson, D. G. (2016). Developing teachers' competences for designing inclusive learning experiences. Educational Technology and Society, 19(1), 17-27.

Nishimura, T. (2014). Effective professional development of teachers: A guide to actualizing inclusive schooling. International Journal of Whole Schooling, 10(1), 19.

Olmstead v. L.C., 527 U.S. 581, 119 (1999).

Olson, A., Leko, M. M., \& Roberts, C. A. (2016). Providing students with severe disabilities access to the general education curriculum. Research and Practice for Persons with Severe Disabilities, 41(3), 143-157. (If remove, remove M. R. from other Olson??)

Olson, M. R., Chalmers, L., \& Hoover, J. H. (1997). Attitudes and attributes of general education teachers identified as effective inclusionists. Remedial and Special Education, 18(1), 28-35.

Oregon Department of Education. (2020). Annual report. Is title actually Oregon statewide report card 2019-2020?? https://files.eric.ed.gov/fulltext/ED612759.pdf

Oregon School Board Association. (2021). Retrieved from: http://www.osba.org/NewsCenter/Announcements/2021/20210614Committee.aspx - Did you retrieve this document: House endorses school district educational equity advisory committees - and add date retrieved if it could change

Padilla, A., \& Tan, P. (2019). Toward inclusive mathematics education: A metatheoretical reflection about countering ableism in mathematics standards and curriculum. International Journal of Qualitative Studies in Education, 32(3), 299-322. https://doi.org/10.1080/09518398.2019.1576941

President's Commission on Excellence in Special Education. (2002). A new era: Revitalizing special education for children and their families. U.S. Department of Education, Publications Center.

President's Committee on Mental Retardation. (1977). Mental retardation: Past and present.

Quality Education Commission. (2020). Quality education model. Final report. https://www.oregon.gov/ode/reports-and-data/taskcomm/Pages/ QEMReports.aspx 
Rainforth, B. (2000). Preparing teachers to educate students with severe disabilities in inclusive settings despite contextual constraints. Research and Practice for Persons with Severe Disabilities, 25(2), 83-91. https://doi.org/10.2511/ rpsd.25.2.83

Rauscher, L., \& McClintock, J. (1997). Ableism curriculum design. In M. Adams, L. A. Bell, \& P. Griffen (Eds.), Teaching for diversity and social justice (pp. 198-231). Routledge.

Riehl, C. J. (2009). The principal's role in creating inclusive schools for diverse students: A review of normative, empirical, and critical literature on the practice of educational administration. Journal of Education, 189(1-2), 183-197. https://doi.org/10.1177/0022057409189001-213

Rose, T. (2013). The Myth of Average. Retrieved from: https://www.youtube.com/watch?v=4eBmyttcfU4

Rose, T. (2016). The end of average: How we succeed in a world that values sameness (First edition.). HarperOne, an imprint of HarperCollins Publishers.

Rotatori, A., Obiakor, Festus E, \& Bakken, Jeffrey P. (2011). History of special education (1st ed., Advances in special education; v. 21). Bingley, U.K.: Emerald.

Ryndak, D., Alper, S., Hughes, C., \& Mcdonnell, J. (2012). Documenting Impact of Educational Contexts on Long-Term Outcomes for Students with Significant Disabilities. Education and Training in Autism and Developmental Disabilities, 47(2), 127-138.

Ryndak, D., Reardon, R., Benner, S. R., \& Ward, T. (2007). Transitioning to and Sustaining District-Wide Inclusive Services: A 7-Year Study of a District's Ongoing Journey and its Accompanying Complexities. Research and Practice for Persons with Severe Disabilities, 32(4), 228-246. https://doi.org/10.2511/rpsd.32.4.228

Ryndak, D., Morrison, A., \& Sommerstein, L. (1999). Literacy before and after Inclusion in General Education Settings: A Case Study. Research and Practice for Persons with Severe Disabilities, 24(1), 5-22.

Ryndak, D., Ward, T., Alper, S., Storch, J., \& Montgomery, J. (2010). Long-term outcomes of services in inclusive and self-contained settings for siblings with comparable significant disabilities. Education And Training In Autism And Developmental Disabilities, 45(1), 38-53.

Saldaña, J. (2021). The coding manual for qualitative researchers ( $4^{\text {th }}$ ed.). Sage. 
Sari, H. (2007). The influence of an in-service teacher training (INSET) programme on attitudes towards inclusion by regular classroom teachers who teach deaf students in primary schools in Turkey. Deafness Education International, 9(3), 131-146.

Sauer, J., \& Jorgensen, C. (2016). Still caught in the continuum: A critical analysis of least restrictive environment and its effect on placement of students with intellectual disability. Inclusion, 4(2), 56-74. doi:10.1352/2326-6988-4.2.56

Schloss, P. J., Alper, S., \& Jayne, D. (1993). Self-determination for persons with disabilities: Choice, risk, and dignity. Exceptional Children, 60(3), 215-225. https://doi.org/10.1177/001440299406000303

Schwartz, J., \& Begley, Sharon. (2003). The mind and the brain : Neuroplasticity and the power of mental force (1st pbk. ed.). New York: Regan Books/HarperCollins Publ.

Scruggs, Thomas E, \& Mastropieri, Margo A. (1996). Teacher Perceptions of Mainstreaming/Inclusion, 1958-1995: A Research Synthesis. Exceptional Children, 63(1), 59-74. https://doi.org/10.1177/001440299606300106

Silverman, J. C. (2007). Epistemological Beliefs and Attitudes Toward Inclusion in Preservice Teachers. Teacher Education and Special Education, 30(1), 42-51.

Soto, G., Müller, E., Hunt, P., \& Goetz, L. (2001). Critical issues in the inclusion of students who use augmentative and alternative communication: An educational team perspective. Augmentative and Alternative Communication, 17(2), 62-72.

Soukup, J. H., Wehmeyer, M. L., Bashinski, S. M., \& Bovaird, J. A. (2007). Classroom Variables and Access to the General Curriculum for Students with Disabilities. Exceptional Children, 74(1), 101-120. https://doi.org/10.1177/001440290707400106

Sosu, E., \& Rydzewska, E. (2017): “Are all beliefs equal?” investigating the nature and determinants of parental attitudinal beliefs towards educational inclusion, Educational Studies, DOI: 10.1080/03055698.2017.1312286

Spencer, S. (2011). Universal Design for Learning: Assistance for Teachers in Today's Inclusive Classrooms. Interdisciplinary Journal of Teaching and Learning. V.1 p1022.

Spencer-liams, J., Flosi, J. (2020) Leading for All: How to Create Truly Inclusive and Excellent Schools. Corwin Press. 
Spooner, Fred ; Waldron, Nancy L ; McLeskey, James ; Algozzine, Bob. (2014). What are the Roles of Related Service Personnel in Inclusive Schools? In Handbook of Effective Inclusive Schools (pp. 117-127). Routledge. https://doi.org/10.4324/9780203102930-14

Stanovich, P. J., \& Jordan, A. (2002). Preparing general educators to teach in inclusive classrooms: Some food for thought. The Teacher Educator, 37(3), 173-185.

State Implementation and Scaling-up of Evidence-based Practices Center (SISEP) [Online Training Modules] (2013-2018) Retrieved from: https://implementation.fpg.unc.edu/module-1/implementation-drivers

Stover, K., Kissel, B., Haag, K., \& Shoniker, R. (2011). Differentiated coaching: Fostering reflection with teachers. The Reading Teacher, 64(7), 498-509.

Storey, K. (2007). Combating ableism in schools. Preventing school failure: Alternative education for children and youth, 52(1), 56-58.

Strieker, Toni, Logan, Kent, \& Kuhel, Karen. (2012). Effects of job-embedded professional development on inclusion of students with disabilities in content area classrooms: results of a three-year study. International Journal of Inclusive Education, 16(10), 1047-1065. https://doi.org/10.1080/13603116.2010.538868

Swift Education Center. (2020). https://swiftschools.org/

Symeonidou, S., \& Phtiaka, H. (2009). Using teachers' prior knowledge, attitudes and beliefs to develop in-service teacher education courses for inclusion. Teaching and Teacher Education, 25(4), 543-550.

https://doi.org/10.1016/j.tate.2009.02.001

Tate, W. F. (2016). Chapter 4: Critical Race Theory and Education: History, Theory, and Implications. Review of Research in Education, 22(1), 195-247.

Test, D. W., Mazzotti, V. L., Mustian, A. L., Fowler, C. H., Kortering, L., \& Kohler, P. (2009). Evidence-Based Secondary Transition Predictors for Improving Postschool Outcomes for Students With Disabilities. Career Development for Exceptional Individuals, 32(3), 160-181. https://doi.org/10.1177/0885728809346960

Test, D., Fowler, C., \& Kohler, P. (2016). Evidence-Based Practices and Predictors in Secondary Transition: What We Know and What We Still Need to Know. Retrieved from: https://transitionta.org/ebp-and-predictors-in-secondarytransition/ 
Thompson, Violet F. (2012). The Relationship Between Professional Development, Efficacy and Teachers' Attitudes Toward Inclusion of Students with Disabilities. ProQuest Dissertations Publishing.

Trainor, A. A., \& Graue, E. (2014). Evaluating Rigor in Qualitative Methodology and Research Dissemination. Remedial and Special Education, 35(5), 267-274. https://doi.org/10.1177/0741932514528100

U.S. Department of Education. (2020). About IDEA. (2020??). https://sites.ed.gov/idea/about-idea/\#IDEA-History

U.S. National Council on Disability. (2018). The Segregation of Students with Disabilities. IDEA Series. Retrieved from https://ncd.gov/sites/default/files/NCD Segregation-SWD 508.pdf

Valencia, R. R. (1997). The evolution of deficit thinking : educational thought and practice. Falmer Press.

Van Laarhoven, Toni R, Munk, Dennis D, Lynch, Kathleen, Bosma, Julie, \& Rouse, Joanne. (2007). A Model for Preparing Special and General Education Preservice Teachers for Inclusive Education. Journal of Teacher Education, 58(5), 440-455. https://doi.org/10.1177/0022487107306803

Vinodrao, S. (2016). Benefits of inclusive education for students with intellectual disabled. International Journal of Education and Management Studies, 6(2), 186.

Voulgarides, C. K. (2018). Does compliance matter in special education? : IDEA and the hidden inequities of practice. Teachers College Press.

Wagner, M., Newman, Lynn, Cameto, Renee, Levine, Phyllis, Educational Resources Information Center, SRI International, \& Institute of Education Sciences. (2006). The Academic Achievement and Functional Performance of Youth with Disabilities. A Report from the National Longitudinal Transition Study-2 (NLTS2). NCSER 2006-3000. S.I.]: Distributed by ERIC Clearinghouse.

Wagner, M., Newman, Lynn, Cameto, Renee, Garza, Nicolle, Levine, Phyllis, Educational Resources Information Center, \& SRI International. (2005). After High School A First Look at the Postschool Experiences of Youth with Disabilities. A Report from the National Longitudinal Transition Study-2 (NLTS2). S.I.]: Distributed by ERIC Clearinghouse.

Waitoller, F., \& Artiles, A. (2013). A Decade of Professional Development Research for Inclusive Education: A Critical Review and Notes for a Research Program. Review 
of Educational Research, 83(3), 319-356.

https://doi.org/10.3102/0034654313483905

Watson, N., Roulstone, A., \& Thomas, C. (2012). Routledge handbook of disability studies. Routledge.

Wehman, P. (2002). A new era: Revitalizing special education for children and their families. Focus on Autism and Other Developmental Disabilities, 17(4), 194-197.

Wehmeyer, M. L. (2019). Strengths-based approaches to educating all learners with disabilities: Beyond special education. Teachers College Press.

Wells, E. (2016). The experiences of school Leaders who promote achievement among students with disabilities. (Doctoral dissertation, University of Central Florida). STARS Electronic Theses and Dissertations. https://stars.library.ucf.edu/etd/5048

White, J., \& Weiner, J. (2004). Influence of least restrictive environment and community based training on integrated employment outcomes for transitioning students with intellectual disabilities. Journal of Vocational Rehabilitation, 21, 149-156.

Wlodarczyk, K., Somma, M., Bennett, S., \& Gallagher, T. (2015). Moving toward inclusion: Inclusion coaches' reflections and discussions in supporting educators in practice. Exceptionality Education International, 25(3), 55-73.

Wolbring, G. (2008). The politics of ableism. Development (Society for International Development), 51(2), 252-258.

Yin, R. K. (2018). Case study research and applications: Design and methods (6th ed.). Sage. 


\section{Appendix A: Invitation Letter to Superintendent}

\section{Dear Superintendent,}

My name is Michael Salitore. I am a special education director in Oregon, and a doctoral student at Portland State University in the dissertation phase of the program. My research interest is understanding how to improve outcomes for students with intellectual disabilities (ID) by including them in the general education setting in meaningful ways.

The purpose of my study is to look to exemplary districts in Oregon that are improving inclusive practices, and conduct a case study to understand the leadership efforts (thinking, planning and actions) that contribute to improvement in inclusive practices for students with ID. Your district has been selected as one of three in the state who are leading inclusive placements for students with ID. The selection process began with perception data by regional special education leaders as well as county contacts from Oregon Department of Education, and affirmed by a data analysis of placement data for students with ID. With your permission I would like to include your district in my research.

The ask: I would like to interview three leaders in your district to understand from each of their perspectives how your organization went about improving inclusive practices for students with ID. The key leadership roles identified for this study are your teaching and learning director, SPED Director, and a building principal you believe is succeeding as an exemplary leader when it comes to including students with ID in the general education setting. The interviews would take about 45 minutes, to be scheduled over a video conference at their convenience. I would also like to request a review of artifacts your district has that will help add context: district continuous improvement plan, a building CIP (for the building where you recommend I speak with a principal), and any guidance documents that hold procedures for SPED placement, Teaching and Learning framework and training schedule related to inclusion of students with ID in the general education.

Following the interviews, transcription and data analysis, I will submit my initial findings to your leaders for review and respondent validation. I will also share a copy of the final write-up with yourself and your leaders. I intend to keep the name of your district and leaders confidential. I will only describe your district in terms of demographics including enrollment, numbers of students with ID, and their B5 placement data.

Please let me know if you would permit me to investigate how your organization is finding success in the realm of inclusive education for students with ID. If you decide for your organization to participate in this study, I have prepared a communication to 
stakeholders along with an informed consent form to be completed and returned. If you are interested in participating, l'd like to work with your executive assistant to make contact with the leaders you suggest. Let me know if you have any questions, comments or concerns.

Sincerely,

Michael Salitore 


\section{Appendix B: Letter to Participants}

Dear Leader,

My name is Michael Salitore. I am a special education director in Oregon, and a doctoral student at Portland State University in the dissertation phase of the program. My research interest is understanding how to improve outcomes for students with intellectual disabilities (ID) by including them in the general education setting.

The purpose of my study is to understand the leadership efforts (thinking, planning and actions) that contribute to improvement in inclusive practices for students with ID. Your district has been selected as one of three in the state who are leading inclusive practices for students with ID. The selection process began with perception data by regional special education leaders as well as county contacts from Oregon Department of Education, and affirmed by data analysis of placement data for students with ID.

I would like to invite you to take part in an interview with me to learn about incusive efforts under your leadership and from your perspective. I intend to conduct the interviews over google meet, at a time that works for you. The interview will last about 45 minutes. I would like to ask from your perspective how you went about improving inclusive practices for students with ID. Your district and yourself will remain anonymous throughout the study and in the final writeup of the study.

Following the interviews, transcription and data analysis, I will submit my initial findings to you for review and respondent validation. If you decide to participate in this study, I have prepared a consent form (attached) to be signed electronically and returned.

Sincerely,

Michael Salitore 


\section{Appendix C: Interview Protocol}

\section{Basic Information:}

Each interviewee will be asked to submit signed consent, which can be done electronically and stored in this author's google file folder on the PSU drive. Interviews will be scheduled with the interviewee and conducted by Michael Salitore. Given the remote nature of work conducted during the covid pandemic, interviews will be conducted via video conferencing, recorded and transcribed by using google meet transcription software. The recording will be titled by District code and interviewee title. The transcription will be saved and maintained in the PSU google drive under Michael Salitore's account.

\section{Introduction:}

The interviewer will need to establish rapport with the interviewee and frame the purpose of the study. Interviewer will confirm consent and answer any questions about the consent process and remind the interviewee they can stop the process at any time as consent is voluntary.

\section{Opening Questions:}

It is important to set the interviewee at ease, starting with some icebreaker questions such as how long they have been in their current role and their favorite part of their work.

\section{Interview Questions:}

Start with a review of their district data set that confirmed the district to be selected for study.

Q: What is your reaction to the data? (looking for first impressions of their perspective) Q: Have you disaggregated your placement data according to race for students with ID? Q: How do race and culture play a role in your thinking and planning related to including students with ID in the general education setting?

Q: It appears that you have been intentional in your leadership about placing students with ID in the general education setting for a significant part of their school day.

- How did your district get to this place?

Q: How is inclusive education for students with ID working from your perspective?

Q: Why is the inclusion of students with ID important to you?

$\mathrm{Q}$ : What are the organizational drivers for moving in this direction?

Q: What are the key leadership factors from your perspective that allow students with ID to learn in the general education settings?

\section{Artifact Followup:}

Q: I had a chance to read the (CIP, Framework, SPED Procedure Manual, PD schedule). Can you talk about your experience with this document and how it has supported or hindered progress toward inclusive practice?

Q: How did Race and/or culture factor into your equity priorities for improving inclusion in the general education for students with ID?

$\mathrm{Q}$ : Describe the role of educator attitudes in your district related to improving inclusion? 
Q: Did you intentionally focus on educator attitudes around inclusion? If so, describe how that became important and how you addressed it.

Q: Was ableism specifically discussed or thought about when planning to address educator attitudes?

Q: What was the planning and implementation process for inclusive strategies, from your perspective?

Q: Describe how you are supporting teachers and specialists in your efforts to improve inclusion for students with ID?

$\mathrm{Q}:$ What aspects of the educational environment are you directly leading efforts in to support inclusion for students with ID? What are those efforts and what does it look like?

Q: Tell me about your vision for continuing down this path?

Q: What would you do differently if starting over given your context?

Q: What advice would you give to someone in your position in another district or school that was interested in improving their inclusive practices for students with ID?

\section{Probes:}

Interviewer will use probes to illicit more information or to expand on an answer to a question. These may include, "tell me more", "I need more detail", or "could you explain a bit more about that."

\section{Closing:}

Interviewer will thank the interviewee for their time and ask if they have any questions. Interviewer will ask if they would be willing to participate in any follow up contact if there is anything that needs to be clarified. Interviewer will ask if the interviewee would be willing to review an interview summary to check the interviewer's perception and interpretation of the content. Close with a commitment to share the final dissertation once it is complete should they be interested. 


\title{
Appendix D: Informed Consent to Participate in Research
}

Project Title: $\quad$ Examining (Dis)Ability Segregation: A Multiple-Case Study

Exploring Leadership that Improves Inclusive Education in Oregon's K-12 Schools

\author{
Population: $\quad$ Adults, Interviews \\ Researcher: Michael Salitore, College of Education, Portland State University \\ Researcher Contact: michael.salitore@molallariv.k12.or.us (971) 678-0820
}

You are being asked to take part in a research study. The box below shows the main facts you need to know about this research for you to think about when making a decision about if you want to join in. Carefully look over the information in this form and ask questions about anything you do not understand before you make your decision.

\section{Key Information for You to Consider}

- Voluntary Consent. You are being asked to volunteer for a research study. It is up to you whether you choose to involve yourself or not. There is no penalty if you choose not to join in or decide to stop.

- Purpose. The purpose of this research is to investigate the leadership factors that allow students with intellectual disabilities the opportunity to learn in the general education setting.

- Duration. It is expected that your participation will consist of a recorded interview over video conference, possible followup conversation to clarify, then a copy of a summary of the interview to validate.

- Procedures and Activities. You will be asked to participate in one interview via videoconference, scheduled at mutually convenient time, and lasting approximately 40 minutes. You will also be invited to review a summary of your interview and provide feedback.

- Risks. Some of the foreseeable risks or discomforts of your participation include unlikely but possible professional ramifications. In the final writeup, specific districts and roles would not be identified and will remain confidential.

- Benefits. Some of the benefits that may be expected include sharing leadership practices with other leaders that produce improved conditions; insights from the findings may spur further thinking and approaches to continuous improvement; advancing the knowledge and approaches of inclusive practices in K-12 public education.

- Options. Participation is voluntary and the alternative is to not participate. 


\section{What happens to the information collected?}

Information collected for this research will be analyzed by the researcher as part of a dissertation. While this dissertation may eventually be published and will be disseminated in online research databases, all identifiable information of participating districts and participants will be kept confidential through the use of role descriptions and the exclusion of any personal identifying information.

\section{How will I and my information be protected?}

We will take measures to protect your privacy including the use of role and district descriptions in broad and general terms to hide any and all identifiable information. Despite taking steps to protect your privacy, we can never fully guarantee that your privacy will be protected.

To protect all of your personal information, all records will be stored and maintained on the PSU secure server, which is password protected. Despite these precautions, we can never fully guarantee that all your study information will not be revealed.

\section{What if I want to stop being in this research?}

You do not have to take part in this study, but if you do, you may stop at any time. You have the right to choose not to join in any study activity or completely stop your participation at any point without penalty or loss of benefits you would otherwise get. Your decision whether or not to take part in research will not affect your relationship with the researchers or Portland State University.

\section{Will it cost me money to take part in this research?}

There is no cost to taking part in this research, beyond your time.

\section{Will I be paid for taking part in this research?}

You will not receive monetary compensation for participating in this research.

\section{Who can answer my questions about this research?}

If you have questions or concerns, contact the research team at:

Michael Salitore, (971) 678-0820.

michael.salitore@molallariv.k12.or.us

\section{Who can I speak to about my rights as a research participant?}

The Portland State University Institutional Review Board ("IRB") is overseeing this research. The IRB is a group of people who review research studies to make sure the rights and welfare of the people who take part in research are protected. The Office of 
Research Integrity is the office at Portland State University that supports the IRB. If you have questions about your rights, or wish to speak with someone other than the research team, you may contact:

Office of Research Integrity

PO Box 751

Portland, OR 97207-0751

Phone: (503) 725-5484

Toll Free: 1 (877) 480-4400

Email: psuirb@pdx.edu

\section{Consent Statement}

I have had the chance to read and think about the information in this form. I have asked any questions I have, and I can make a decision about my participation. I understand that I can ask additional questions anytime while I take part in the research.

$\square \quad$ I agree to take part in this study

$\square \quad$ I do not agree to take part in this study 


\section{Appendix E: Oregon Department of Education Survey}

\section{ODE County Contact Survey 2021}

The goal of this survey is to solicit your perspective about which districts in Oregon are improving inclusive practices within the general education setting for students experiencing intellectual disability (ID). Please take a few minutes to share your perspective. Thank you!

I have had the opportunity to review the Consent to Participate in Research form sent by Michael Salitore along with the link to this form, and indicate my participation option below.

I agree to take part in this study

I do not agree to take part in this study

What counties are you assigned to as county contact? *

Short answer text

Describe efforts in your counties, by districts and schools where inclusion for students with ID in * the general education is improving.

Long answer text

In your counties, rank order your top 3 districts in your assigned counties that are providing for * or working towards providing meaningful inclusion for students experiencing ID. Please add some detail as to the basis of your selection (part of a consolidated plan for B5, conversations and planning, etc.) 


\section{Appendix F: ESD Director Survey}

\section{ESD Director Survey 2021}

The goal of this survey is to solicit your perspective about which districts in Oregon are improving inclusive practices within the general education setting for students experiencing intellectual disability (ID). Please take a few minutes to share your perspective. Thank you!

I have had the opportunity to review the Consent to Participate in Research form sent by Michael Salitore along with the link to this form, and indicate my participation option below.

I agree to take part in the study

I do not agree to take part in this study

Describe your special education leadership role at your Educational Service District? *

Short answer text

Describe efforts in your ESD's, by districts and schools where inclusion for students with ID in * the general education is improving.

Long answer text

In your ESD's, rank order your top 3 districts in your area that are providing for or working towards providing meaningful inclusion for students experiencing ID. Please add some detail as to the basis of your selection (part of a consolidated plan, conversations and planning, etc.) 